\title{
QED Experiments in Intense Fields
}

\author{
Mattias Marklund
}

Department of Physics - Umeå University - Sweden 


\title{
QED Experiments in Intense Fields
}

\author{
Mattias Marklund \\ Department of Physics \\ Umeå University \\ Sweden
}
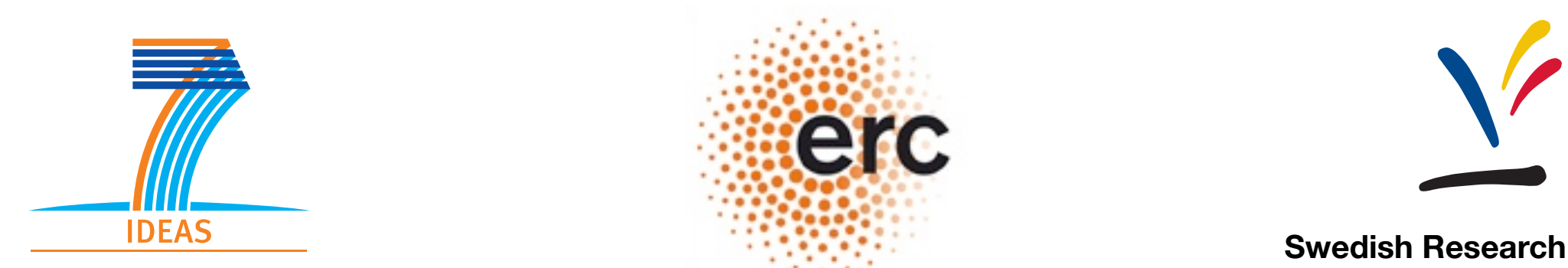

Swedish Research Council 


\section{Overview}

\section{A. Introduction}

B. Intense field generation

C. Laser development

i. Brief history

ii. Development and CPA

iii. Current and future systems

D. Secondary sources

i. Different schemes

ii. Practial and theoretical issues
E. QED and the quantum vacuum

i. Simple pictures

ii. Nonlinear effective field theory

- Heisenberg-Euler Lagrangian

- Influence on Maxwell's eqs

iii. QED effects in intense fields

- Scattering events

- Nonlinear collective effects

- Threshold phenomena 


\section{Overview}

F. Experimental tests

i. Low intensity, low energy

ii. Low intensity, high energy

iii. High intensity, low energy

iv. High intensity, high energy

v. The Unruh effect

vi. Transitions between classical and quantum

vii. Modifications of QED

\section{G. Summary}




\section{New Physics?}

- The coherent generation of massive amounts of collimated photons opens up a wide range of possibilities.

- Laboratory astrophysics, strongly coupled plasmas, photo-nuclear physics...

- Here we will focus on physics connected to the nontrivial quantum vacuum. 


\section{Background: opportunities with high-power lasers}

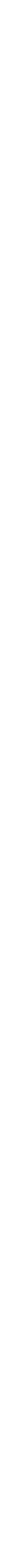

Gies, Europhys. J. D 55 (2009); Marklund \& Lundin, Europhys. J. D 55 (2009) 


\section{Background: opportunities with high-power lasers}

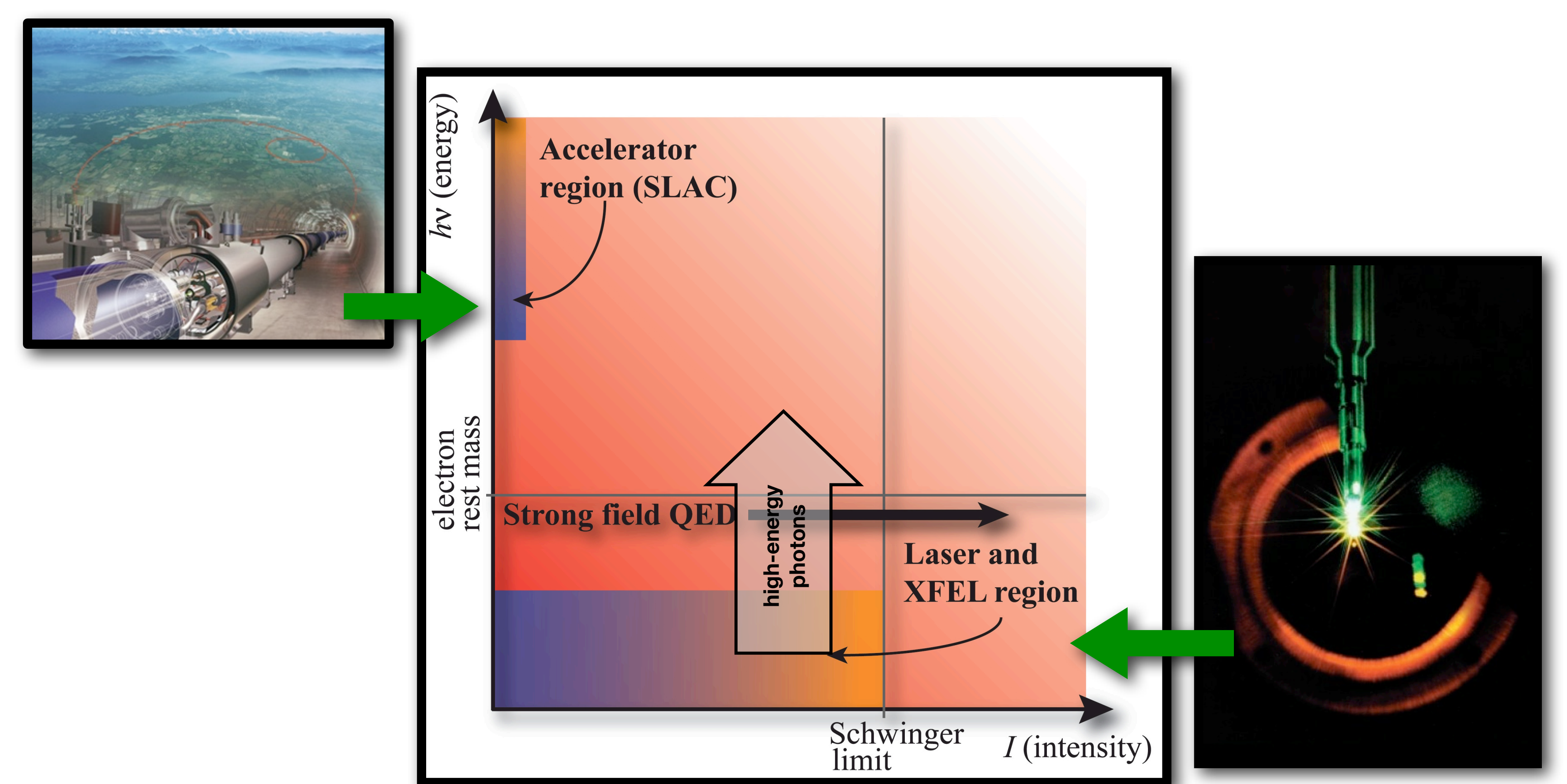

Gies, Europhys. J. D 55 (2009); Marklund \& Lundin, Europhys. J. D 55 (2009) 


\section{Laser development}

- Lasers has undergone tremendous development.

- 1917: Einstein publishes "On the quantum mechanics of radiation".

- 1958: Schawlow+Townes, light and infrared masers.

- 1959: Gould applies for "optical maser", i.e. laser, patent.

- 1960: First laser is built (Maiman and others).

- 1985: Chirped pulse amplification (Mourou and Strickland), made laser intensities above $\mathrm{GW} / \mathrm{cm}^{2}$ possible.

- 1985 - : High-field science, from atomic physics to plasmas.

- 2005: First table-top petawatt laser. 


\section{Laser development}

- Lasers has undergone tremendous development.

- 1917: Einstein publishes "On the quantum mechanics of radiation".

- 1958: Schawlow+Townes, light and infrared masers.

- 1959: Gould applies for "optical maser", i.e. laser, patent.

- 1960: First laser is built (Maiman and others).

- 1985: Chirped pulse amplification (Mourou and Strickland), made laser intensities above $\mathrm{GW} / \mathrm{cm}^{2}$ possible.

- 1985 - : High-field science, from atomic physics to
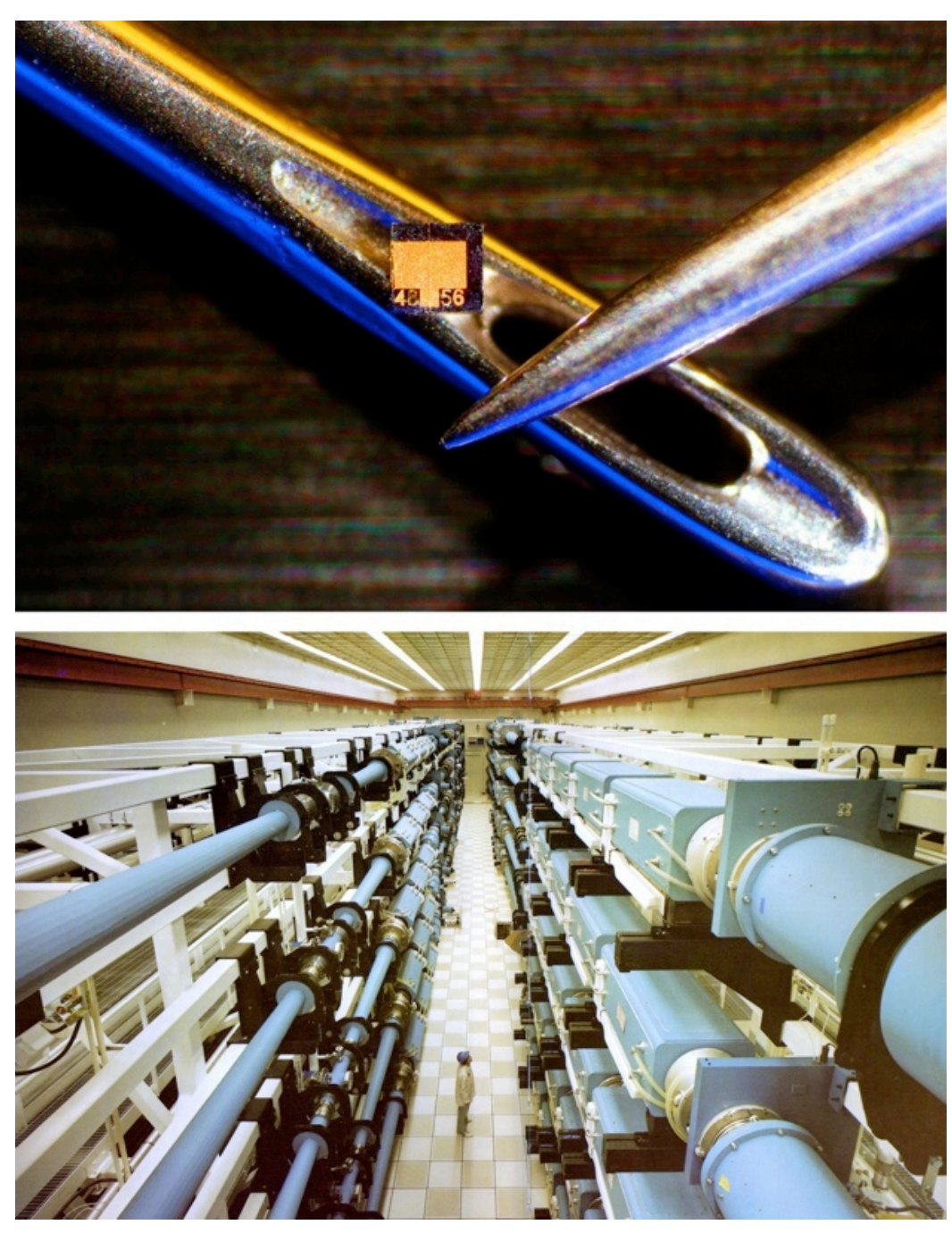
plasmas.

- 2005: First table-top petawatt laser. 


\section{Laser development}

- Lasers has undergone tremendous development.

- 1917: Einstein publishes "On the quantum mechanics of radiation".

- 1958: Schawlow+Townes, light and infrared masers.

- 1959: Gould applies for "optical maser", i.e. laser, patent.

- 1960: First laser is built (Maiman and others).

- 1985: Chirped pulse amplification (Mourou and Strickland), made laser intensities above $\mathrm{GW} / \mathrm{cm}^{2}$ possible.

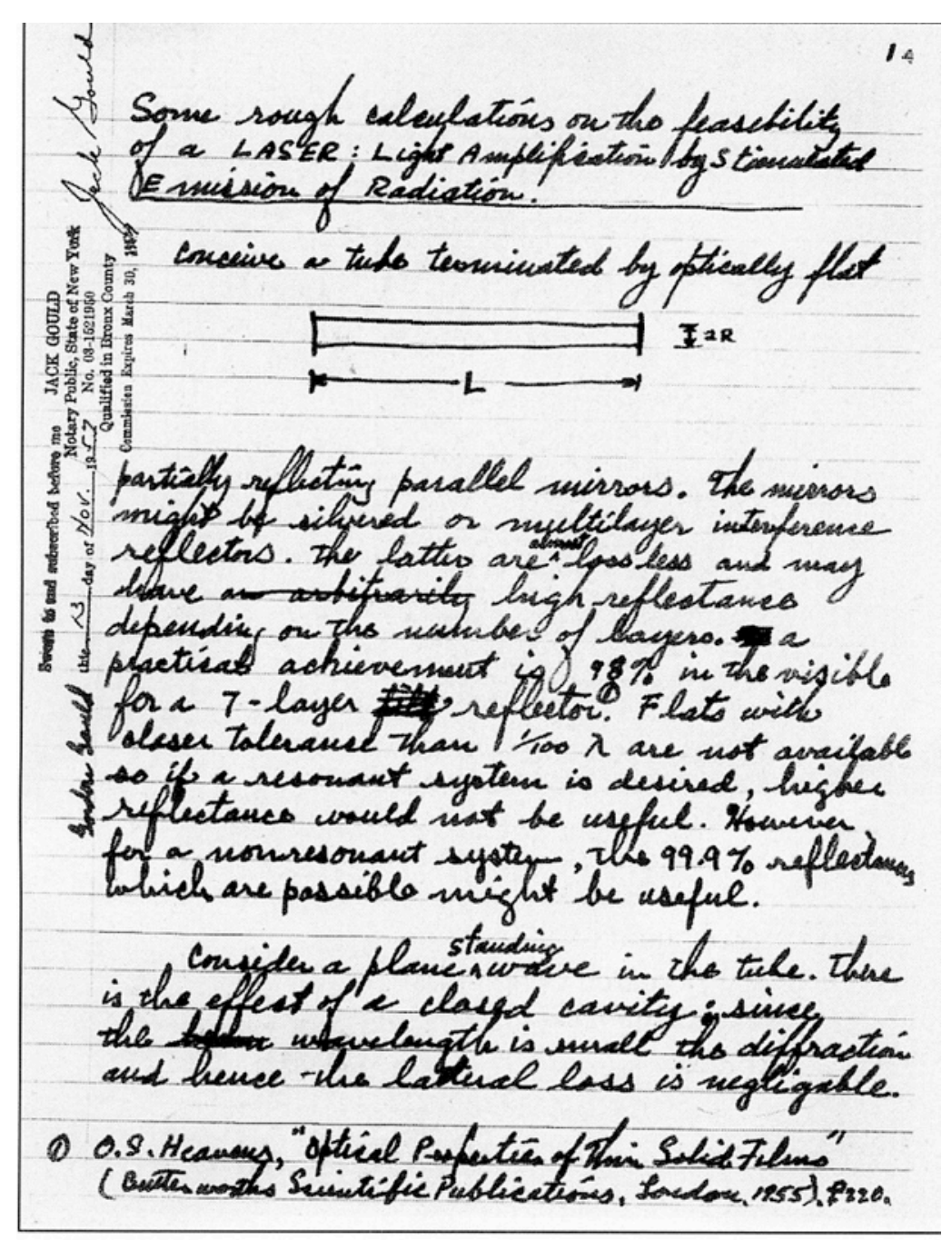

- 1985 - : High-field science, from atomic physics to plasmas.

- 2005: First table-top petawatt laser. 


\section{Laser development}

- Lasers has undergone tremendous development.

- 1917: Einstein publishes "On the quantum mechanics of radiation".

- 1958: Schawlow+Townes, light and infrared masers.

- 1959: Gould applies for "optical maser", i.e. laser, patent.

- 1960: First laser is built (Maiman and others).

- 1985: Chirped pulse amplification (Mourou and Strickland), made laser intensities above $\mathrm{GW} / \mathrm{cm}^{2}$ possible.

- 1985 - : High-field science, from atomic physics to plasmas.

- 2005: First table-top petawatt laser.

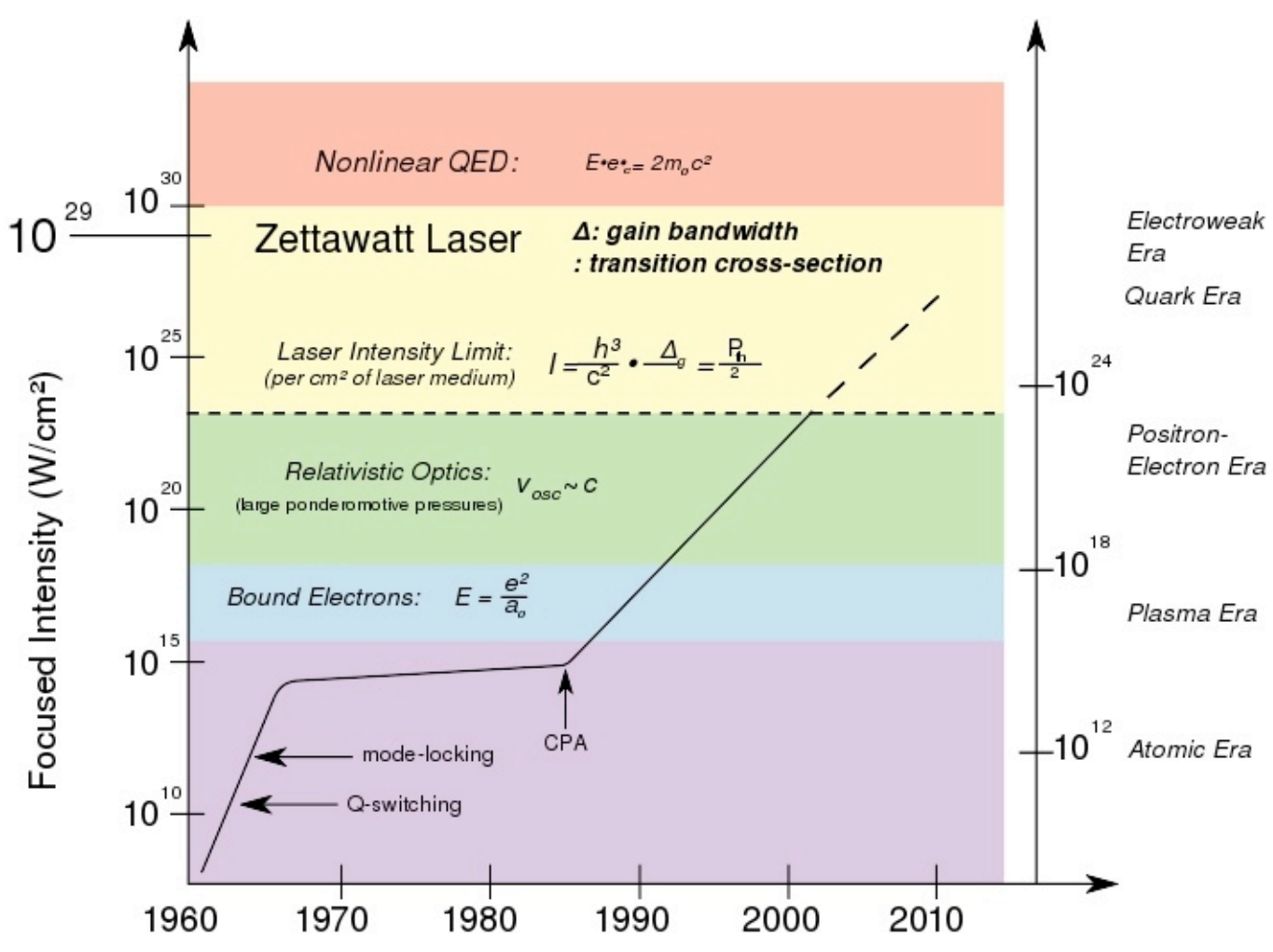




\section{Laser development}

- Lasers has undergone tremendous development.

- 1917: Einstein publishes "On the quantum mechanics of radiation".

- 1958: Schawlow+Townes, light and infrared masers.

- 1959: Gould applies for "optical maser", i.e. laser, patent.

- 1960: First laser is built (Maiman and others).

- 1985: Chirped pulse amplification (Mourou and Strickland), made laser intensities above $\mathrm{GW} / \mathrm{cm}^{2}$ possible.

- 1985 - : High-field science, from atomic physics to plasmas.

- 2005: First table-top petawatt laser.

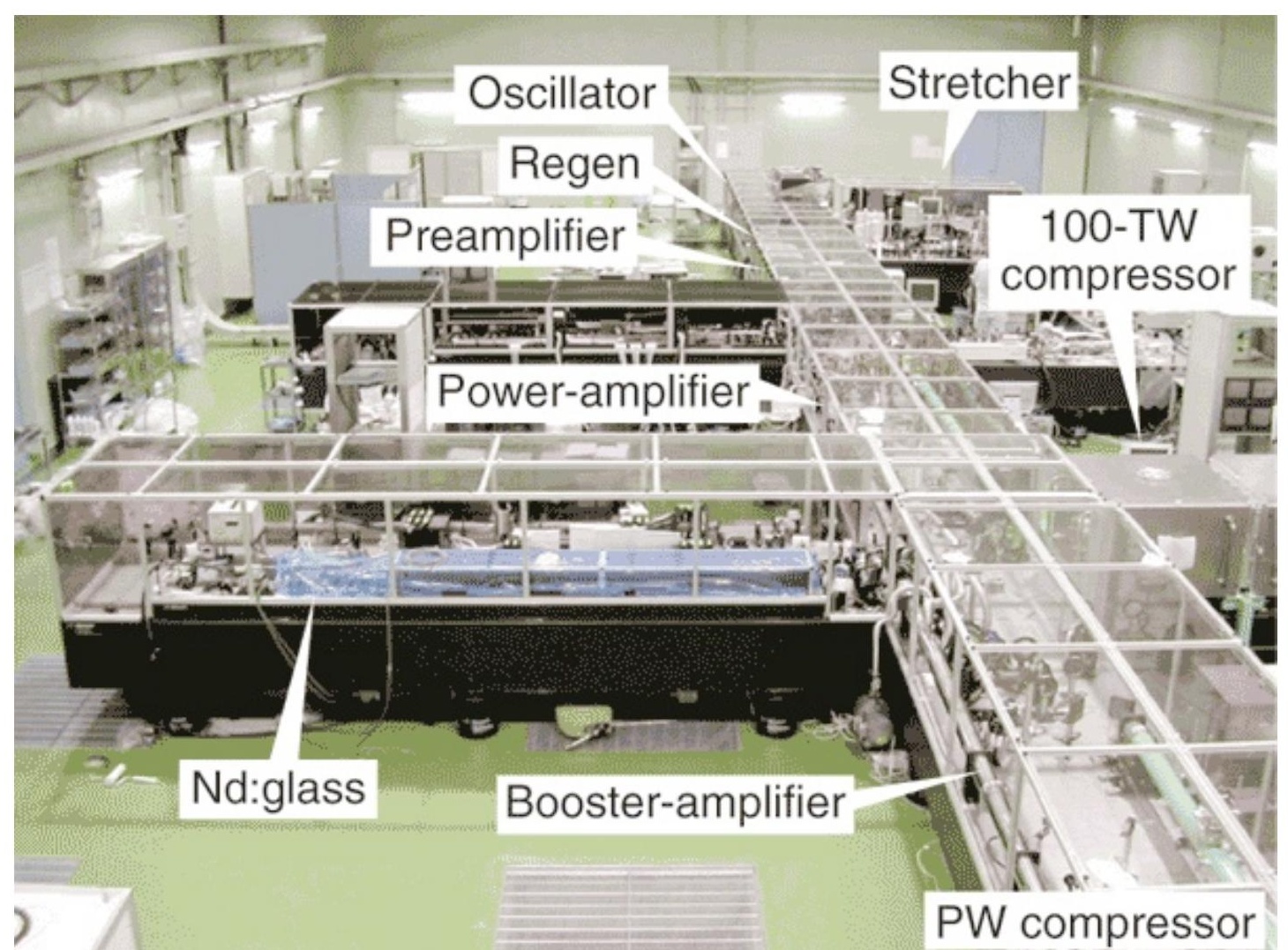




\section{Laser development}

- A tool for basic and applied science, as well as the basis for an enormous amount of applications.

- Spectral properties the key.

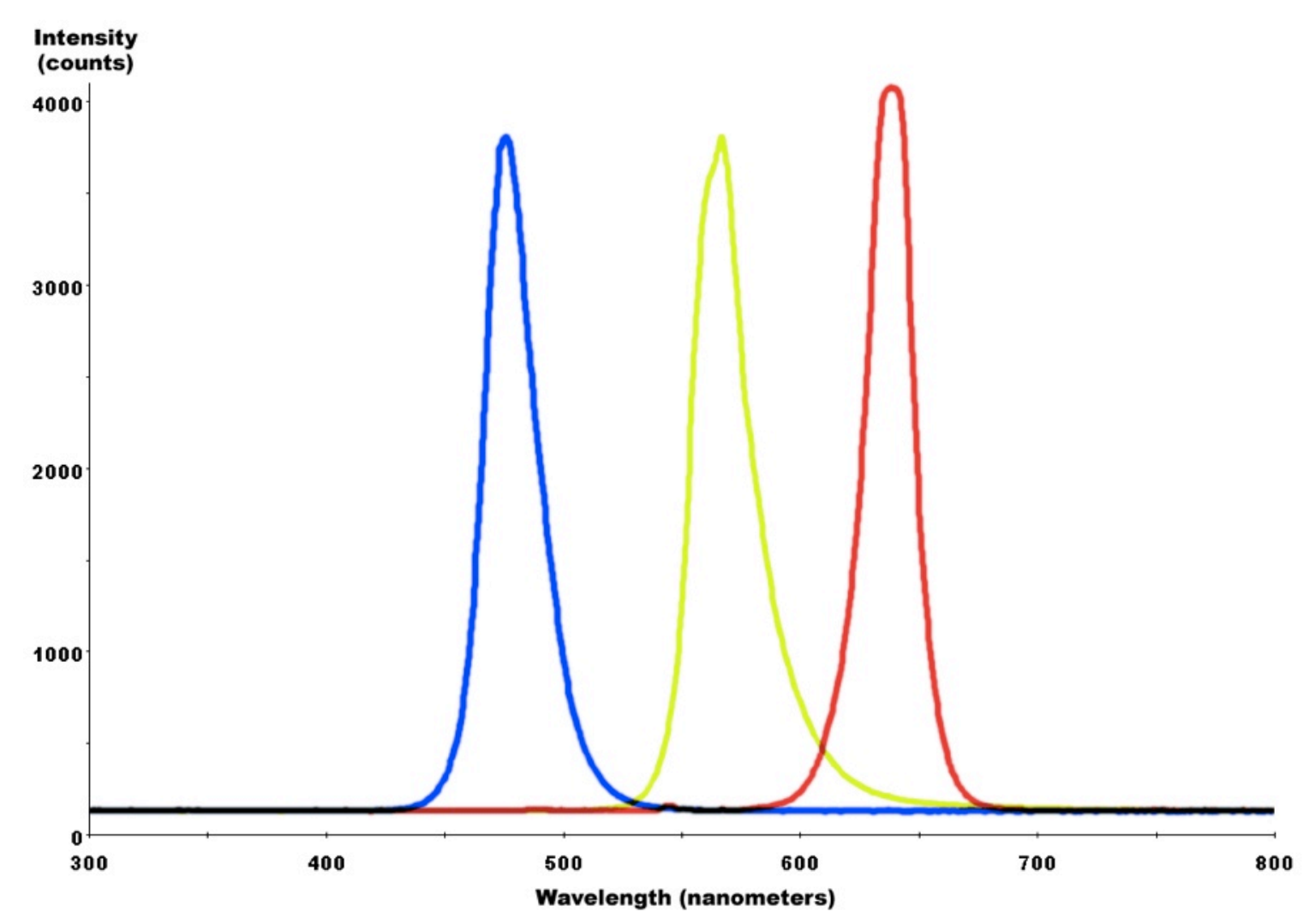

LED spectral properties

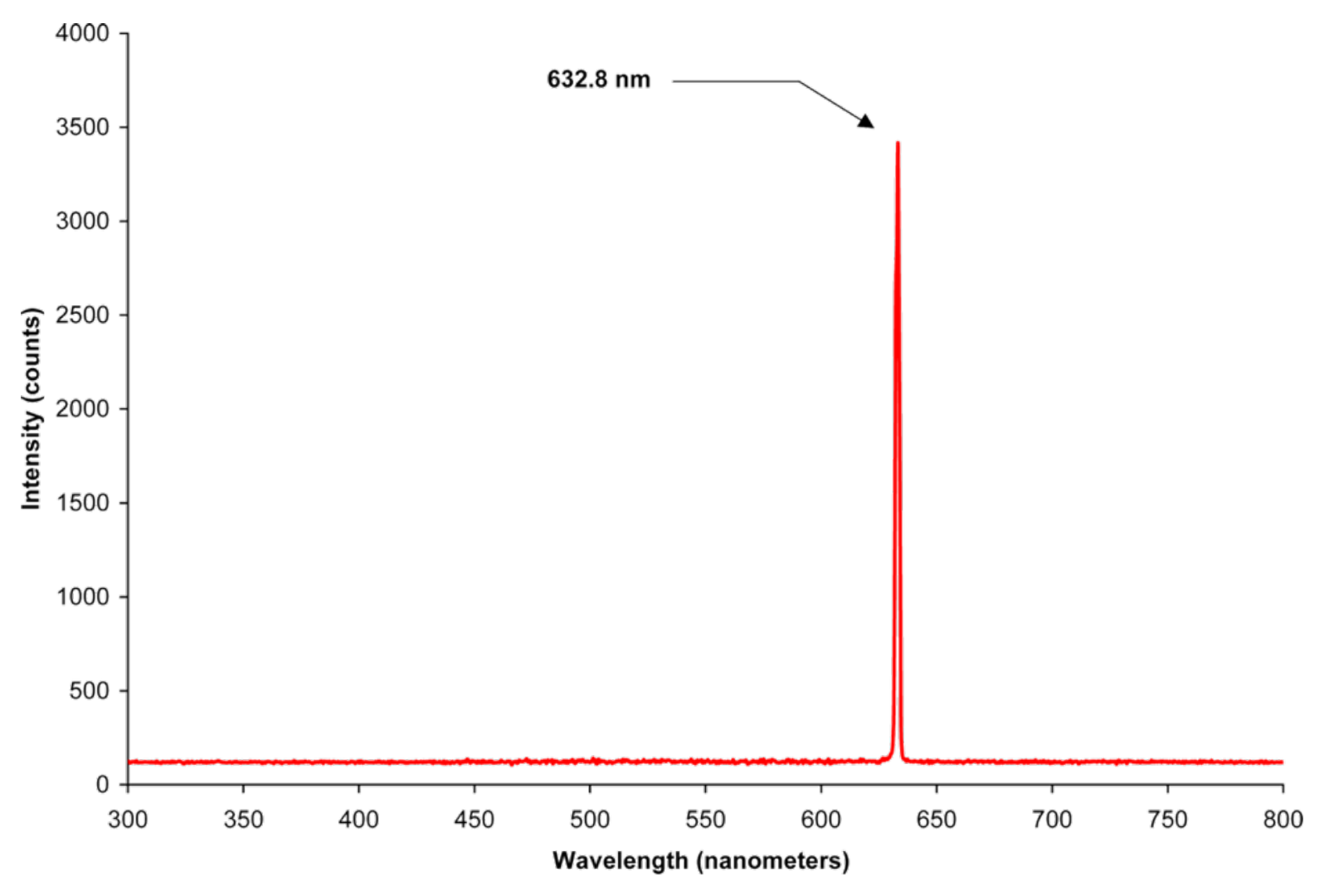

HeNe laser spectral properties 


\section{Laserlab Europe}

- A network of laser laboratories in Europe.

- Lasers laboratories at 26 places in 19 countries. Examples of high intensity laser facilities:

\begin{tabular}{|c|c|}
\hline Laboratory & Specifications \\
\hline Central Laser Facility (UK) & $\begin{array}{c}\text { Vulcan (10 PW) and Astra-Gemini (0.5 PW, multiple } \\
\text { beams, high repetition rate) }\end{array}$ \\
\hline GSI (Germany) & Combination of Phelix laser with heavy-ion accelerator \\
\hline $\begin{array}{c}\text { Instituto Superior Técnico } \\
\text { (Portugal) }\end{array}$ & $\begin{array}{c}\text { Multi-terawatt laser (e.g. electron acceleration) in } \\
\text { combination with team for large scale simulations }\end{array}$ \\
\hline Lund Laser Centre (Sweden) & $\begin{array}{c}\text { Multi-terawatt system (proton acceleration), 10 Hz } \\
\text { repetition rate (largest unit in Scandinavia) }\end{array}$ \\
\hline LULI (France) & $\begin{array}{c}\text { Two multibeam high-energy high-intensity facilities with } \\
\text { instrumentation for ICF and lab astrophysics }\end{array}$ \\
\hline
\end{tabular}




\section{High-intensity laser facilities}

- "Current" state-of-the-art laser systems

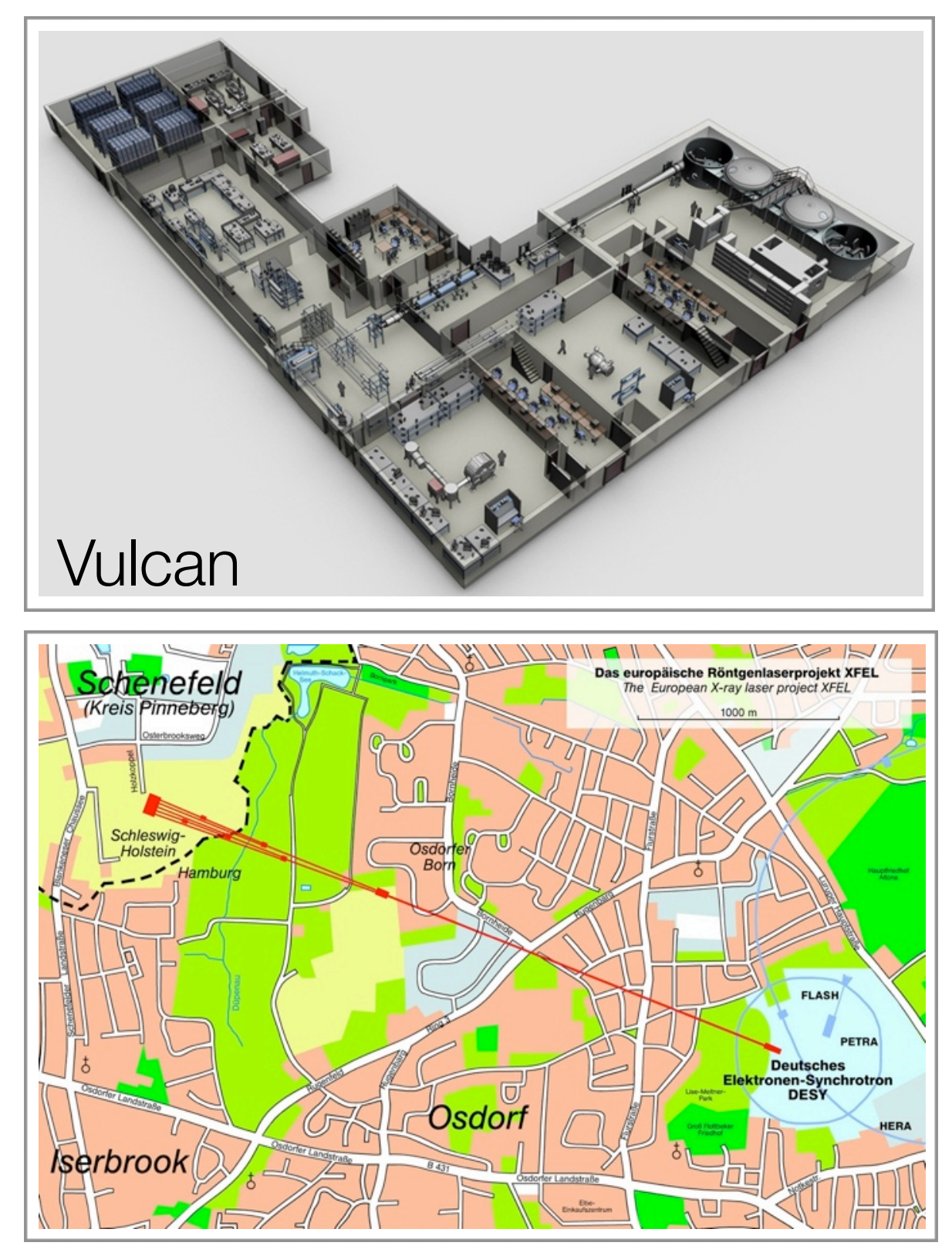

\begin{tabular}{|c|c|c|}
\hline Parameters & Vulcan (RAL) & XFEL (DESY) \\
\hline Intensity $\left(\mathrm{W} / \mathrm{cm}^{2}\right)$ & $10^{21}-10^{22}$ & $10^{14}-10^{15}$ \\
\hline Power $(\mathrm{PW})$ & 10 & $10^{-4}$ \\
\hline Pulse duration (fs) & $1-500$ & 0.04 \\
\hline Spot size $(\mu \mathrm{m})$ & $1-3$ & $\begin{array}{c}>10^{-3} \\
\text { (diffraction limit) }\end{array}$ \\
\hline Wavelength $(\mathrm{nm})$ & 800 & $<2$ \\
\hline \# of photons/shot & $10^{19}-10^{20}$ & 1018 \\
\hline $\begin{array}{c}\text { Photon energy } \\
\text { (keV) }\end{array}$ & $10^{-3}$ & 3 \\
\hline
\end{tabular}




\section{Mégajoule and NIF}

- Inertial confinement fusion facilities (Bordeaux and LLNL).

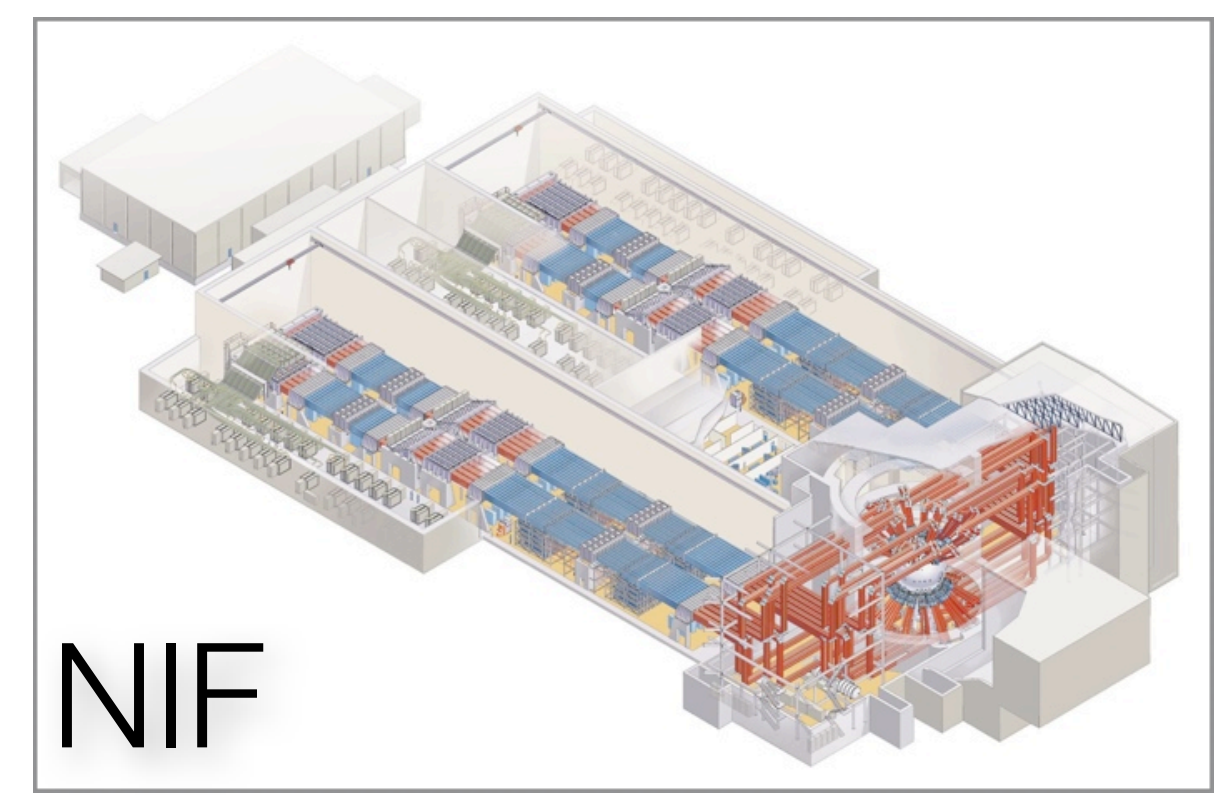

\begin{tabular}{|c|c|c|}
\hline Parameters & Mégajoule & NIF \\
\hline Power (PW) & 10 & 0.5 \\
\hline Pulse duration (ps) & $200-25000$ & few \\
\hline Wavelength (nm) & $350(\mathrm{UV})$ & $350(\mathrm{UV})$ \\
\hline \# of beamlines & 240 & 48 \\
\hline Pulse energy (MJ) & 1.8 & $4(?)$ \\
\hline
\end{tabular}

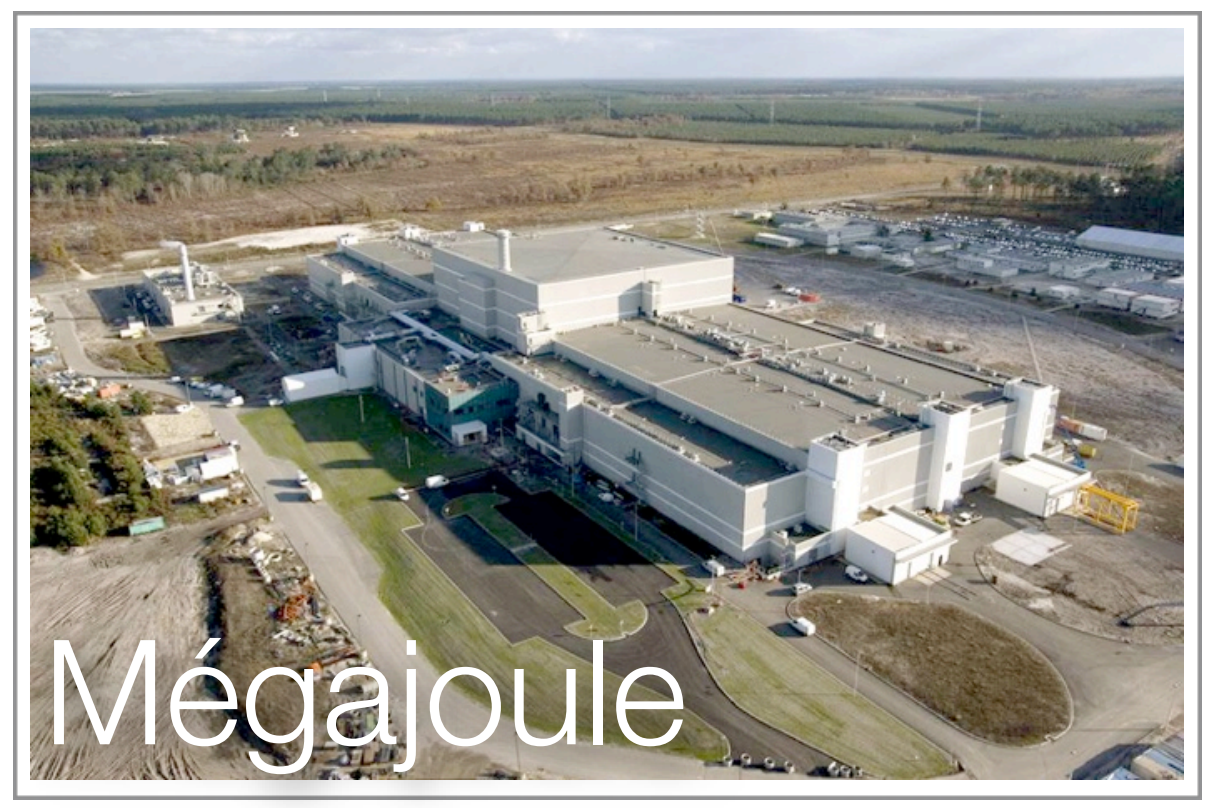




\section{High-intensity laser facilities}

- Next-generation

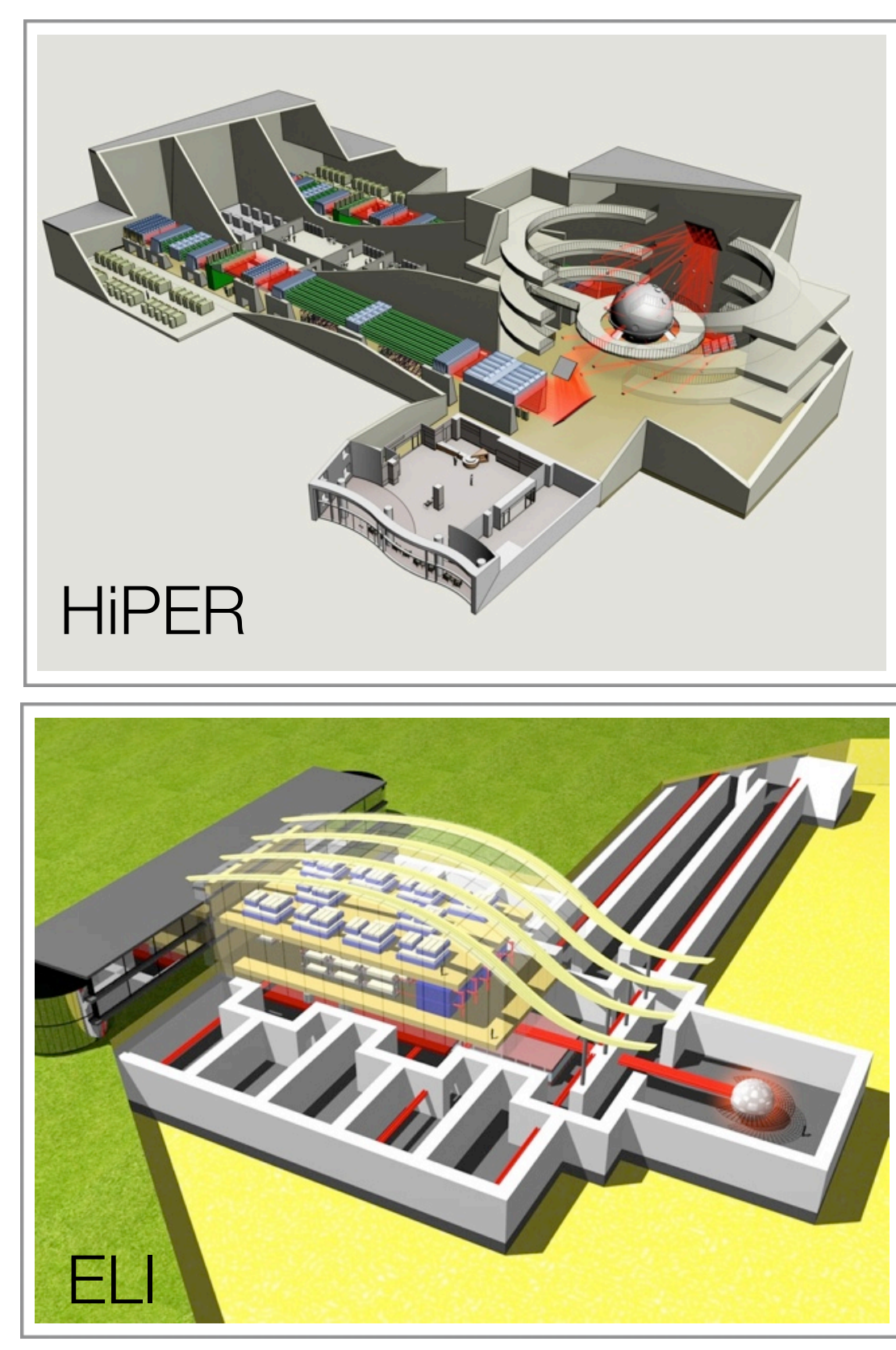

\begin{tabular}{|c|c|c|}
\hline Parameters & HPER & | \\
\hline Intensity $\left(\mathrm{W} / \mathrm{cm}^{2}\right)$ & $\begin{array}{c}10^{23}-10^{24} \\
\text { (multiple beams) }\end{array}$ & $\begin{array}{c}10^{24}-10^{25} \\
>10^{25}(\mathrm{sec} . \\
\text { sources) }\end{array}$ \\
\hline Power (PW) & 100 & 1000 \\
\hline Pulse duration (fs) & $1-5$ & 1 \\
\hline Spot size $(\mu \mathrm{m})$ & $1-3$ & $1-3$ \\
\hline Wavelength $(\mathrm{nm})$ & $\approx 800$ & $\approx 800$ \\
\hline \# of photons/shot & $10^{19}-10^{20}$ & $\approx 10^{22}$ \\
\hline $\begin{array}{c}\text { Photon energy } \\
\text { (keV) }\end{array}$ & $10^{-3}$ & $10^{-3}$ \\
\hline
\end{tabular}




\section{ELI parameters}

\begin{tabular}{|c|c|c|c|c|c|}
\hline \multicolumn{6}{|c|}{ Lasers planned for the Extreme Light Infrastructure* } \\
\hline Country & Facility focus & $\begin{array}{l}\text { Power } \\
\text { (PW) }\end{array}$ & $\begin{array}{l}\text { Pulse energy } \\
\text { (J) }\end{array}$ & $\begin{array}{l}\text { Pulse width } \\
\text { (fs) }\end{array}$ & Reprate \\
\hline Romania & $\begin{array}{l}\text { Nuclear } \\
\text { physics }\end{array}$ & $10(\times 2)$ & 200 & 20 & 0.1 \\
\hline \multirow{2}{*}{ Hungary } & \multirow{2}{*}{$\begin{array}{l}\text { Attosecond } \\
\text { physics }\end{array}$} & 1 & 5 & 5 & 1000 \\
\hline & & 20 & 400 & 20 & 0.1 \\
\hline \multirow[t]{3}{*}{$\begin{array}{l}\text { Czech } \\
\text { Republic }\end{array}$} & \multirow{3}{*}{$\begin{array}{l}\text { Secondary } \\
\text { beam } \\
\text { radiation, } \\
\text { high-energy } \\
\text { particles }\end{array}$} & 1 & 10 & 10 & 10 \\
\hline & & 5 & 50 & 10 & 10 \\
\hline & & $10(\times 2)$ & 200 & 20 & 0.1 \\
\hline $\begin{array}{l}\text { To be } \\
\text { determined }\end{array}$ & $\begin{array}{c}\text { High } \\
\text { intensity }\end{array}$ & \multicolumn{4}{|c|}{$\begin{array}{l}10 \text { beams of } 10-20 \mathrm{PW} \text { each, phased and combined to } \\
\text { create total power of } 100-200 \mathrm{PW}\end{array}$} \\
\hline
\end{tabular}

From Feder (2010) 


\section{Magnetic field generation}

- Laboratory fields from a few tesla to 1000 tesla, depending on time scale (see talk by Rikken). Relatively large scale.

- Relativistic laser-plasma interactions can create fields of the order gigagauss, on micrometer scales, stationary on pikosecond scales. Relative laser pulse of femtosecond, this is a long time.

- Heavy ion collosions, RHIC, produce chiral fields, 100000000000000000 gauss. However, on QCD scales, both in time ans space.

- Strong fields for lab astrophysics, search for WIMPs (see talk by Jaeckel), and wakefield acceleration. 


\section{Science on high-intensity lasers}

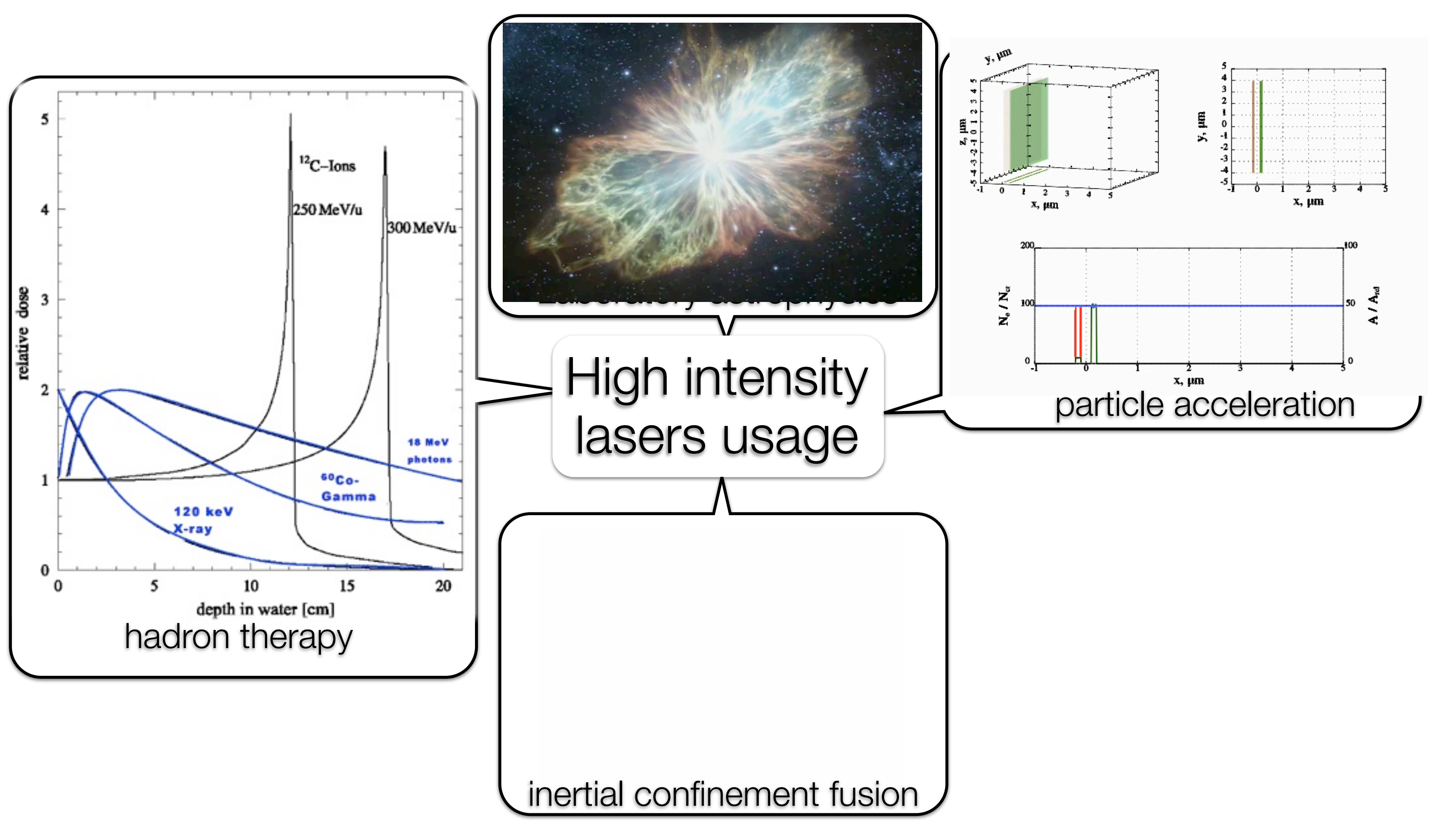




\section{Inertial confinement fusion}

- Magnetic confinement fusion in toka/spheromaks or stellarators. Inherent problem with instabilities; improved running with scaling.

- Lawson criteria: fusion heating exceeding losses

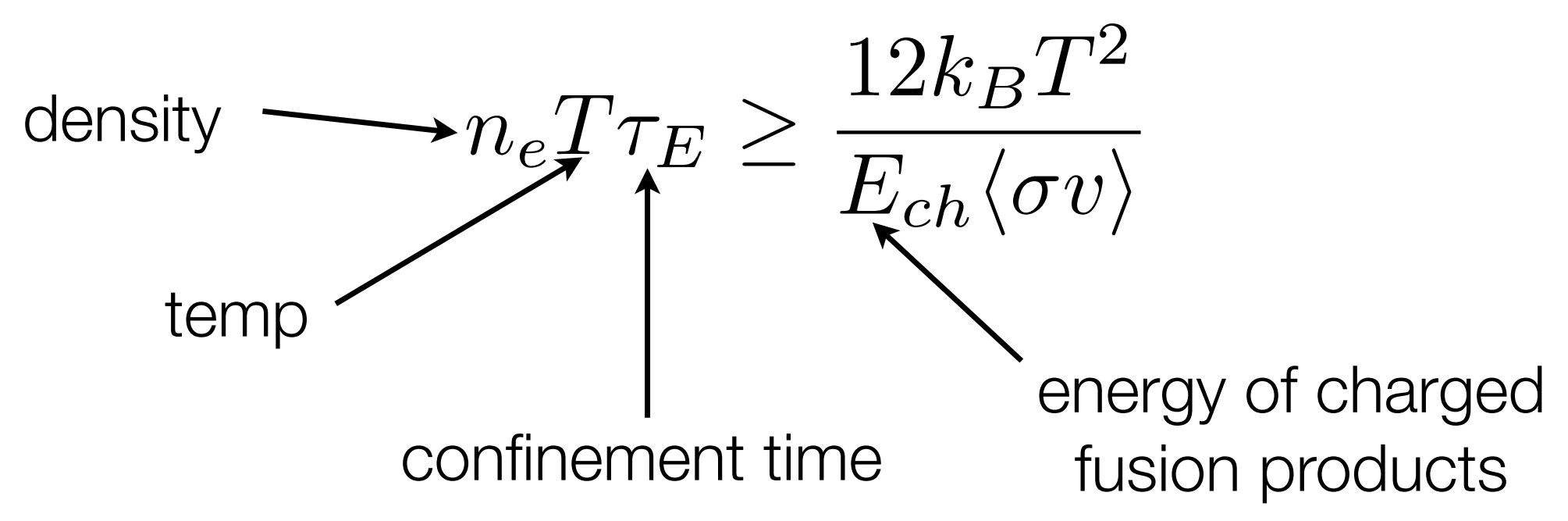

- For inertial confinement fusion, this translates into a relation between mass density and radius. $10^{3}-10^{4}$ times solid D-T density. 


\section{Inertial confinement fusion}

- Mechanisms of ICF
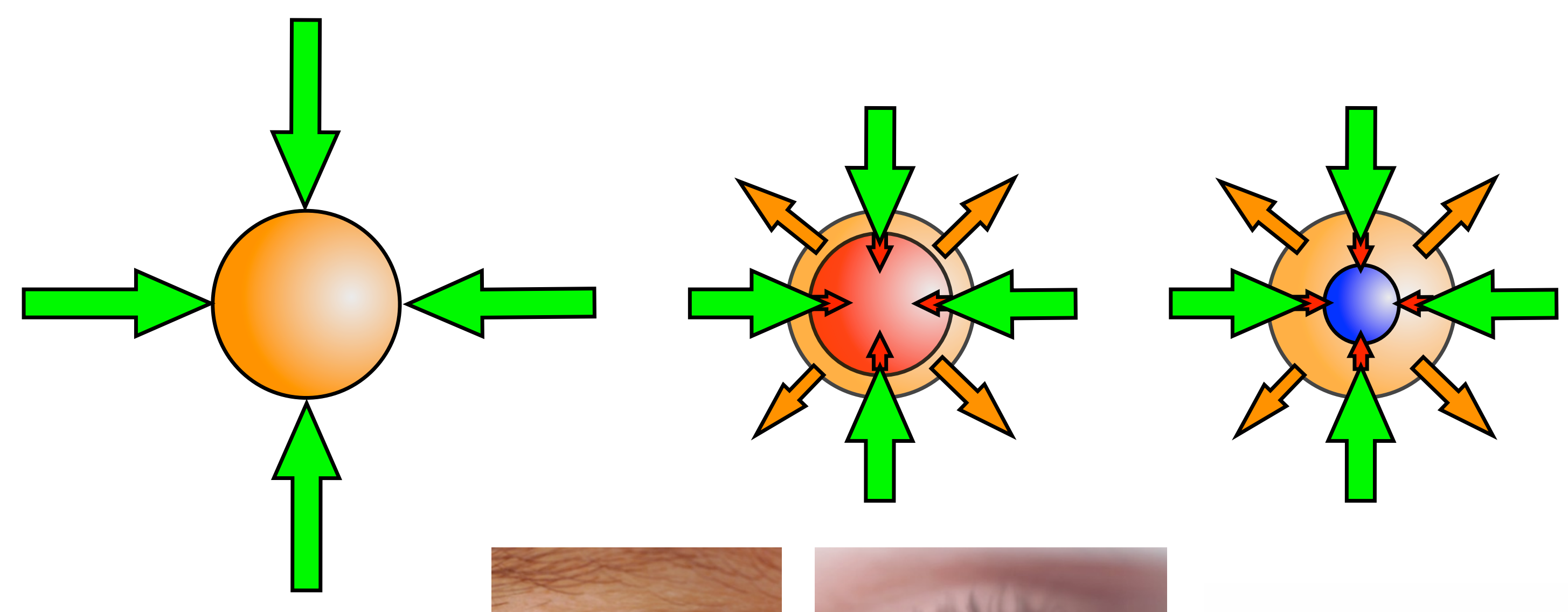

- Different drives.

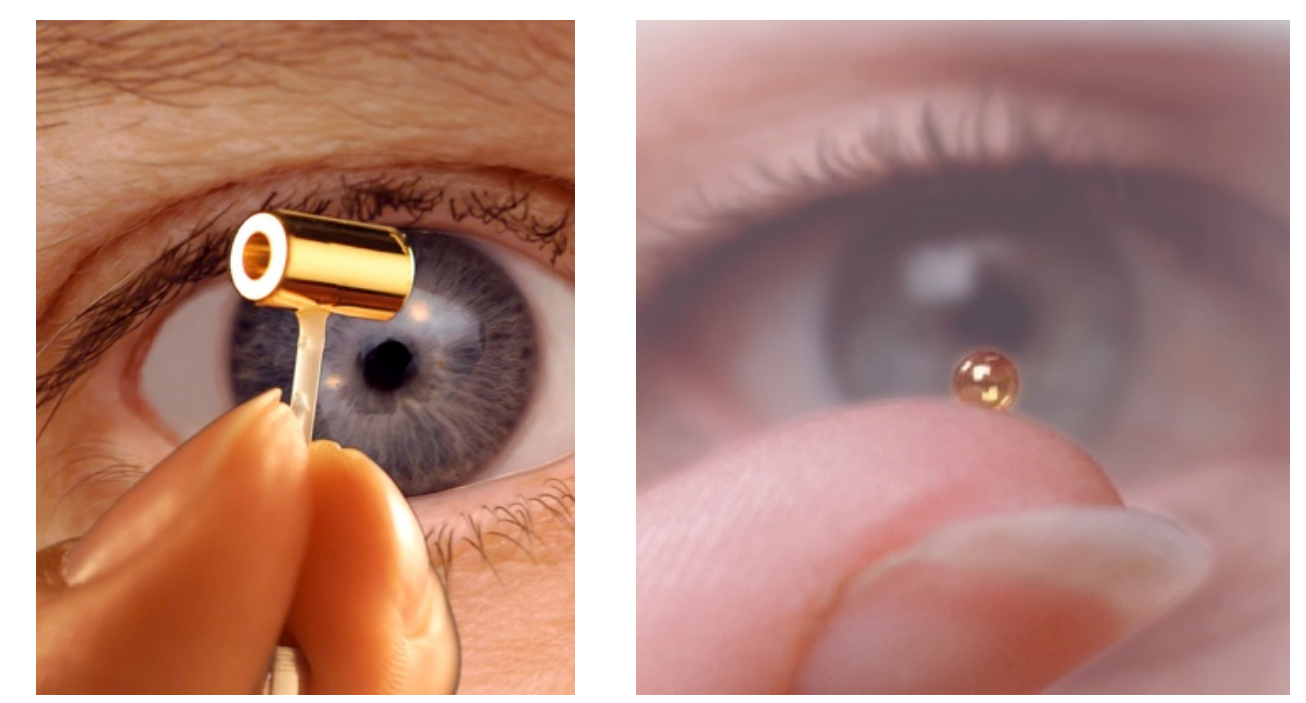




\section{Laboratory astrophysics}

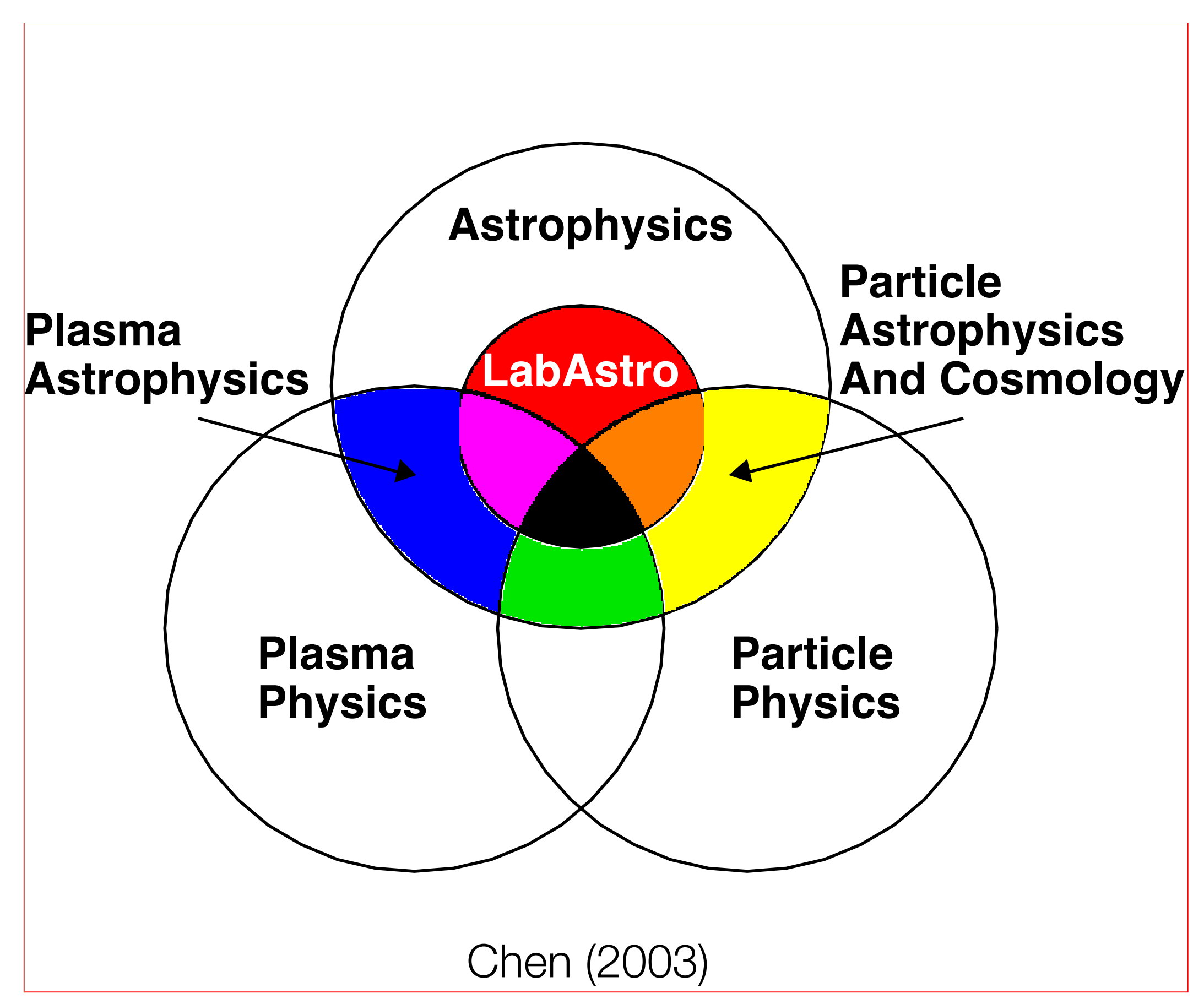




\section{Laboratory astrophysics}

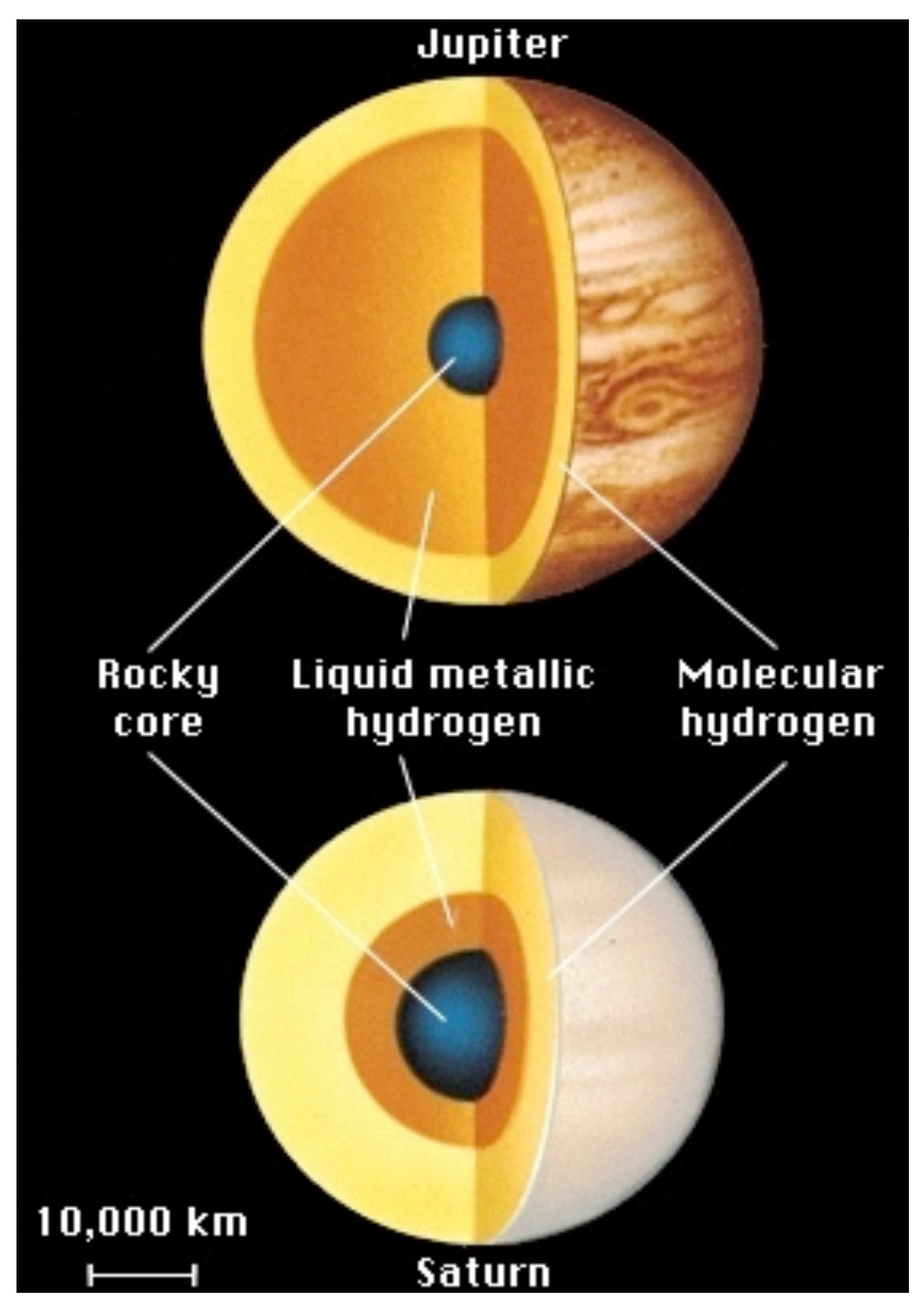

- Planetary interiors and extreme equations of state.

- Astrophysical turbulence.

- Hypersonic or relativistic jets.

- Shock wave formation and dynamics.

- Acceleration studies in plasma environments. 


\section{Particle acceleration}

-Do we need laser based accelerators?

- Compact alternative to standard accelerator schemes?

- Hadron therapy units with high patient flow.

- Cheaper (<100 M€) per unit.
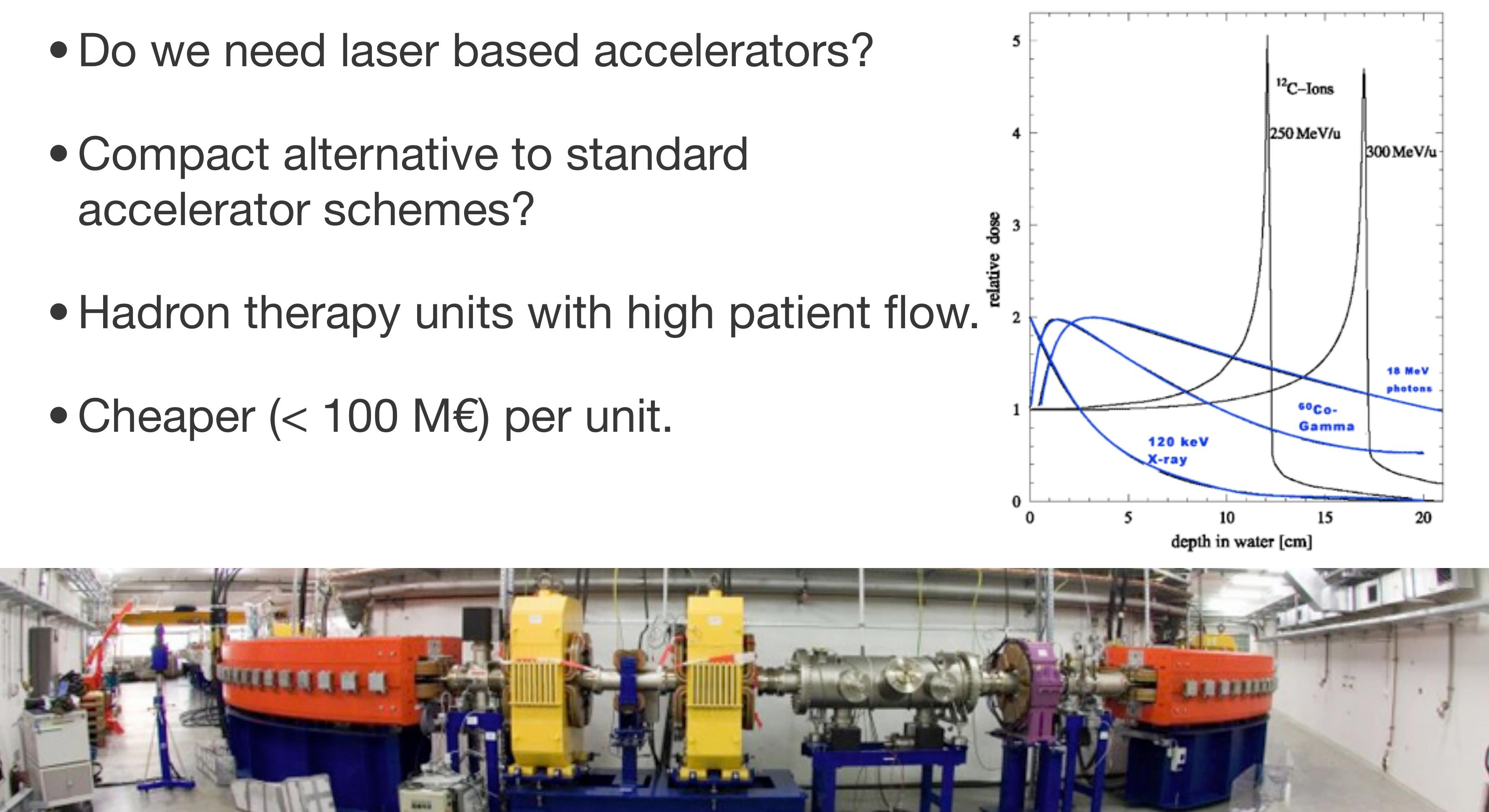


\section{Hadron acceleration}

- Procedure: let high intensity laser hit thin metal foil with "dirt".

- Problem: need monochromatic highly collimated beam.

- Brute force: spread of energies as well as spatial distributions.

- Need new geometries. Can use strong surface fields set up by laser impinging on foil.
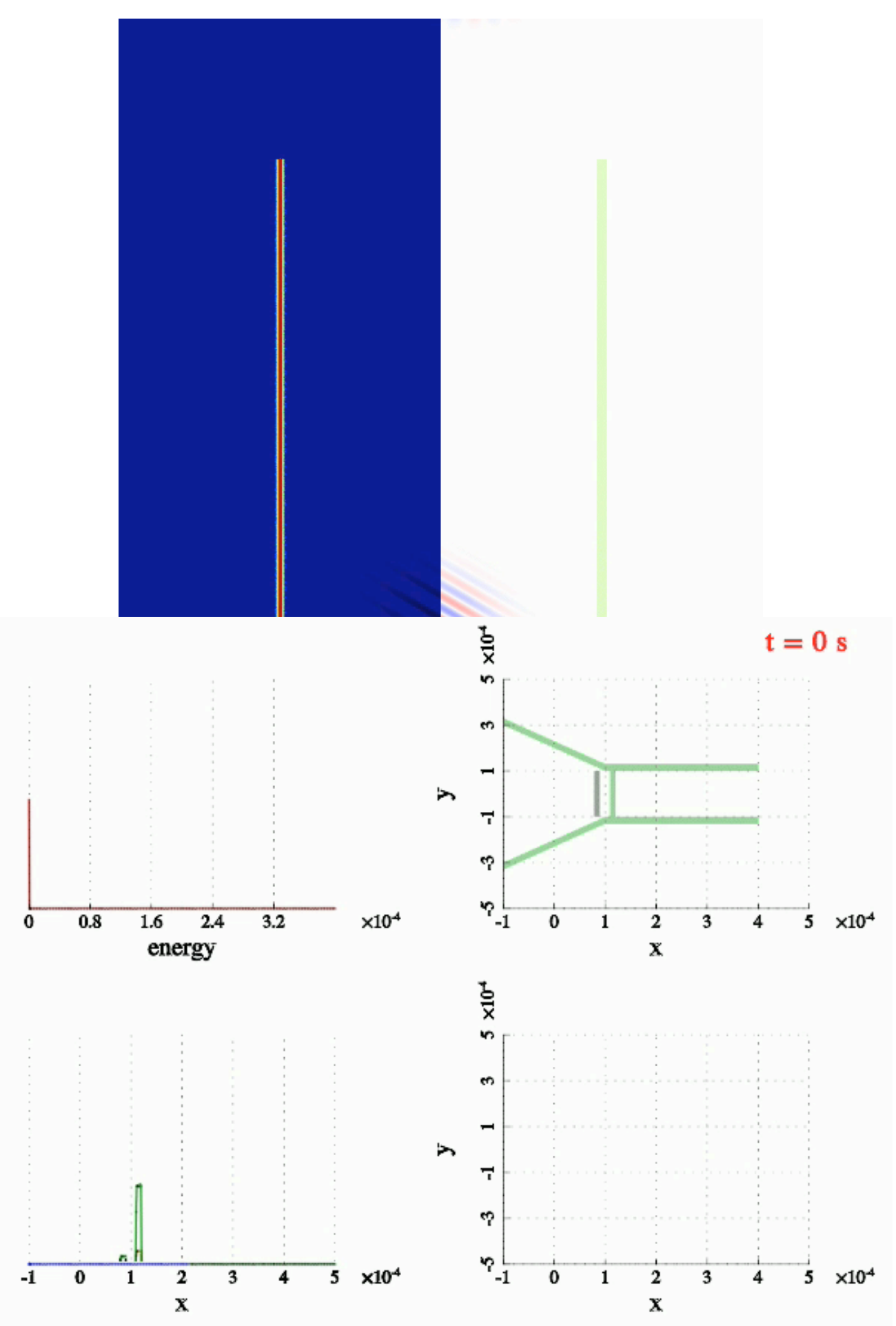

- Relativistic self-induced transparency. 


\section{Strong field generation}

- Many high phenomena directly tractable with next generation laser systems.

- Some phenomena require intensities above current experimental limits.

- Attosecond electron bunches creates mirror (Naumova et al., PRL, 2004; Nees et al., J. Mod. Opt., 2005; Thaury et al., Nature Phys., 2007).

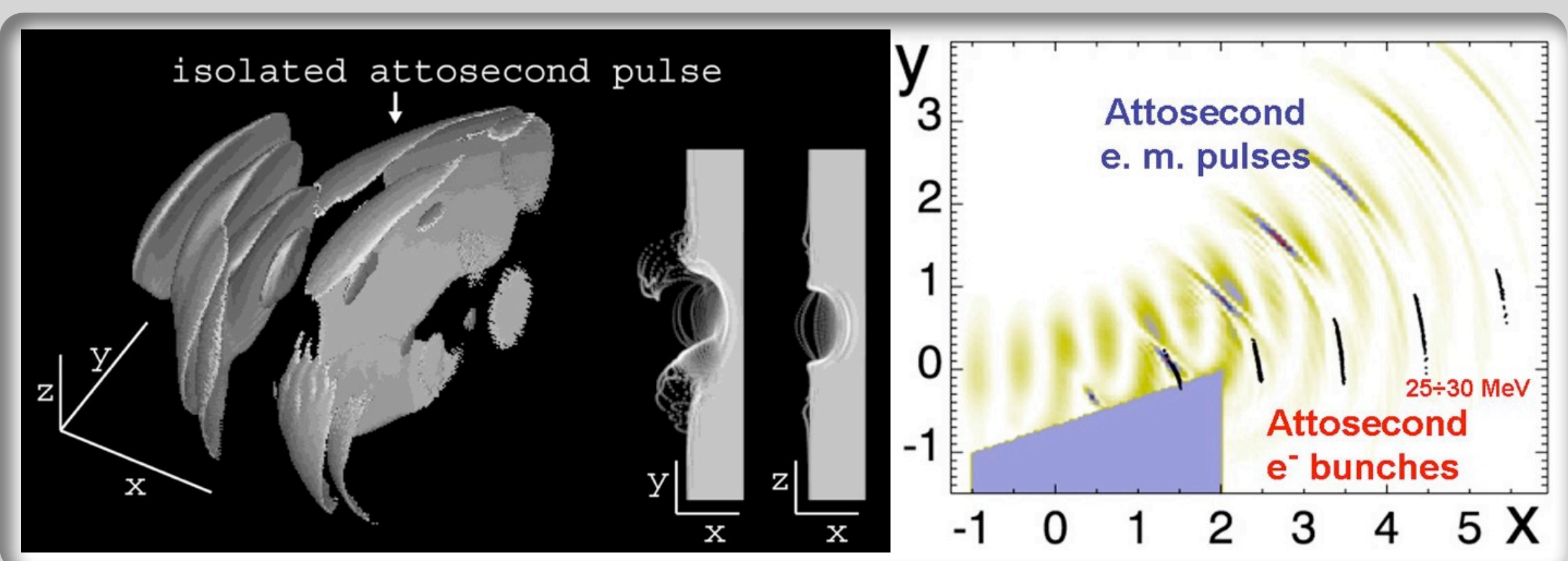




\section{Strong field generation}

Some recent simulations on attosecond pulse generation and amplification (Gonoskov et al. 2011) (a)

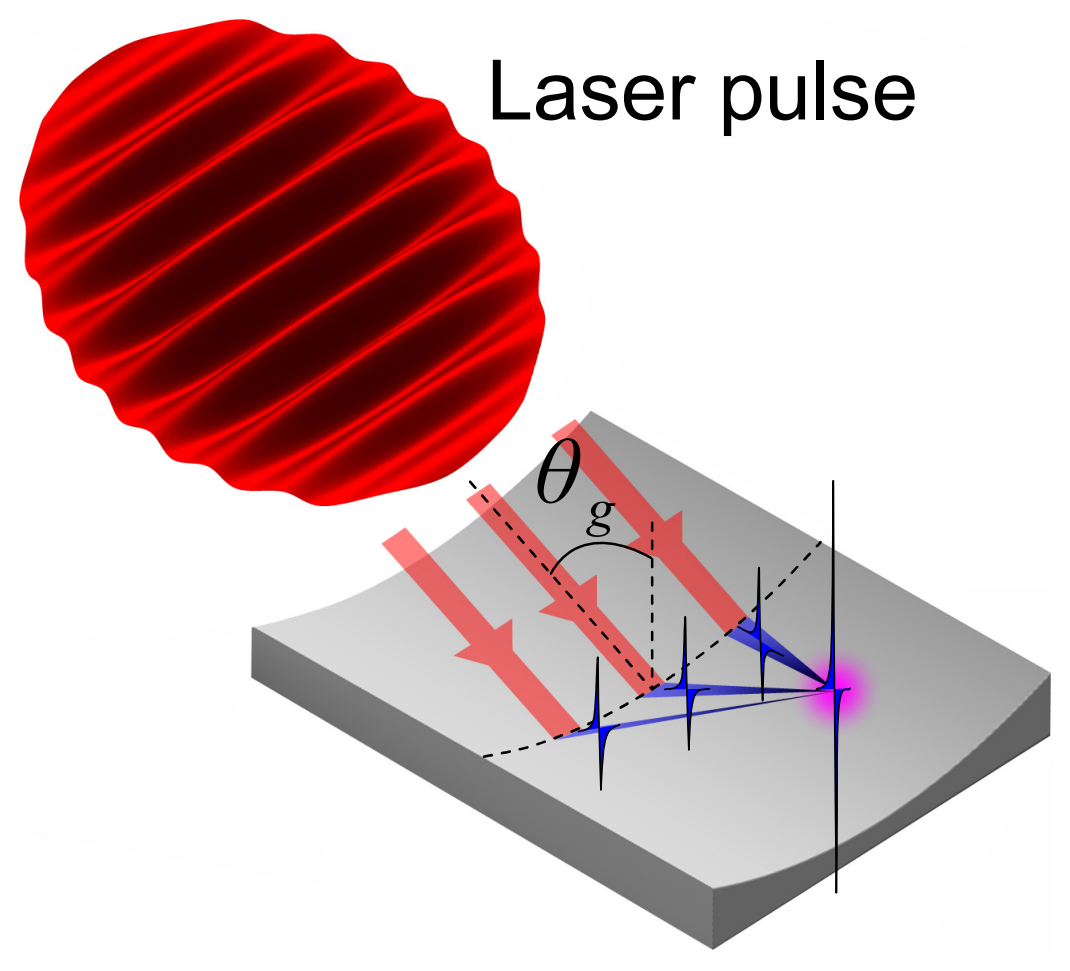




\section{Strong field generation}

Some recent simulations on attosecond pulse generation and amplification (Gonoskov et al. 2011)

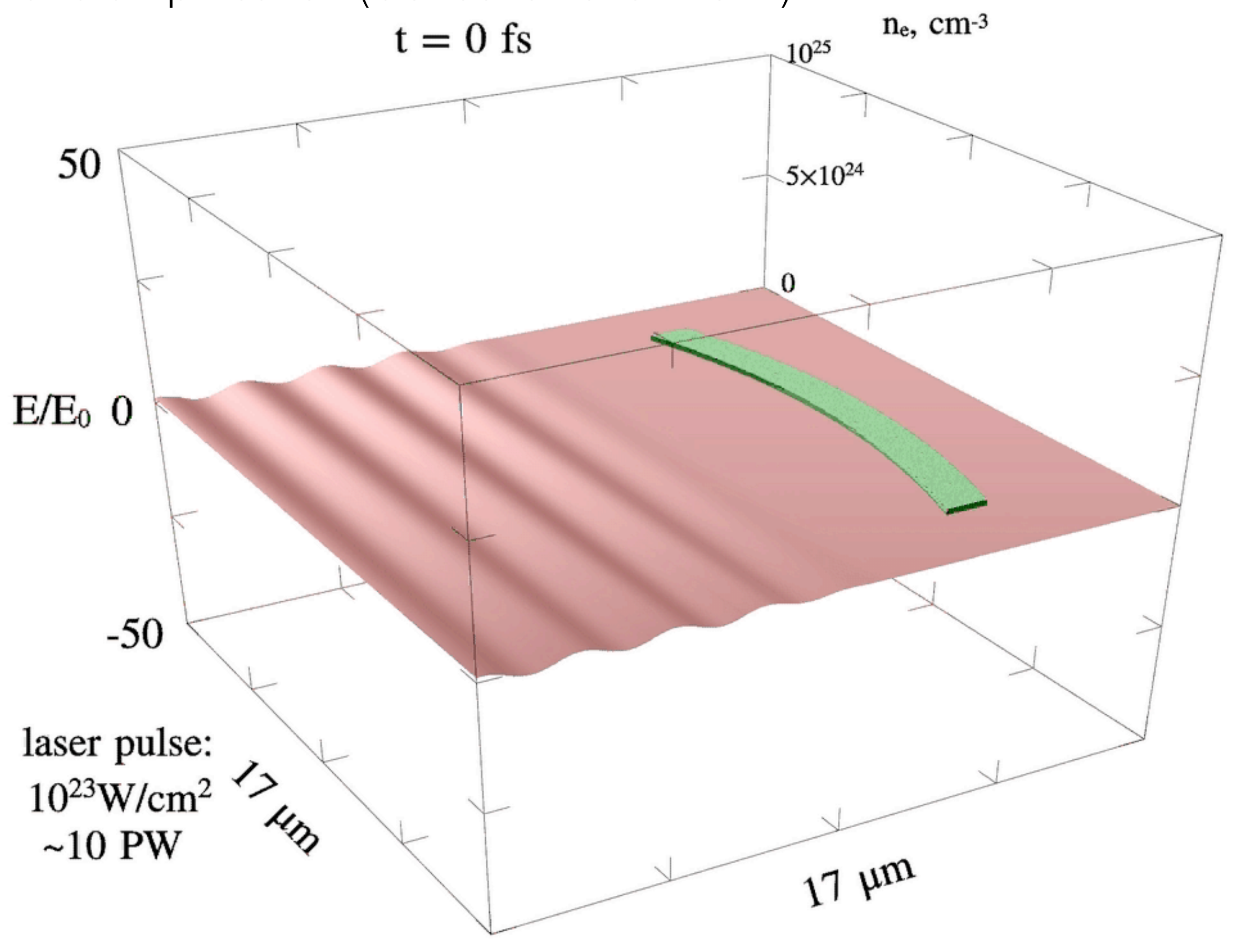

(a)

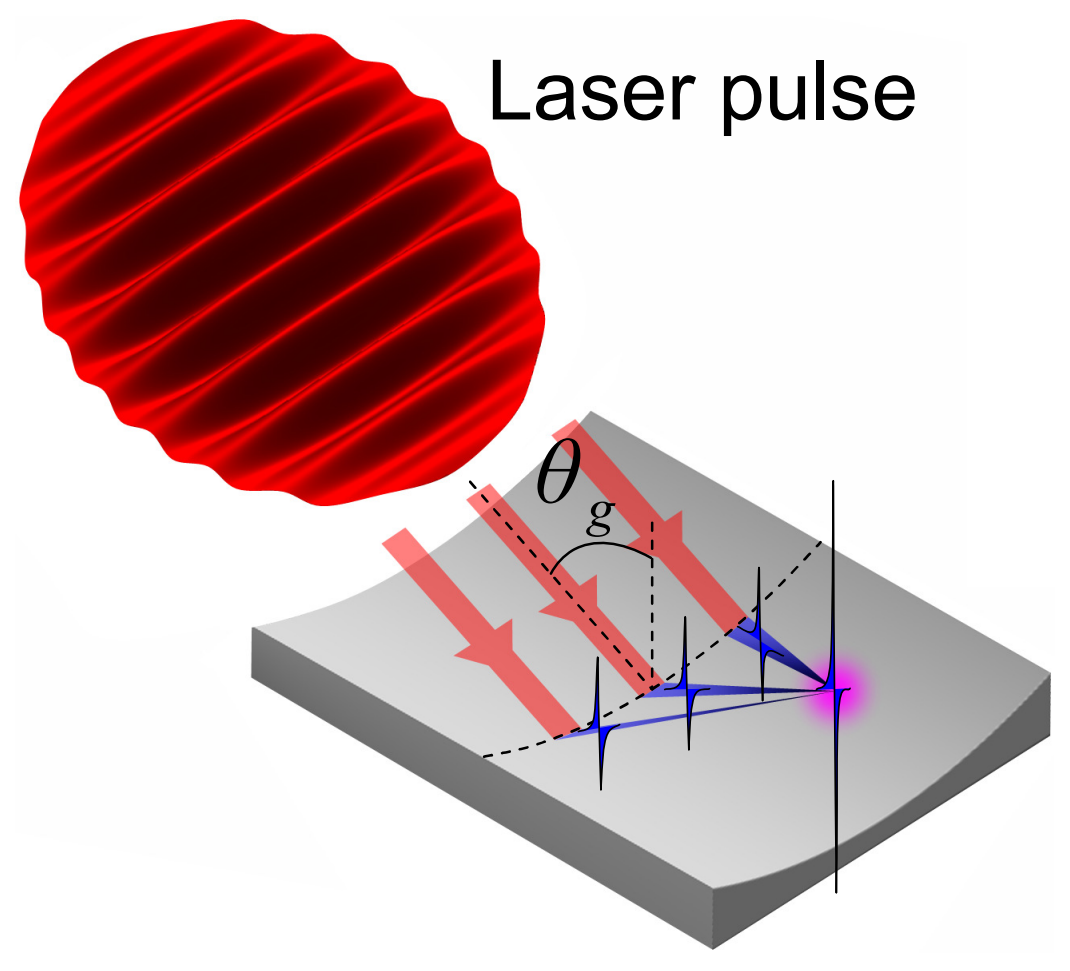




\section{Probing new regimes}

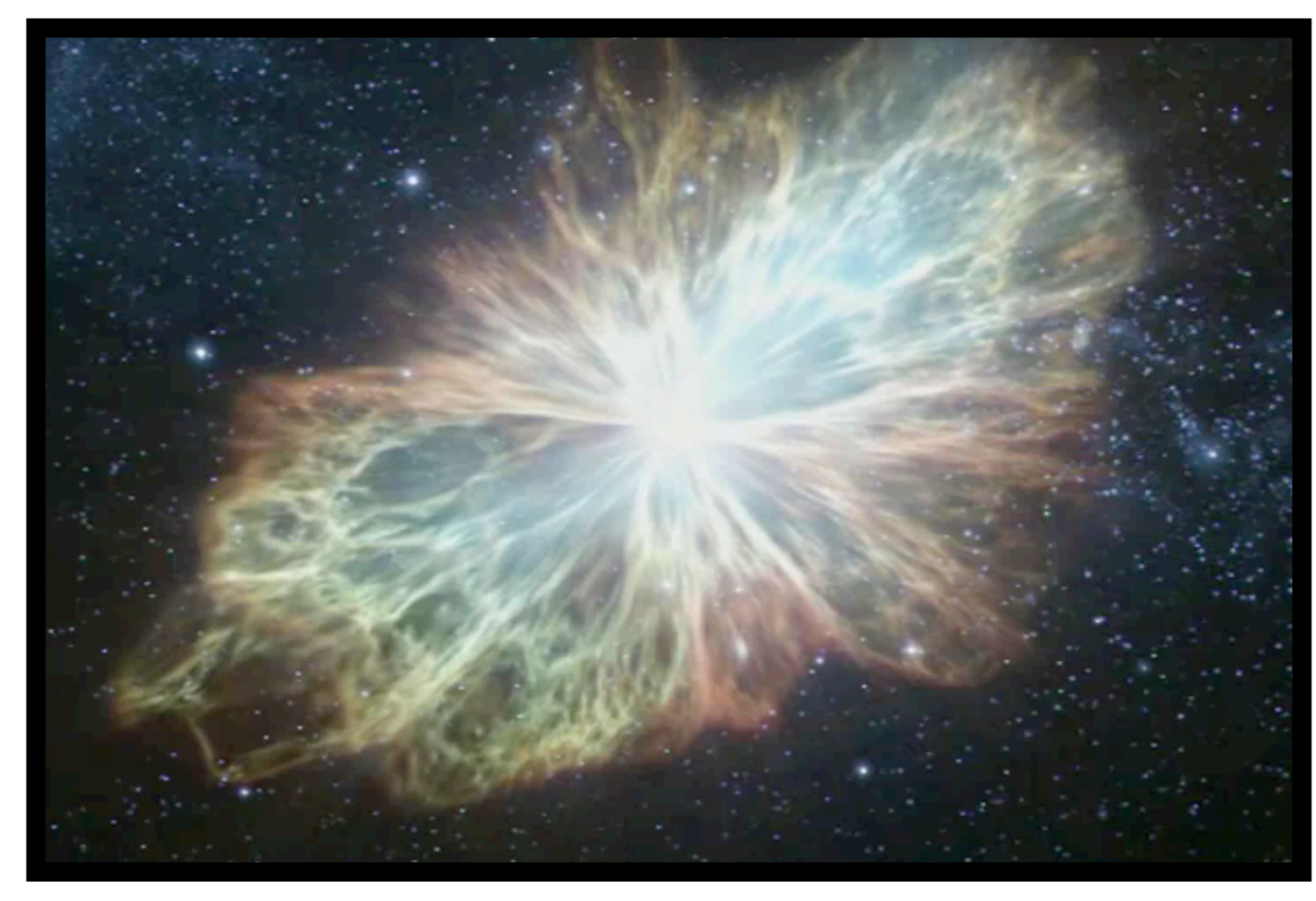

Astrophysics

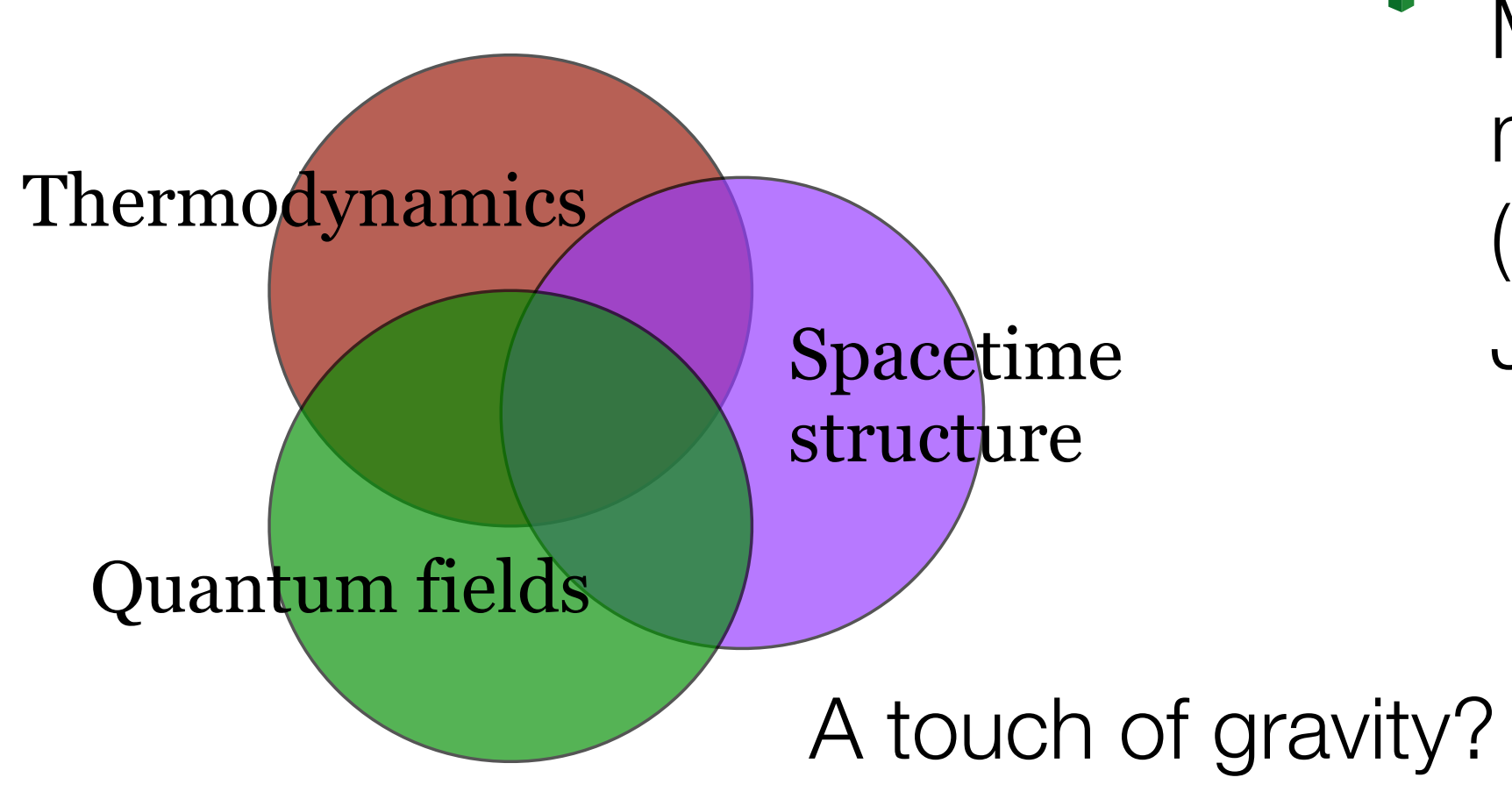

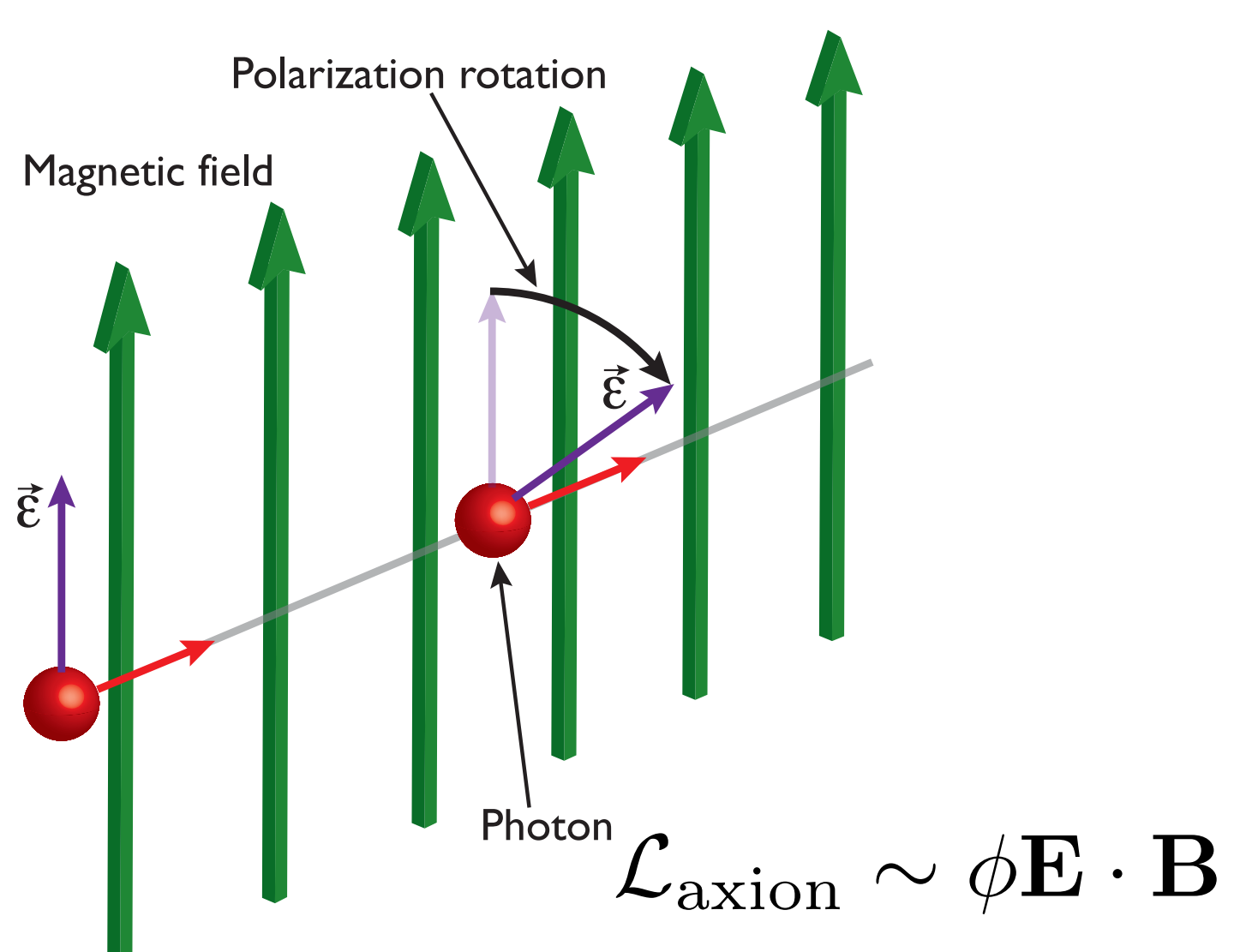

Modifications standard model, e.g. axions ("shining light through walls", Jaeckel, Amiranoff et al.) 


\section{Multi-photon processes in intense fields}

- Nonlinear Compton scattering

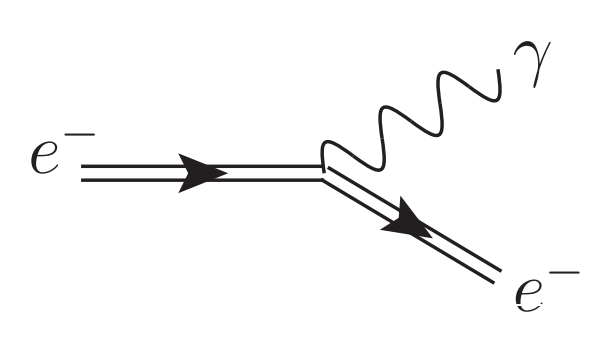

- Stimulated pair production

- Birefringence

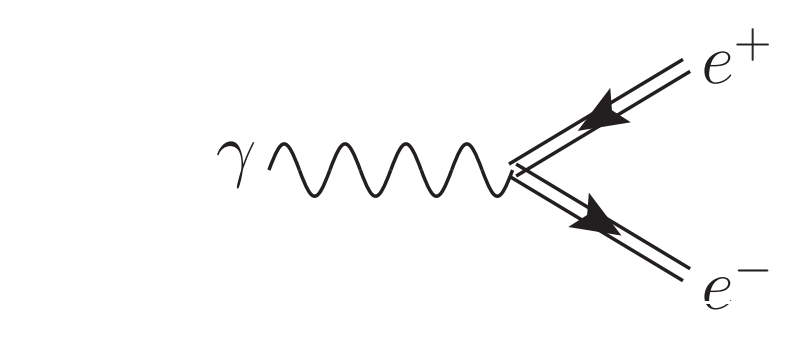

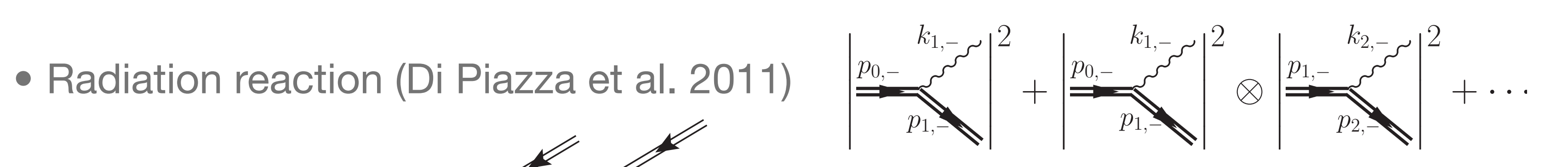

- Cascading

- Trident
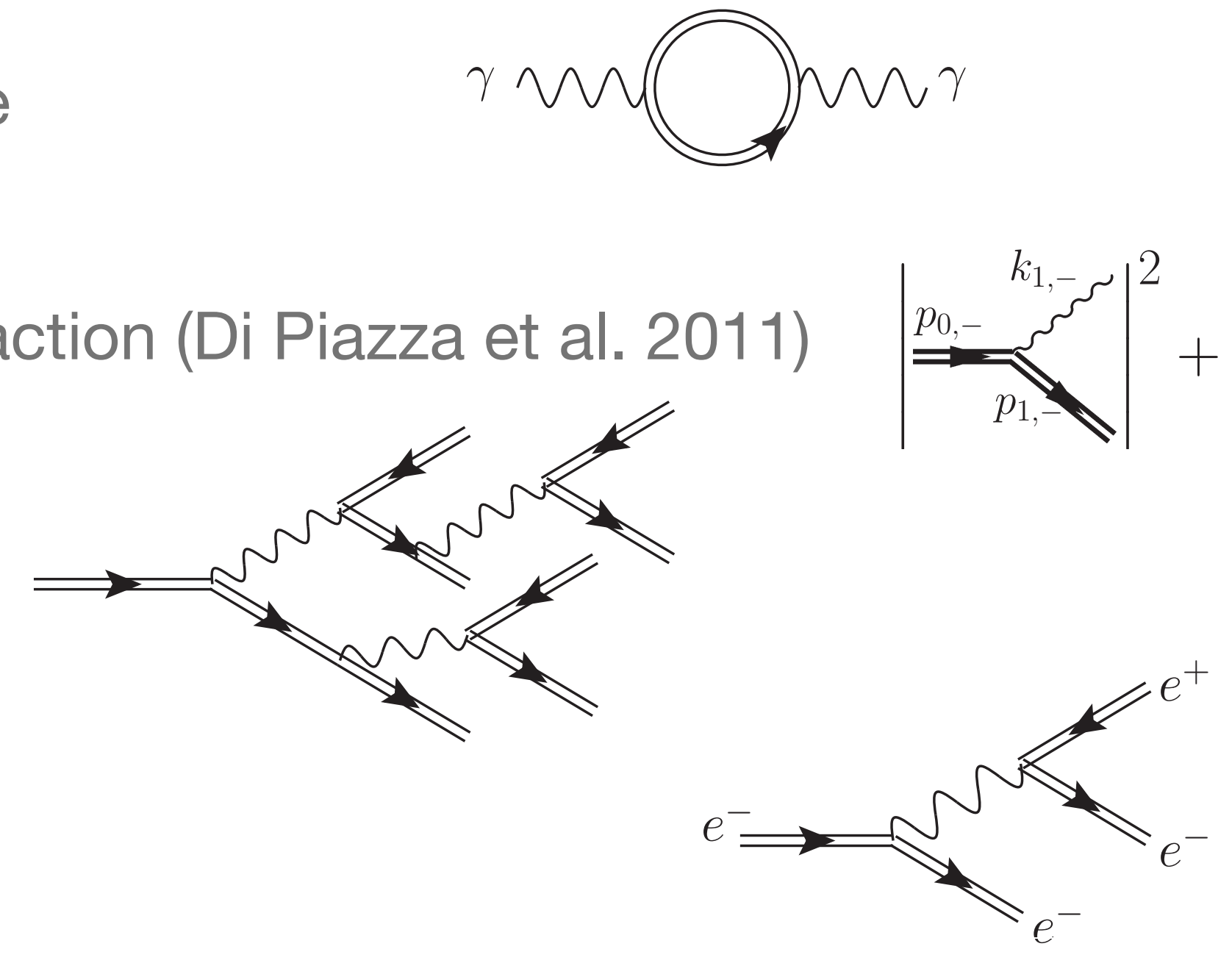


\section{The trident process vs. cascading}

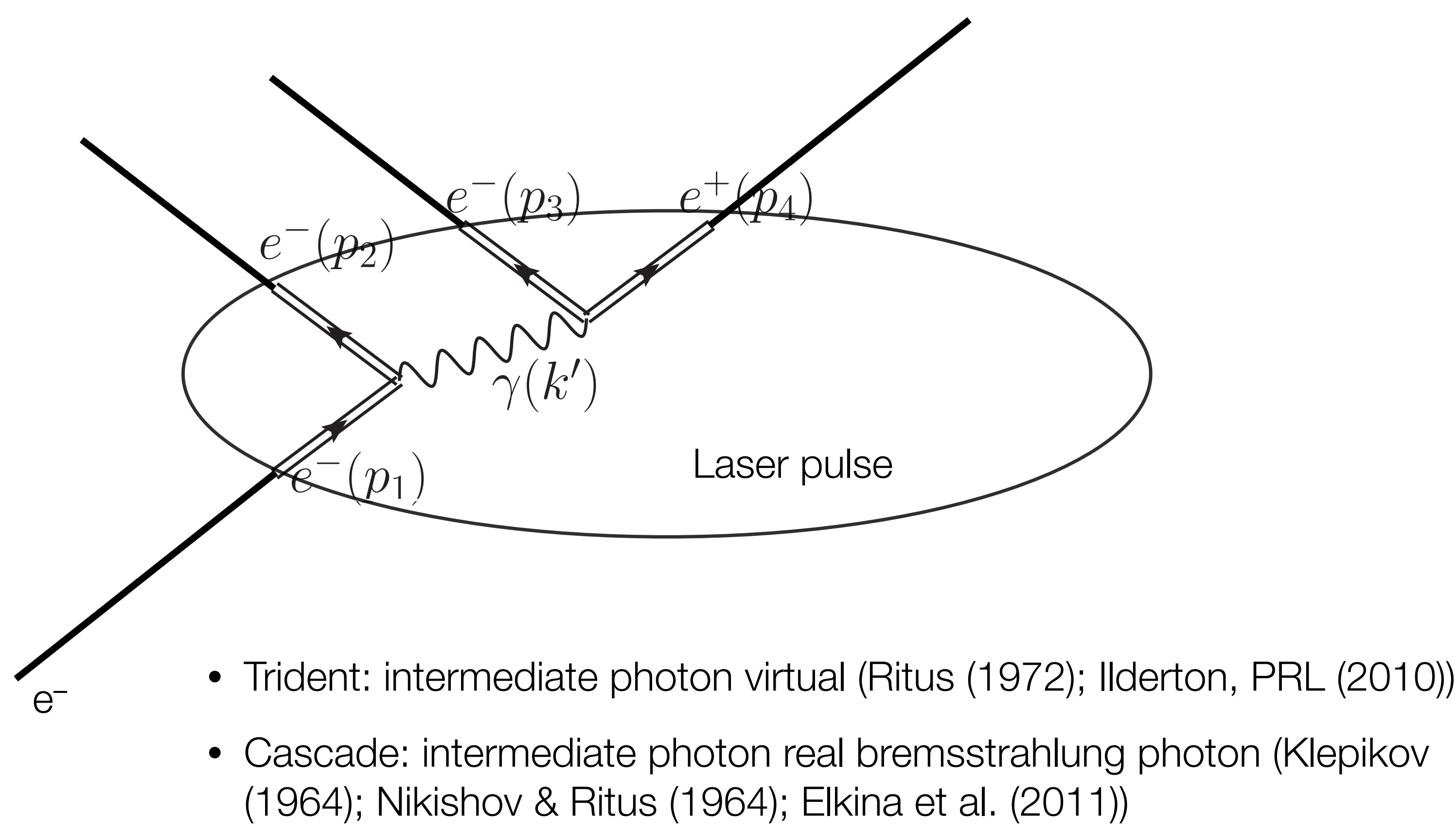




\section{The nonlinear quantum vacuum}

- Special relativity + Heisenberg's uncertainty relation = virtual pair fluctuations.

- Antimatter from Dirac's relativistics quantum mechanics.

- Properly described by QED.

- Photons can effectively interact via fluctuating electron-positron pairs.
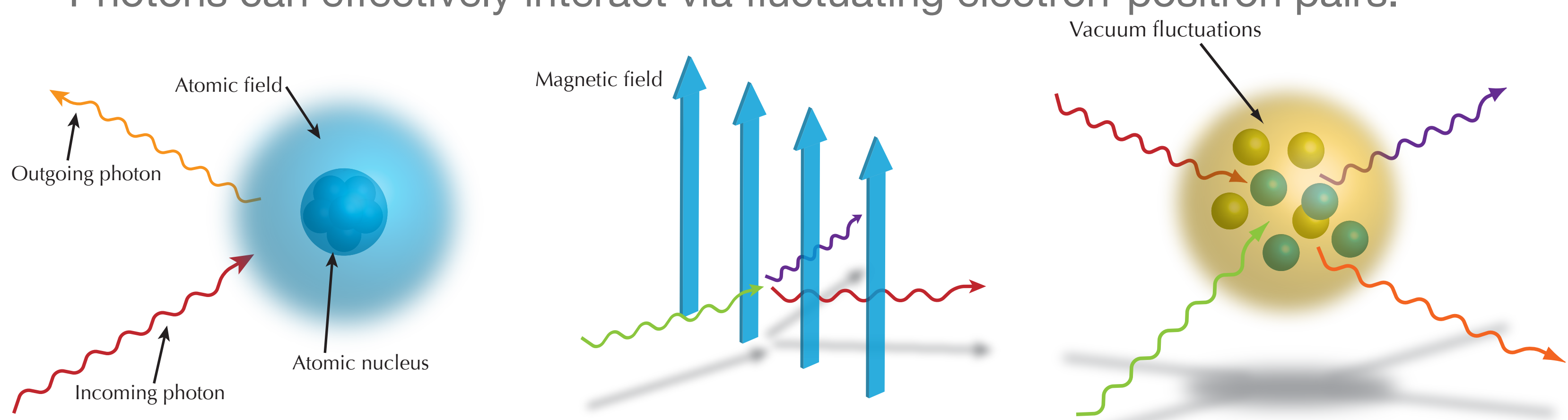

Marklund \& Shukla, Rev. Mod. Phys. 78 (2006); Marklund, Nature Phot. 4 (2010) 


\section{The Heisenberg-Euler Lagrangian}

- Describes the vacuum fluctuations as an effective field theory, fermionic degrees of freedom integrated out.

$$
\begin{aligned}
L & =-\frac{\alpha}{2 \pi} \epsilon_{0} E_{\text {crit }}^{2} \int_{0}^{\mathrm{i} \infty} \frac{d z}{z^{3}} \mathrm{e}^{-z} \times\left[z^{2} \frac{a b}{E_{\text {crit }}^{2}} \operatorname{coth}\left(\frac{a}{E_{\text {crit }}} z\right) \cot \left(\frac{b}{E_{\text {crit }}} z\right)-\frac{z^{2}}{3} \frac{\left(a^{2}-b^{2}\right)}{E_{\text {crit }}^{2}}-1\right] \\
a & =\left[\left(F^{2}+G^{2}\right)^{1 / 2}+F\right]^{1 / 2}, b=\left[\left(F^{2}+G^{2}\right)^{1 / 2}-F\right]^{1 / 2} \\
F & \equiv \frac{1}{2}\left(c^{2} \mathbf{B}^{2}-\mathbf{E}^{2}\right), G \equiv-c \mathbf{E} \cdot \mathbf{B}
\end{aligned}
$$

- Has real and imaginary part. The imaginary part signals depletion, i.e. pair production, the real part defines elastic photon scattering events.

- Can compute equations of motion for test photons in both sub- and supercritical fields. 


\section{Effective Maxwell's equations}

$$
\begin{aligned}
\partial_{a} F^{a b} & =2 \epsilon_{0} \kappa \partial_{a}\left[\left(F_{c d} F^{c d}\right) F^{a b}+\frac{7}{4}\left(F_{c d} \widehat{F}^{c d}\right) \widehat{F}^{a b}\right] \\
\frac{1}{c^{2}} \frac{\partial^{2} \mathbf{E}}{\partial t^{2}}-\nabla^{2} \mathbf{E} & =-\mu_{0}\left[\frac{\partial^{2} \mathbf{P}}{\partial t^{2}}+c^{2} \nabla(\nabla \cdot \mathbf{P})+\frac{\partial}{\partial t}(\nabla \times \mathbf{M})\right] \\
\frac{1}{c^{2}} \frac{\partial^{2} \mathbf{B}}{\partial t^{2}}-\nabla^{2} \mathbf{B} & =\mu_{0}\left[\nabla \times(\nabla \times \mathbf{M})+\frac{\partial}{\partial t}(\nabla \times \mathbf{P})\right] \\
\mathbf{P} & =2 \kappa \epsilon_{0}^{2}\left[2\left(E^{2}-c^{2} B^{2}\right) \mathbf{E}+7 c^{2}(\mathbf{E} \cdot \mathbf{B}) \mathbf{B}\right] \\
\mathbf{M} & =2 \kappa \epsilon_{0}^{2} c^{2}\left[-2\left(E^{2}-c^{2} B^{2}\right) \mathbf{B}+7(\mathbf{E} \cdot \mathbf{B}) \mathbf{E}\right]
\end{aligned}
$$

Bialynicka-Birula \& Bialynicki-Birula (1970) 


\section{Dispersive corrections to HE-lagrangian}

$$
\mathcal{L}_{D}=\sigma \epsilon_{0}\left[\left(\partial_{a} F^{a b}\right)\left(\partial_{c} F_{b}^{c}\right)-F_{a b} \square F^{a b}\right]
$$

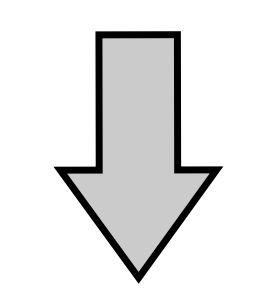

$\frac{1}{2}(1+2 \sigma \square) \partial_{a} F^{a b}=\epsilon_{0} \kappa \partial_{a}\left[\left(F_{c d} F^{c d}\right) F^{a b}+\frac{7}{4}\left(F_{c d} \widehat{F}^{c d}\right) \widehat{F}^{a b}\right]$

Here $\sigma=(2 / 15) \alpha c^{2} / \omega_{e}^{2} \approx 1.4 \times 10^{-28} \mathrm{~m}^{2}$ 


\section{Dispersion relations in external fields}

- Result due to HE interactions; nonlinear function of field strengths

$$
\omega \approx c|\mathbf{k}|\left(1-\frac{1}{2} \lambda|\mathbf{Q}|^{2}\right) \quad|\mathbf{Q}|^{2} \equiv \epsilon_{0}|\hat{\mathbf{k}} \times \mathbf{E}+c \hat{\mathbf{k}} \times(\hat{\mathbf{k}} \times \mathbf{B})|^{2}
$$

$$
\lambda \sim \frac{\alpha}{90 \pi} \frac{1}{E_{\text {crit }}}
$$

- Result due to HE interactions + lowest order corrections due to finite frequency (still perturbative); nonlinear function of wavenumber

$$
\omega \approx c|\mathbf{k}|\left[1-\frac{1}{2} \lambda|\mathbf{Q}|^{2}\left(1+2 \sigma \lambda|\mathbf{Q}|^{2}|\mathbf{k}|^{2}\right)\right]
$$

- Dispersion relation: Hamiltonian for photons. $\quad \dot{x}=\frac{\partial \omega}{\partial k} \quad \dot{k}=-\frac{\partial \omega}{\partial x}$

- Side note: phase and group velocites may change in external fields, Cherenkov radiation due to the quantum vacuum (MM et al. 2005). 
The nonlinear quantum vacuum: photon-photon scattering

$$
\begin{aligned}
& \sigma_{\gamma \gamma} \approx 0.7 \times 10^{-29}\left(\frac{\hbar \omega}{1 \mathrm{MeV}}\right)^{6} \mathrm{~cm}^{2} \\
& \mathcal{L}=\mathcal{L}_{0}+\frac{\epsilon_{0} \alpha}{90 \pi E_{\mathrm{crit}}^{2}}\left[\left(E^{2}-c^{2} B^{2}\right)^{2}+7 c^{2}(\boldsymbol{E} \cdot \boldsymbol{B})^{2}\right]
\end{aligned}
$$

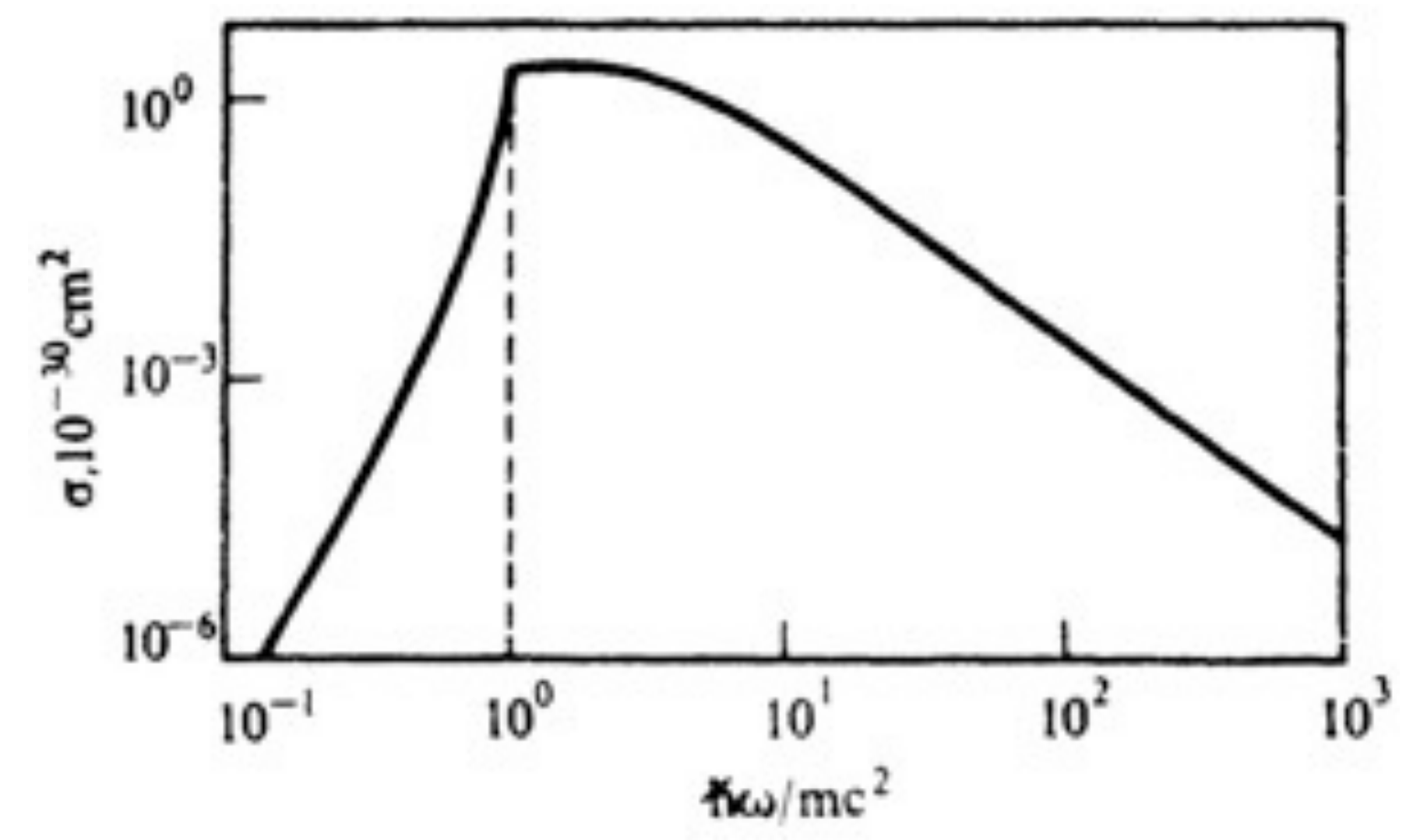




\section{The nonlinear quantum vacuum: photon-photon scattering}

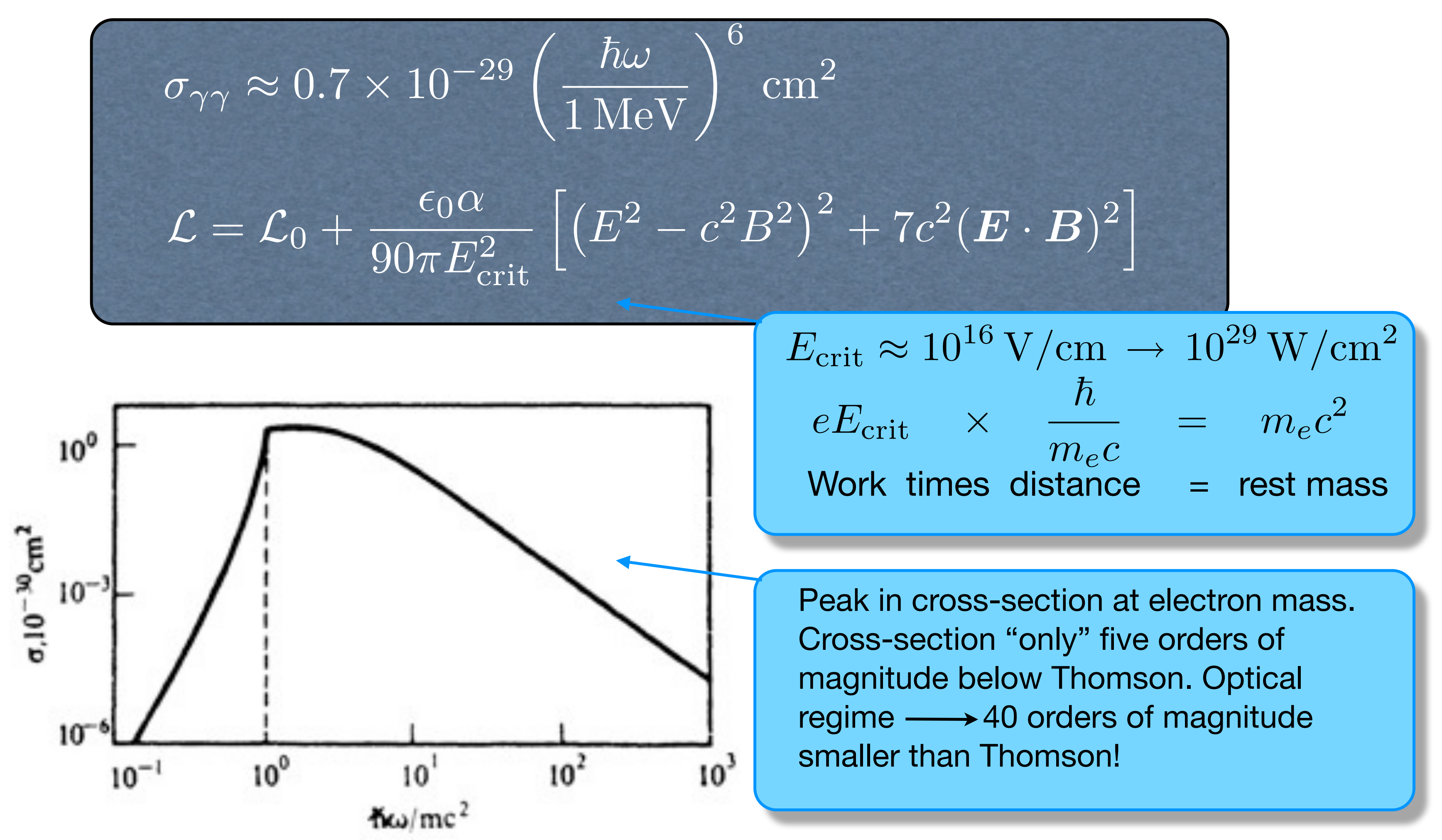




\section{Vacuum birefringence}

- Anisotropic vacuum, due to e.g. magnetic field (Adler 1970, 1971, Heyl \& Hernquist 1997, Dittrich \& Gies 1998, Rikken \& Rizzo 2000, 2003); permittivity and permeability

$$
\begin{aligned}
\epsilon_{i j} & =\delta_{i j}+\frac{4 \alpha}{90 \pi} \frac{B^{2}}{E_{\mathrm{crit}}^{2}}\left(-\delta_{i j}+\frac{7}{2} b_{i} b_{j}\right) \\
\mu_{i j} & =\delta_{i j}+\frac{4 \alpha}{90 \pi} \frac{B^{2}}{E_{\mathrm{crit}}^{2}}\left(\delta_{i j}+2 b_{i} b_{j}\right)
\end{aligned}
$$

- Refractive index different for different propagation angles, relative external field

$$
n-1 \sim \frac{\alpha}{90 \pi} \frac{B^{2}}{E_{\text {crit }}^{2}} \sin ^{2} \theta
$$




\section{Vacuum birefringence}

- Difference $\Delta n$ in parallel and perpendicular refractive index will yield cummulative ellipticity over propagation distance $L$ (Bakalov et al. 1998)

$$
\Psi=\frac{\pi L}{\lambda} \Delta n \sin (2 \theta)
$$

- Quite difficult to measure; strong magnetic fields may interfer with measurements, shielding difficult.

- Can also consider using coupled Fabry-Perot cavities, where one provides the external field, while the other supplies the probe photon (Luiten \& Petersen 2004)

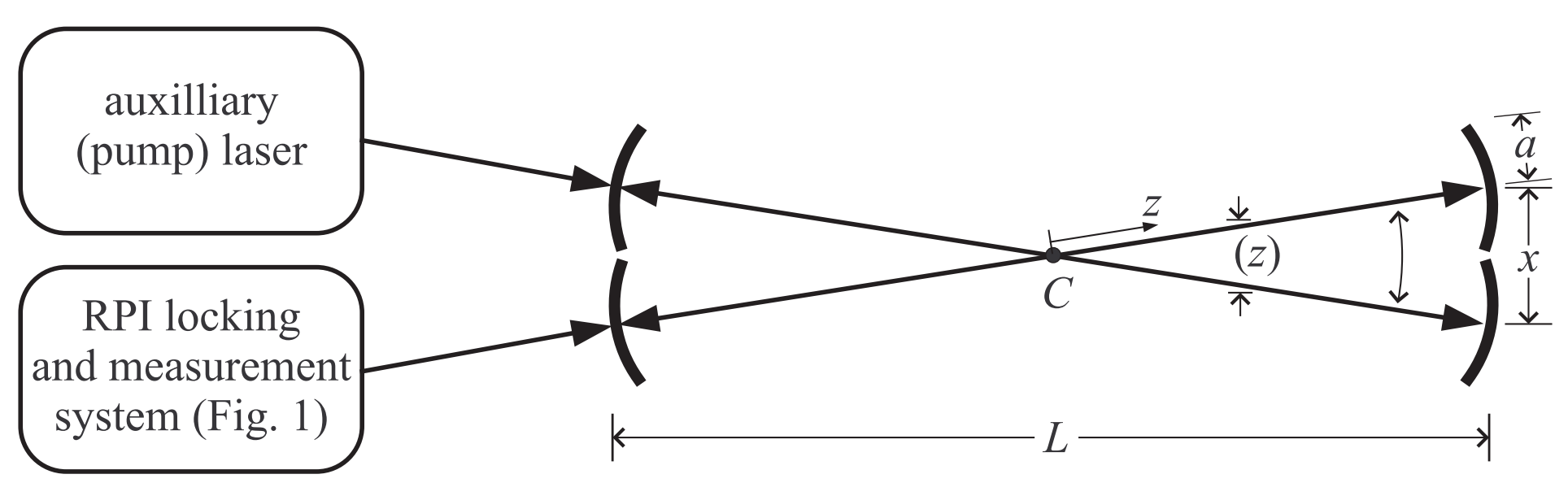




\section{Cavity experiments}

- High-field cavities (around 50MV), far from Schwinger limit.

- Can support only certain modes, filtering. Need super cooling (1 K).

- Generation of harmonics through vacuum fluctuations. beats thermal effects.

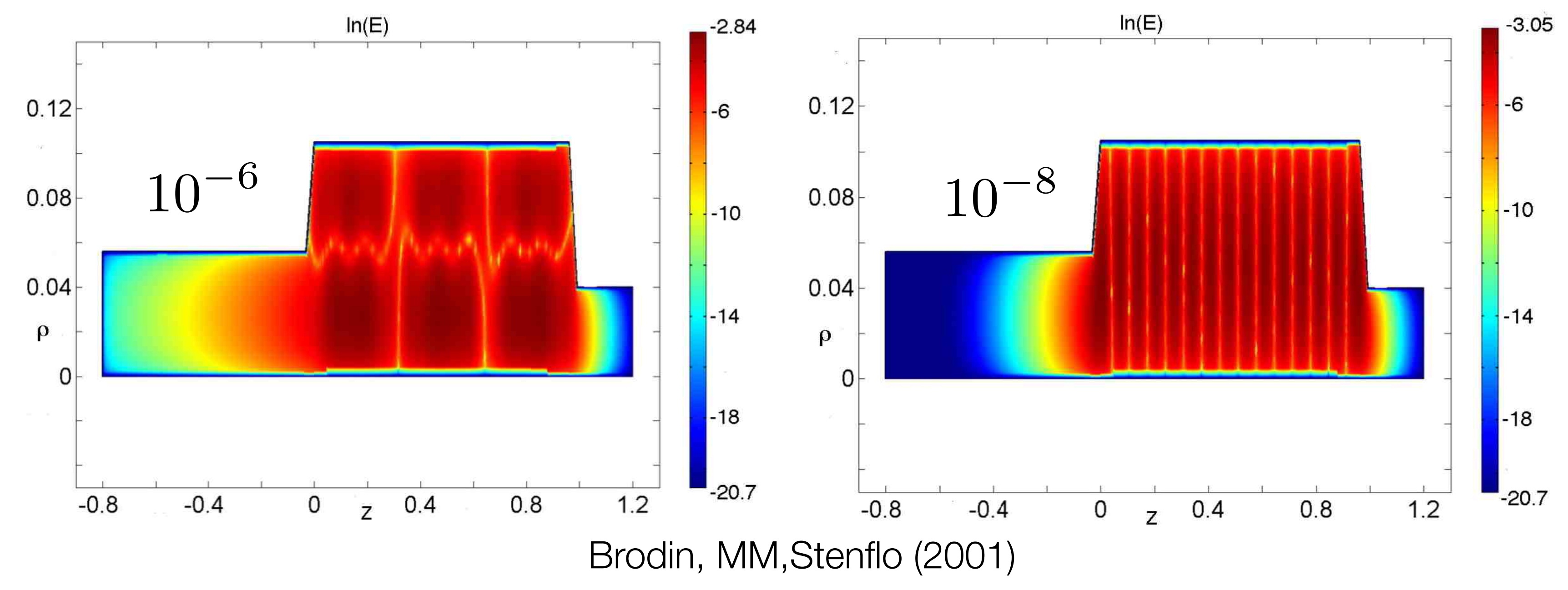




\section{Cavity experiments}

- High-field cavities (around 50MV), far from Schwinger limit.

- Can support only certain modes, filtering. Need super cooling (1 K).

- Generation of harmonics through vacuum fluctuations, beats thermal effects.

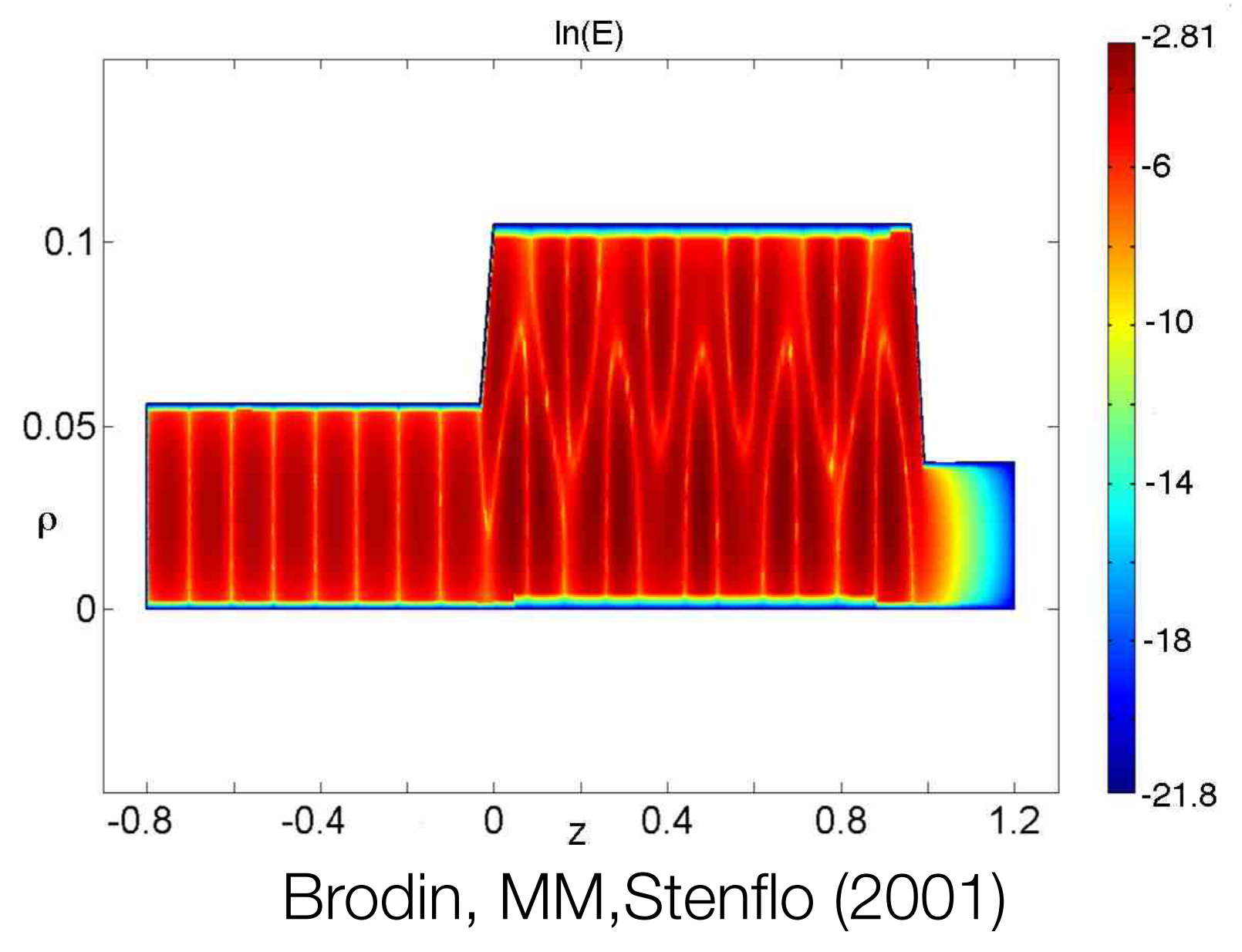




\section{The nonlinear quantum vacuum: photon-photon scattering}

- Photon-photon scattering for low-energy photons $\hbar \omega \ll 2 m_{e} c^{2}$

- Why? Just to confirm QED?

- Testing the nonlinear coefficients in the Heisenberg-Euler lagrangian.

- Compare, e.g., Born-Infeld type lagrangian (Lorentz invariant, Born \& Infeld 1934; no birefringence in static fields (unique property))

$$
\mathcal{L}_{\mathrm{BI}}=\kappa^{2}\left[1-\sqrt{1+\frac{1}{2 \kappa^{2}}\left(E^{2}-B^{2}\right)-\frac{1}{16 \kappa^{2}}(\mathbf{E} \cdot \mathbf{B})^{2}}\right]
$$

- (Finite field energies around point charges) 


\section{The nonlinear quantum vacuum: photon-photon scattering}

- Use high-power laser system (1019-1020 photons) to overcome miniscule scattering cross section.

- Could be detectable (Bernard et al. (1998); Lundström et al., PRL 96 (2006)).

$$
\begin{aligned}
& \mathbf{k}_{1}+\mathbf{k}_{2}=\mathbf{k}_{3}+\mathbf{k}_{4} \\
& \omega_{1}+\omega_{2}=\omega_{3}+\omega_{4}
\end{aligned}
$$

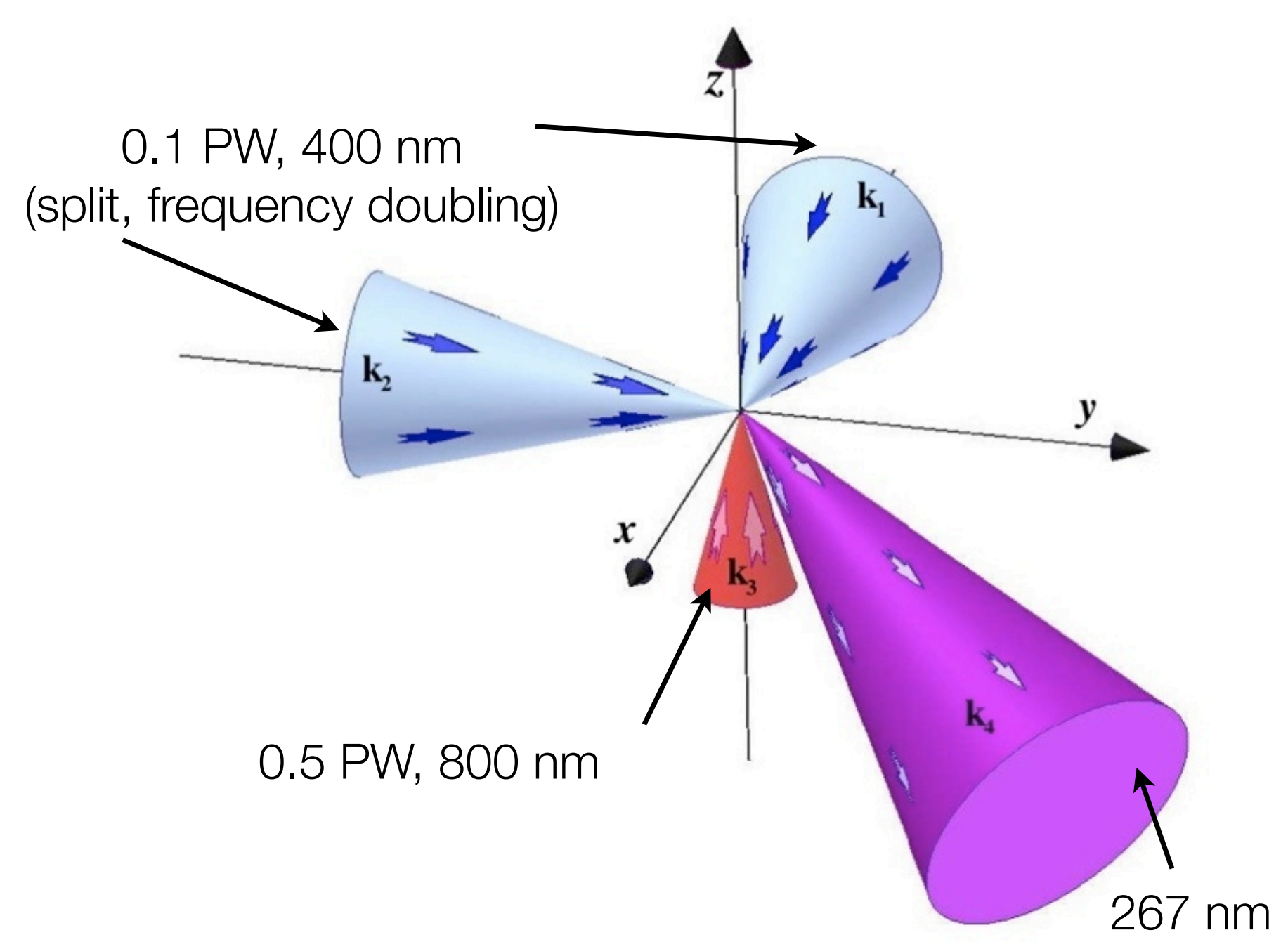




\section{The nonlinear quantum vacuum: photon-photon scattering}

- Use high-power laser system (1019-1020 photons) to overcome miniscule scattering cross section.

- Could be detectable (Bernard et al. (1998); Lundström et al., PRL 96 (2006)).

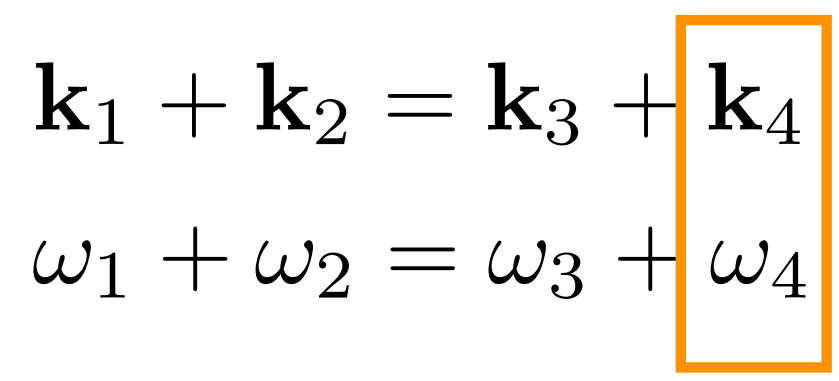

Generated photons
$0.1 \mathrm{PW}, 400 \mathrm{~nm}$

(split, frequency doubling)

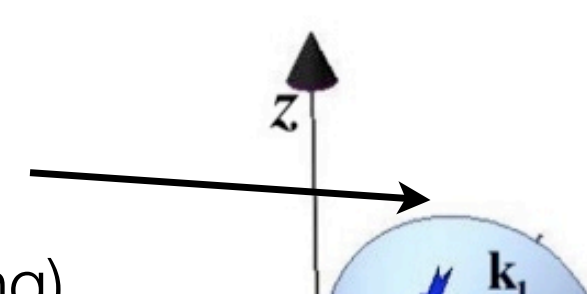

$0.5 \mathrm{PW}, 800 \mathrm{~nm}$

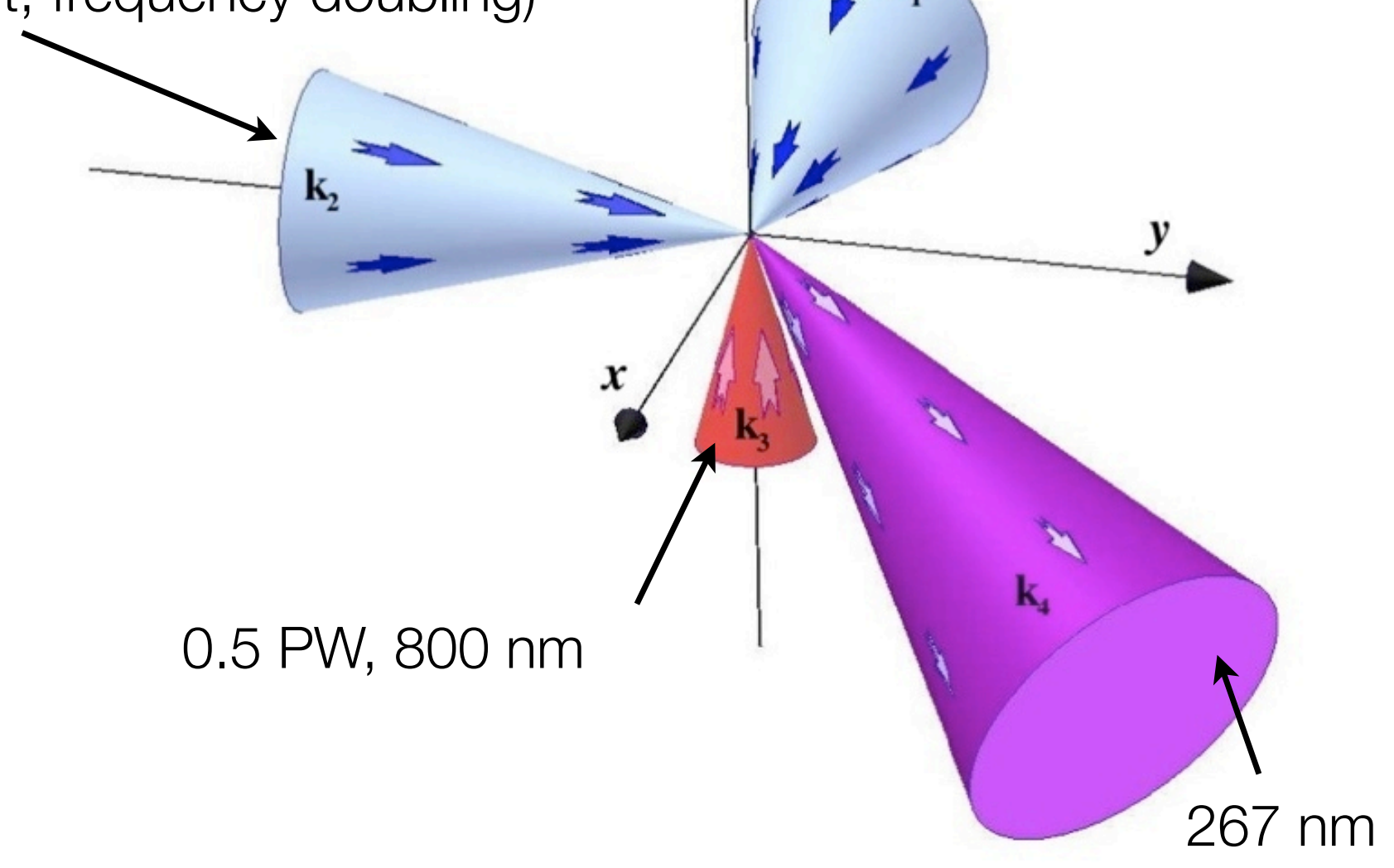




\section{The nonlinear quantum vacuum: photon-photon scattering}

- Number of generated photons as a function of beam power.

$$
N \approx G \times 10^{-2}\left(\frac{1 \mu \mathrm{m}}{\lambda_{4}}\right)^{3}\left(\frac{L}{1 \mu \mathrm{m}}\right)\left(\frac{P_{1} P_{2} P_{3}}{1 \mathrm{PW}^{3}}\right)
$$

Form factor depending on pulse shape etc. Of order unity

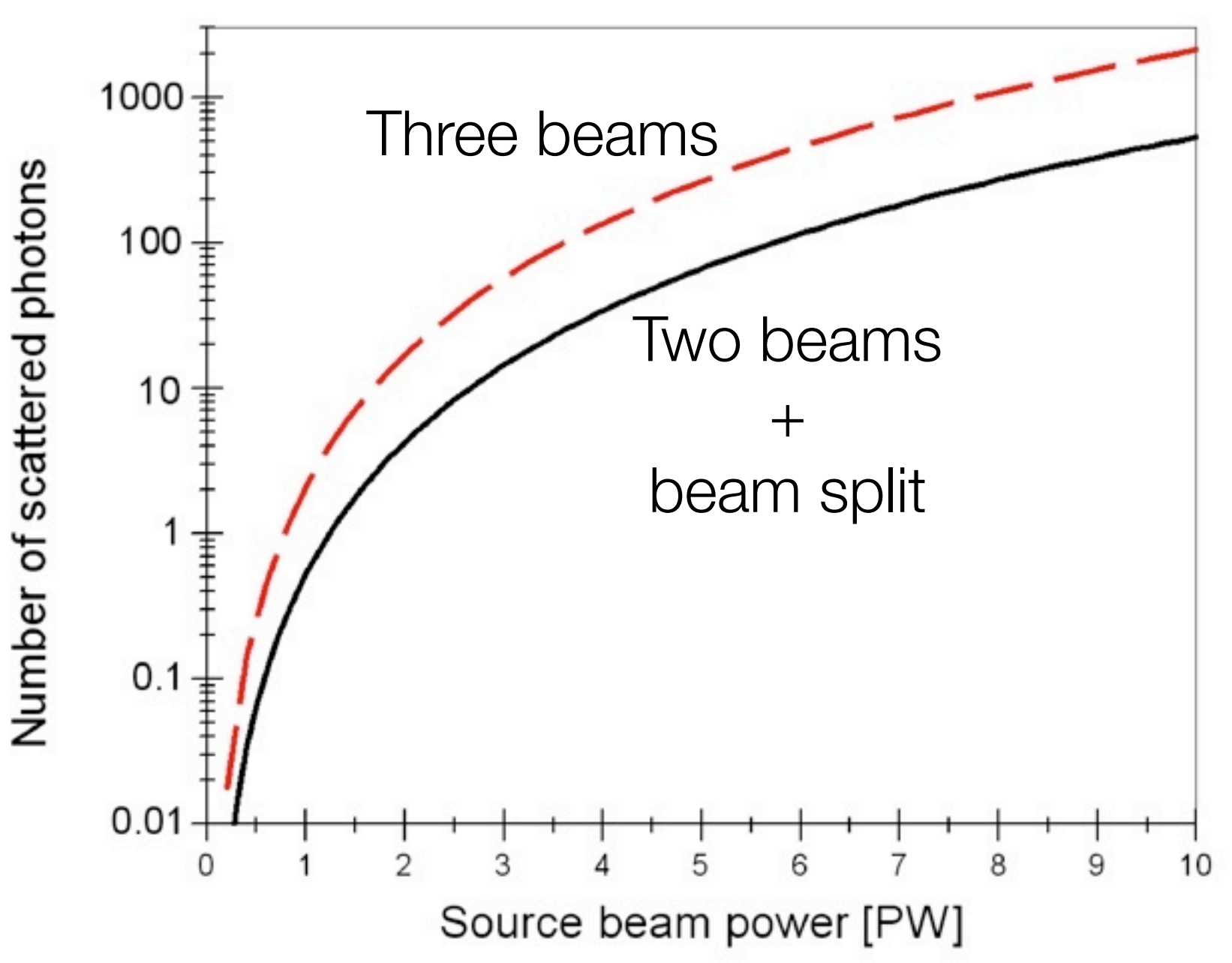




\section{The nonlinear quantum vacuum: photon-photon scattering}

- Number of generated photons as a function of beam power.

$$
N \approx G \times 10^{-2}\left(\frac{1 \mu \mathrm{m}}{\lambda_{4}}\right)^{3}\left(\frac{L}{1 \mu \mathrm{m}}\right)\left(\frac{P_{1} P_{2} P_{3}}{1 \mathrm{PW}^{3}}\right)
$$

Form factor depending on pulse shape etc. Of order unity

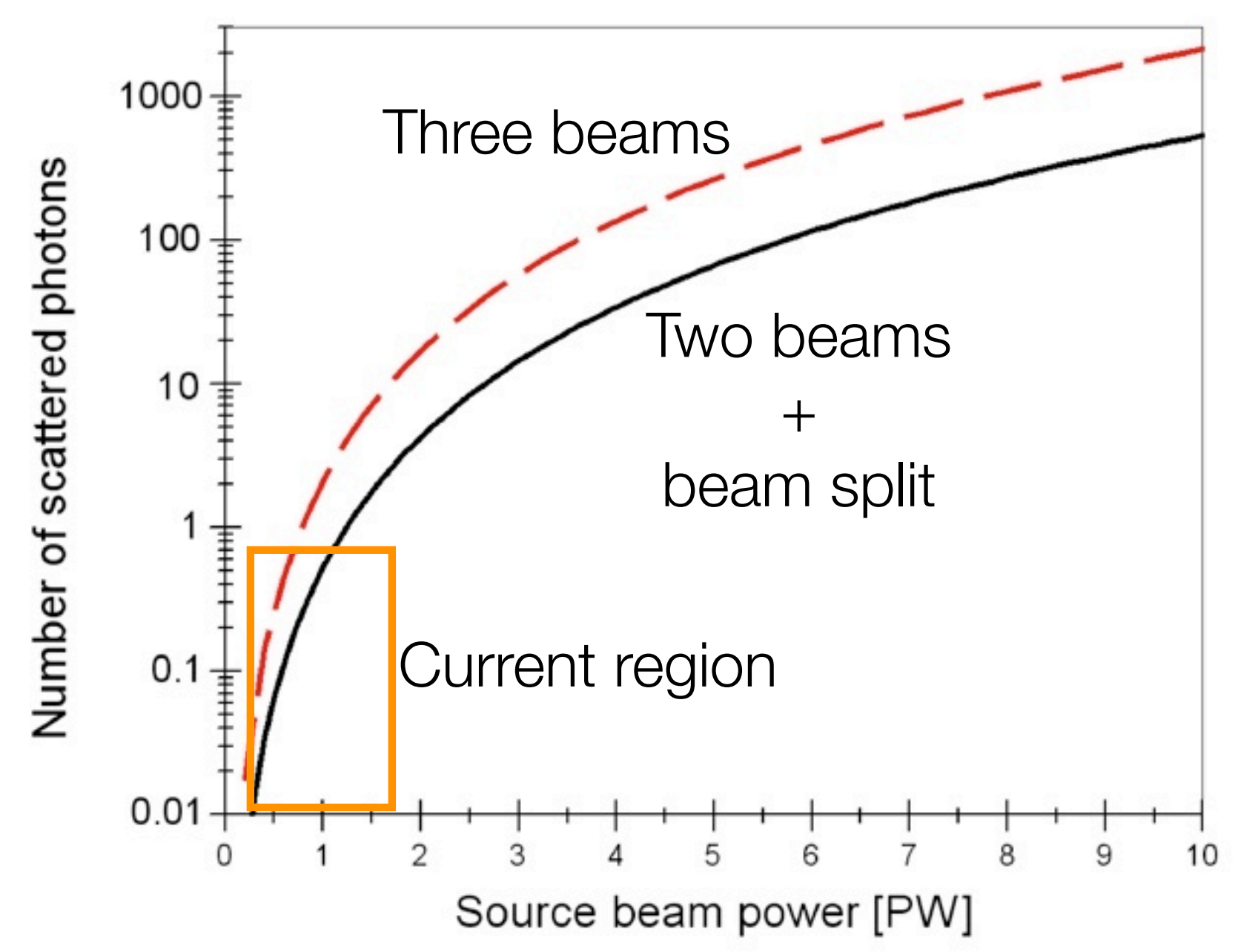




\section{The nonlinear quantum vacuum: photon-photon scattering}

- Number of generated photons as a function of beam power.

$$
N \approx G \times 10^{-2}\left(\frac{1 \mu \mathrm{m}}{\lambda_{4}}\right)^{3}\left(\frac{L}{1 \mu \mathrm{m}}\right)\left(\frac{P_{1} P_{2} P_{3}}{1 \mathrm{PW}^{3}}\right)
$$

Form factor depending on pulse shape etc. Of order unity

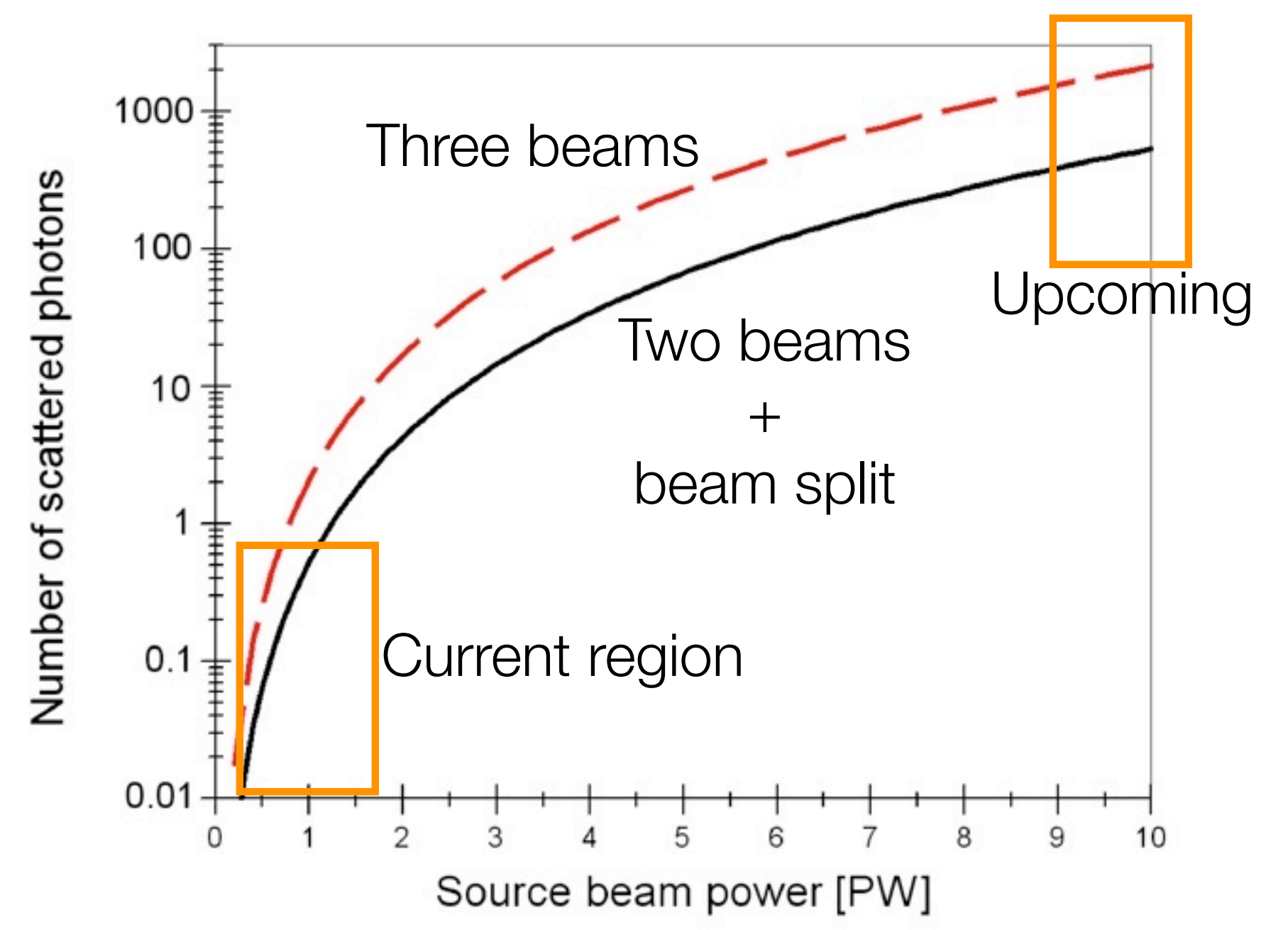




\section{The nonlinear quantum vacuum: photon-photon scattering}

- Alternative setup.

- Larger geometric factor.

- Smaller interaction volume.

- Higher harmonics in directions of generated photons!

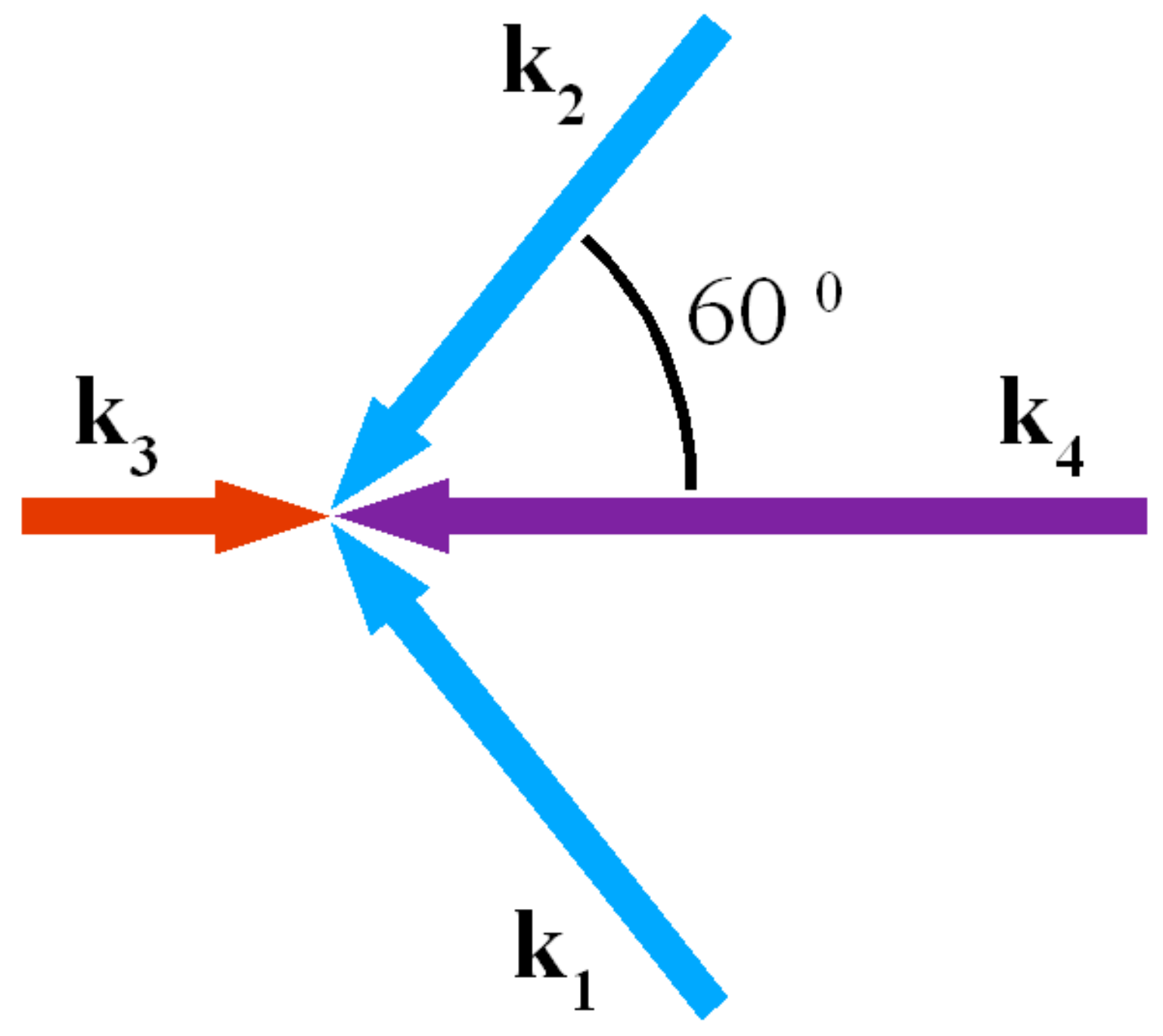




\section{The nonlinear quantum vacuum: photon-photon scattering}

- Noise sources:

- Compton scattering of electrons.

- Collective plasma effects: harmonics (imperfect vacuum).

- Photon scattering in chamber.

- Thermal noise. 


\section{The nonlinear quantum vacuum: photon-photon scattering}

- Virtual slit experiments (King et al., Nature Phot. 4 (2010))

- Interference pattern, like in double slit experiment.

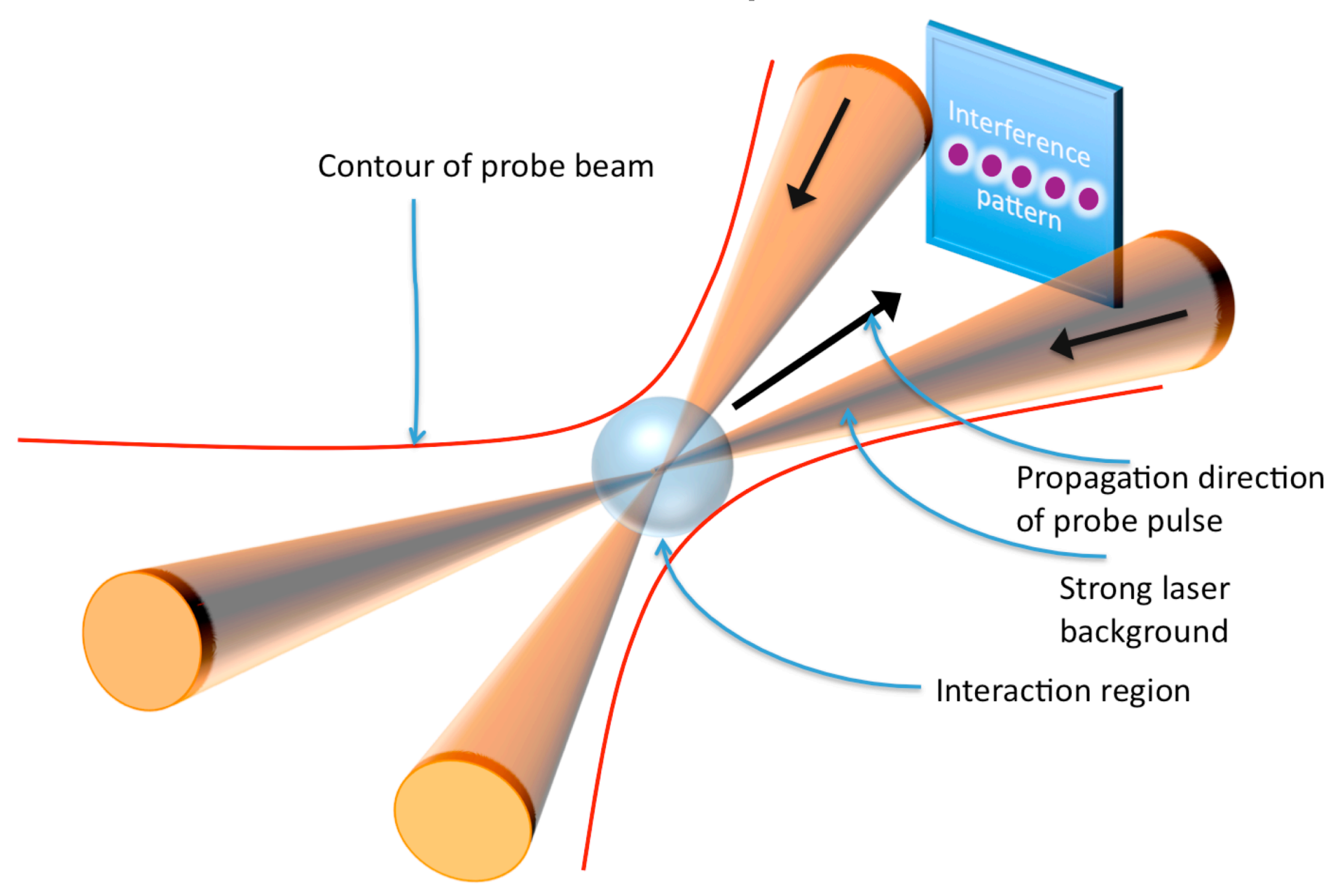




\section{Photon splitting}

Photons down-converted in strong vacuum magnetic field ("photon splitting") (Adler 1971). (See talks by Gies and Heyl)
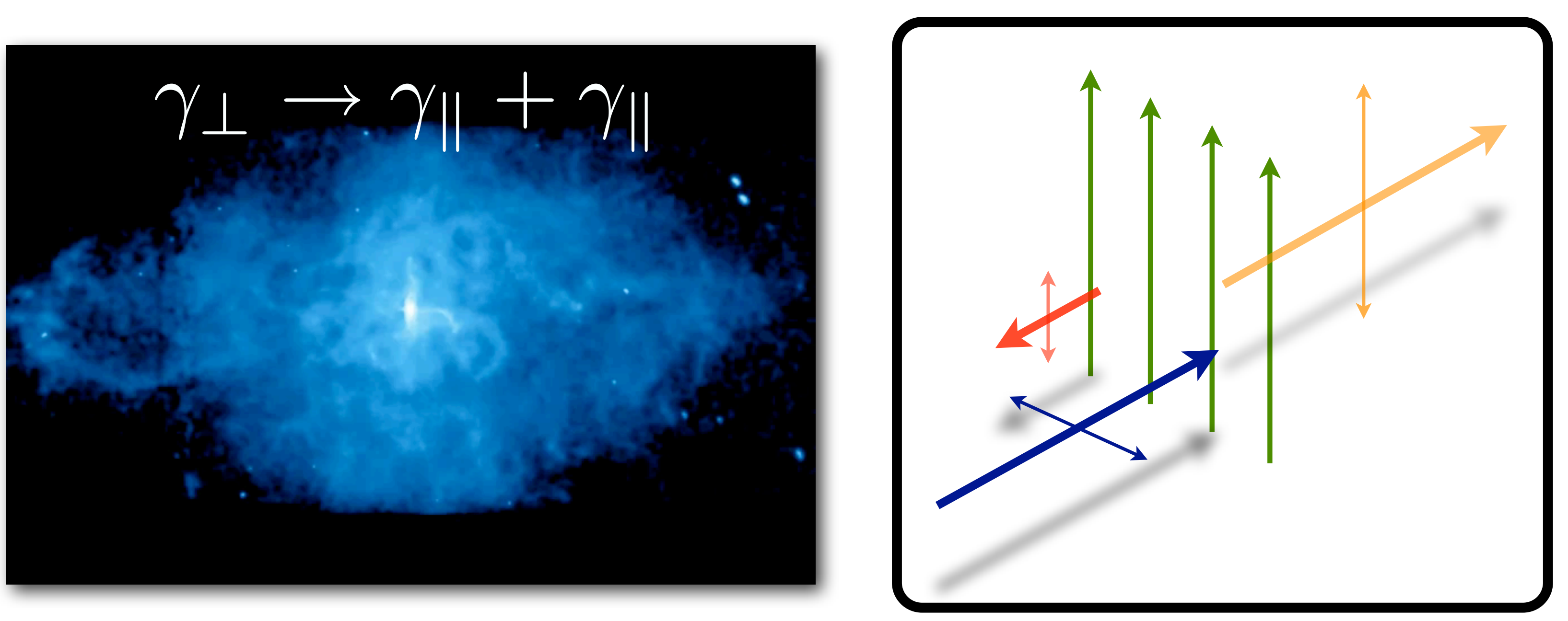
Photon splitting in plasmas

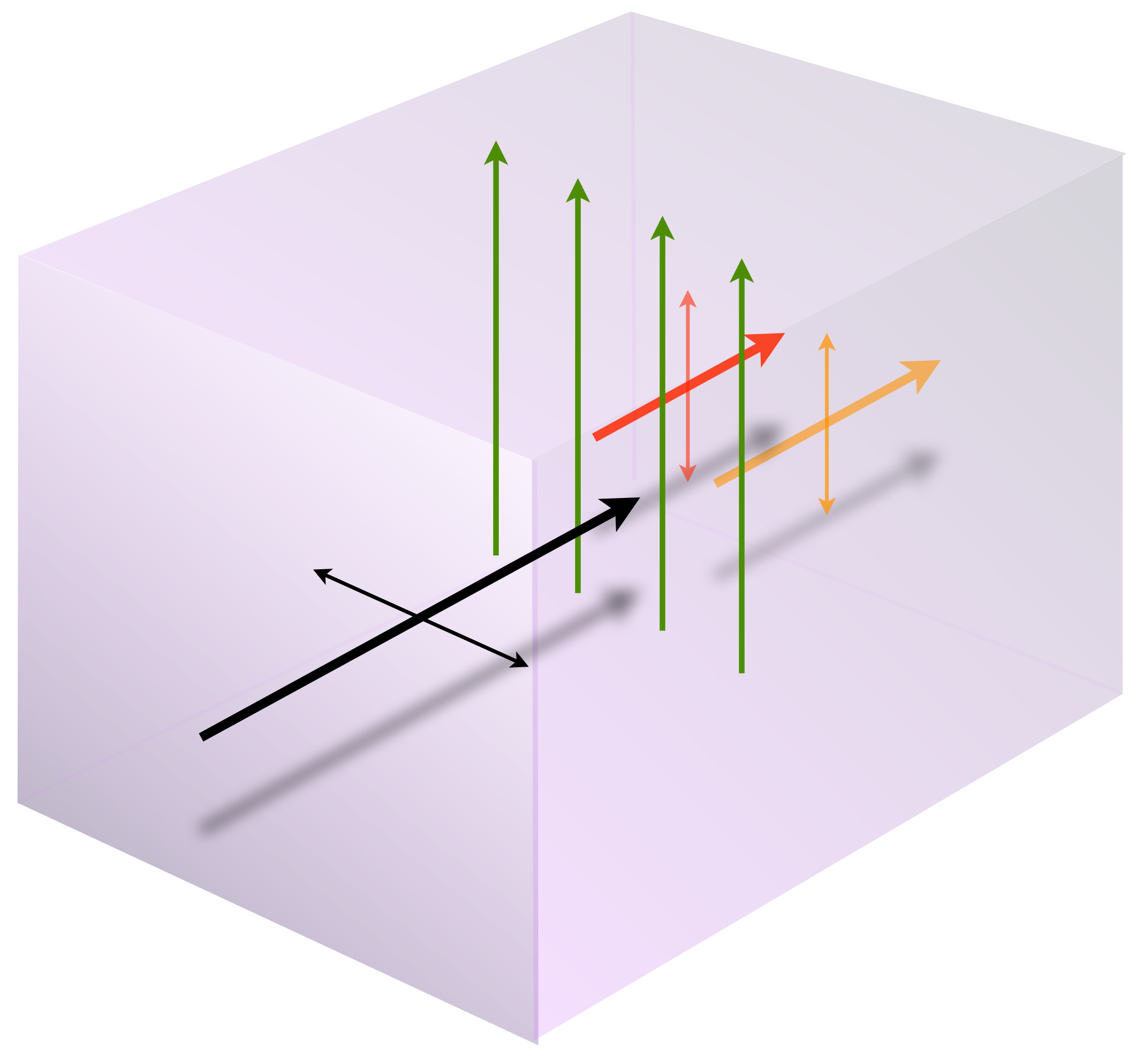




\section{Photon splitting in plasmas}

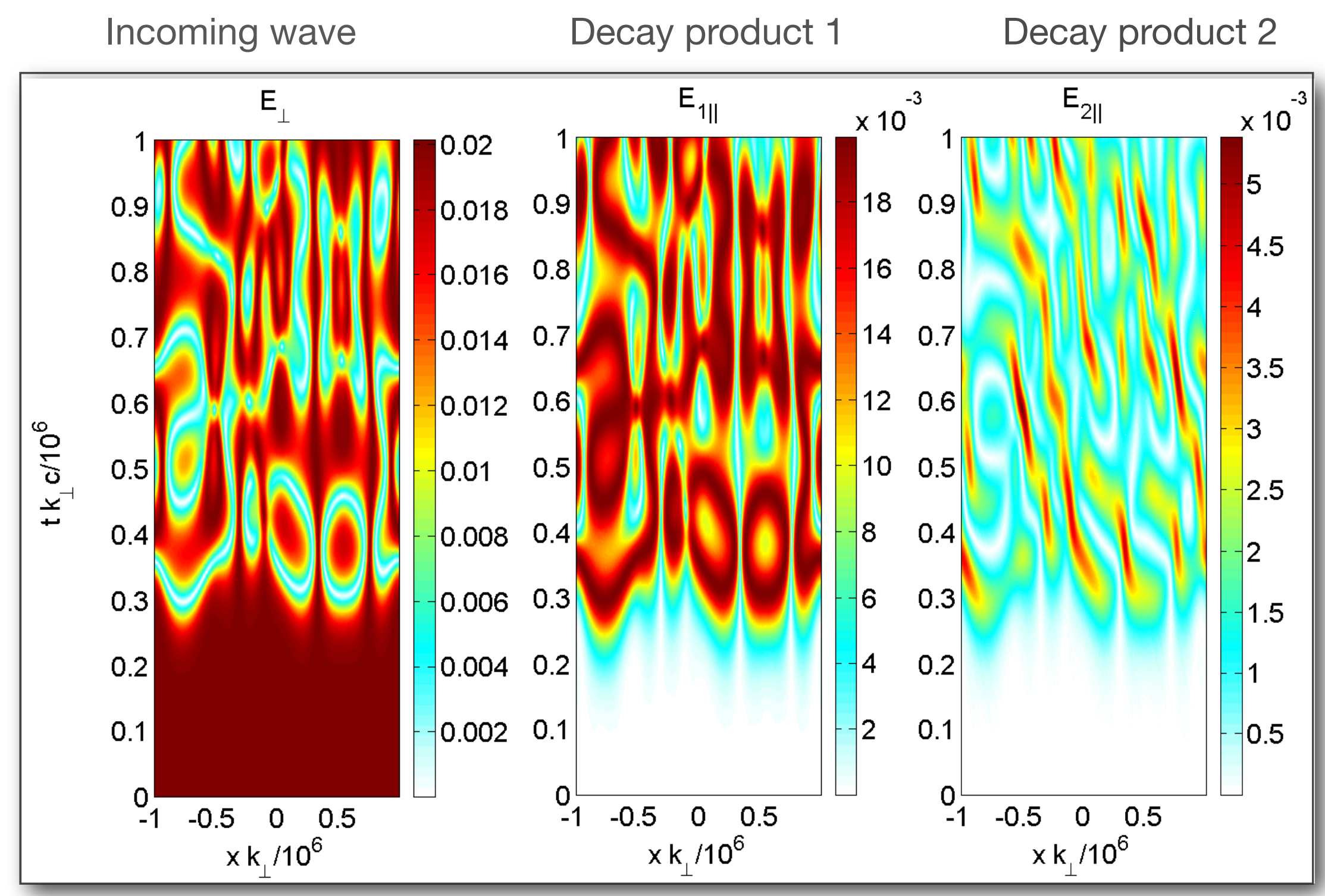

(Brodin, MM et al. 2007) 


\section{Generation of vacuum harmonics}

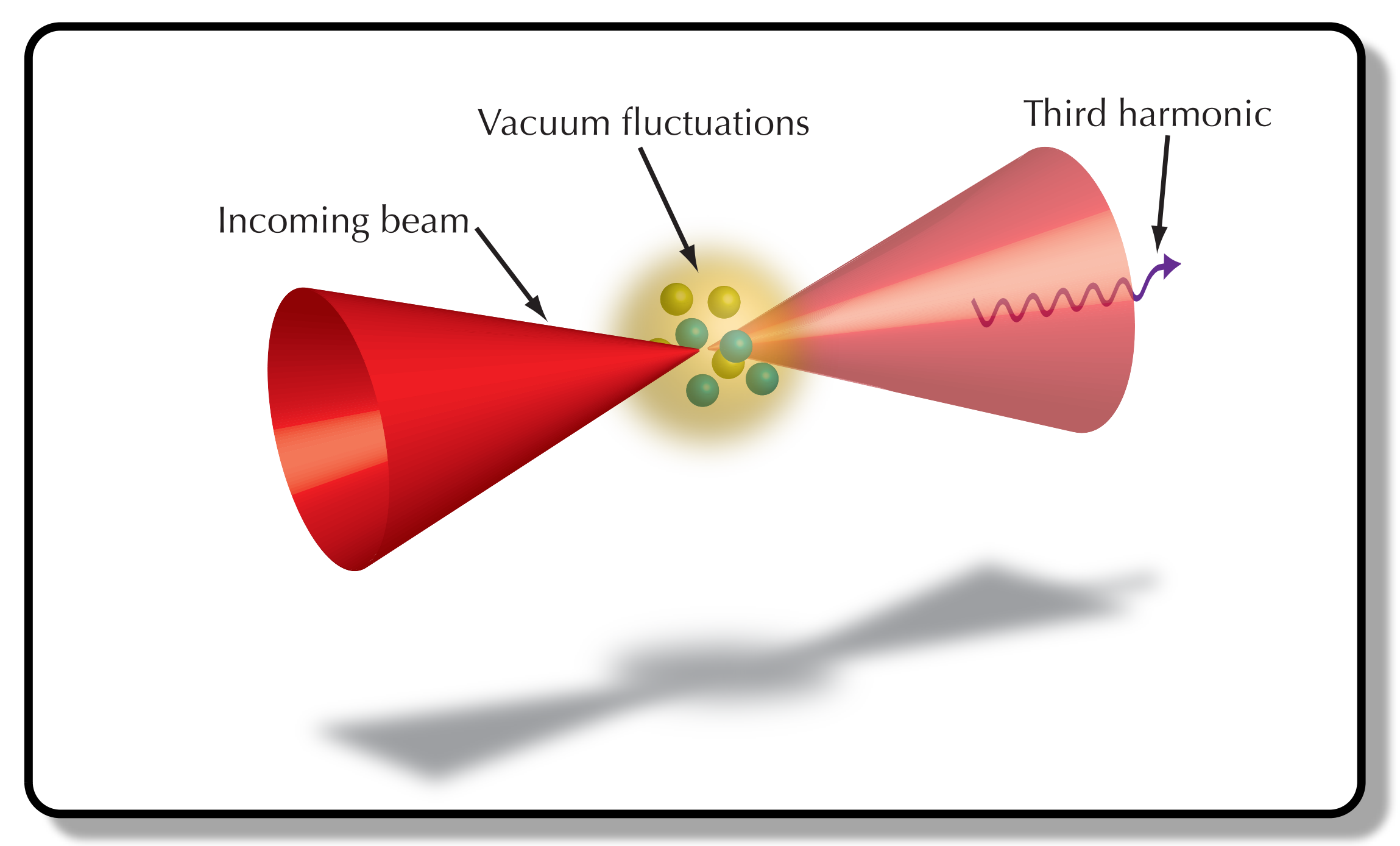

- Harmonics "possible" due to non-planar structure of laser pulses or other external broken symmetries (Ding \& Kaplan 1990, Di Piazza et al. 2005, Fedotov \& Narozhny 2007)

- Nonlinear and nonperturbative surprises (as in laser-plasma case)? 


\section{Colliding pulses}

- Two colliding pulse, criteria for collapse (nonlinear refractive index)

$$
\left(\frac{\alpha}{90 \pi}\right)^{1 / 2} \frac{E}{E_{\text {crit }}}>\frac{r_{p}}{\ell_{p}}
$$

- Long pulse, i.e., large energy.

- Diffraction limit: $\quad r_{p} \sim \lambda_{p}$

- For intensities two orders of magnitude

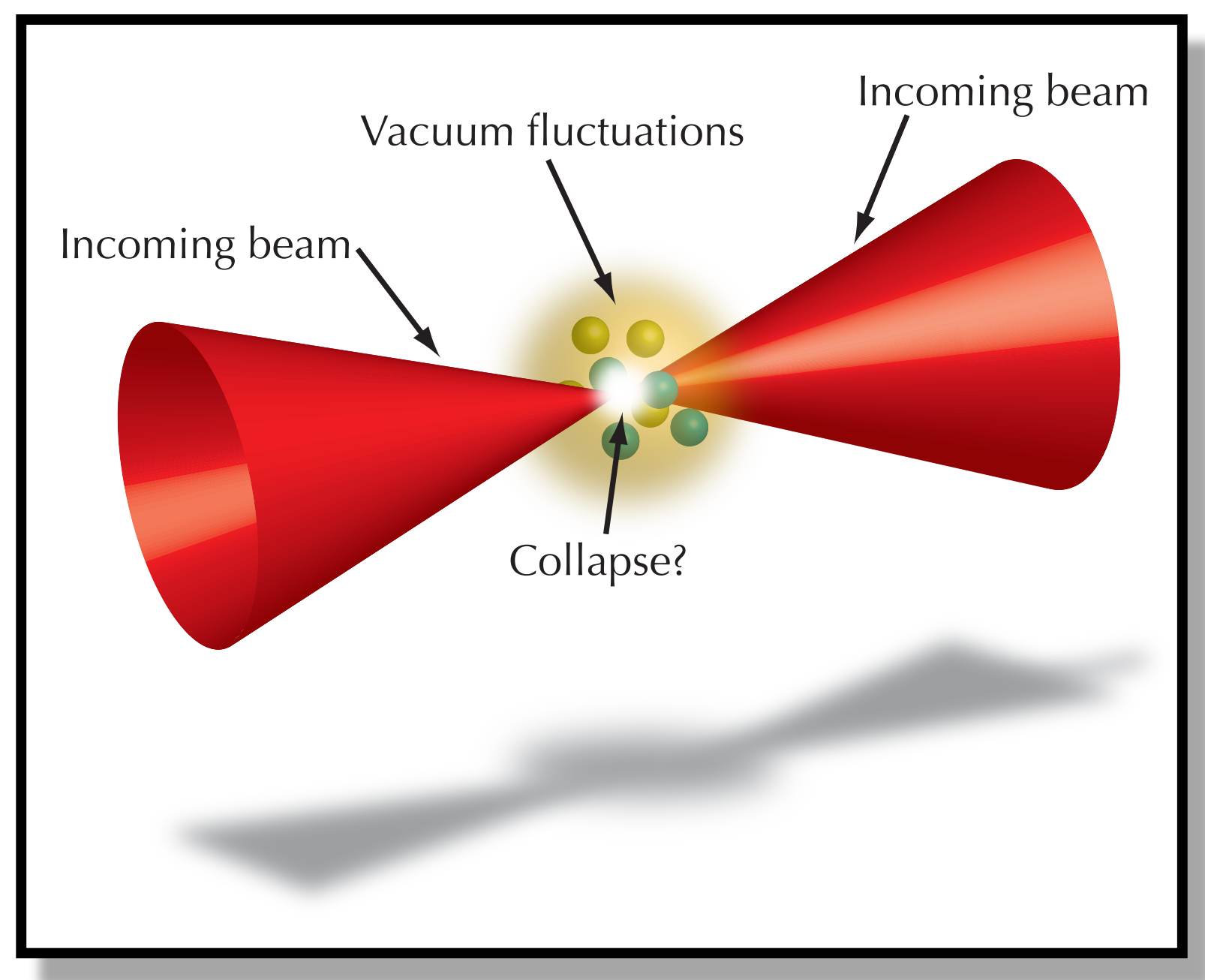
below Schwinger intensity:

$$
\ell_{p} \sim 10^{4} \lambda_{p}
$$




\section{Interaction between coherent and incoherent photons: radiation gas}

- Evolution determined by nonlinearly coupled system, much like a laserplasma (MM 2003)

$$
\begin{aligned}
& i \partial_{t} E+\beta \nabla^{2} E+\kappa \varepsilon E=0 \\
& \left(\partial_{t}^{2}-\frac{1}{3} \nabla^{2}\right) \varepsilon=\mu \nabla^{2}|E|^{2}
\end{aligned}
$$

- Nonlinear Schrödinger equation: know that such systems yields solitons, pulse collapse and filamentations. 


\section{Interaction between coherent and incoherent photons: radiation gas}

Far away from being detectable! 


\section{Interaction between coherent and incoherent photons: radiation gas}
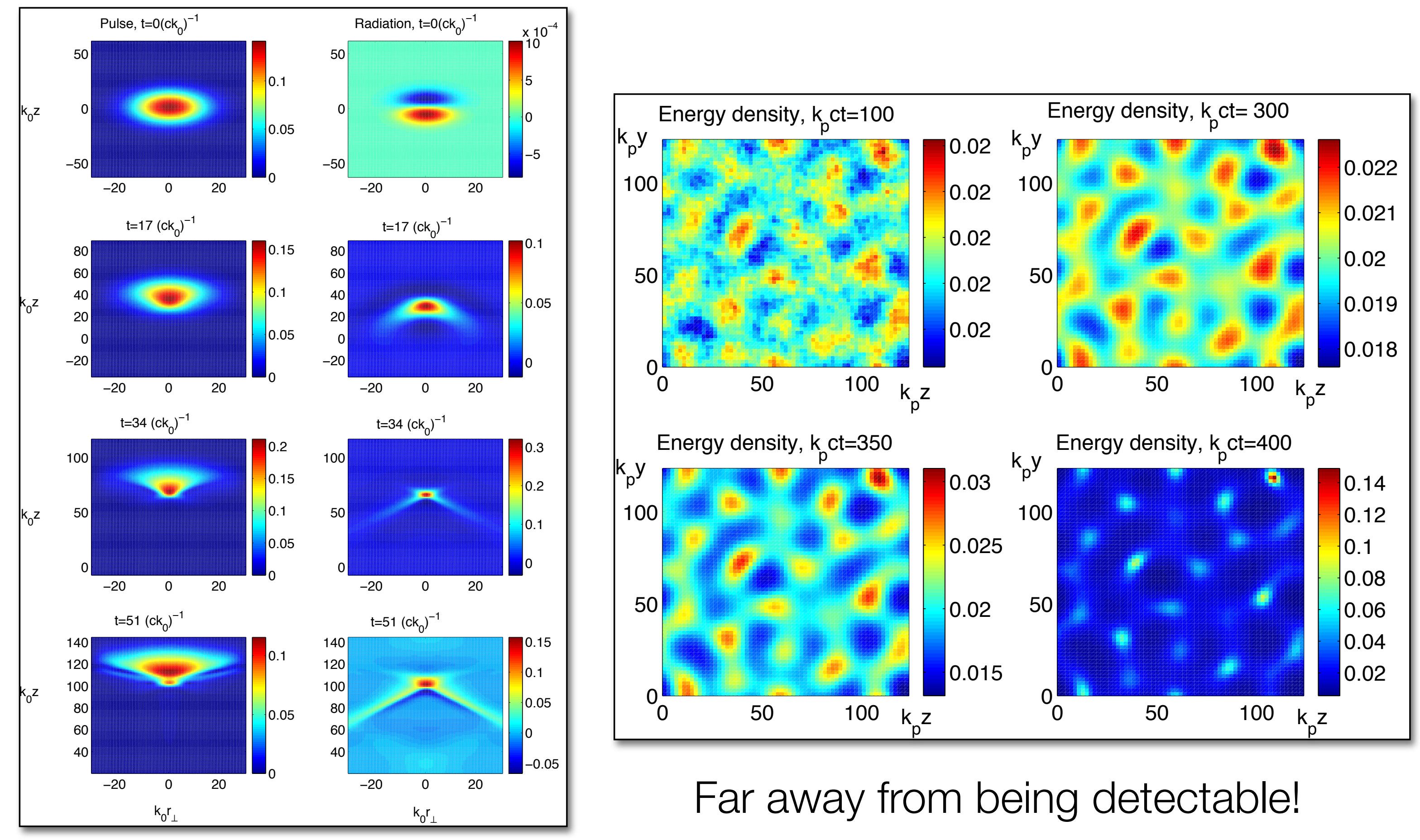

Far away from being detectable! 


\section{The Scharnhorst effect}

- The results of Casimir.

- Fewer vacuum states between the plates than outside.

- Net force between plates.

- A low-frequency photon travelling in a Casimir vacuum will experience a refractive index $<1$.

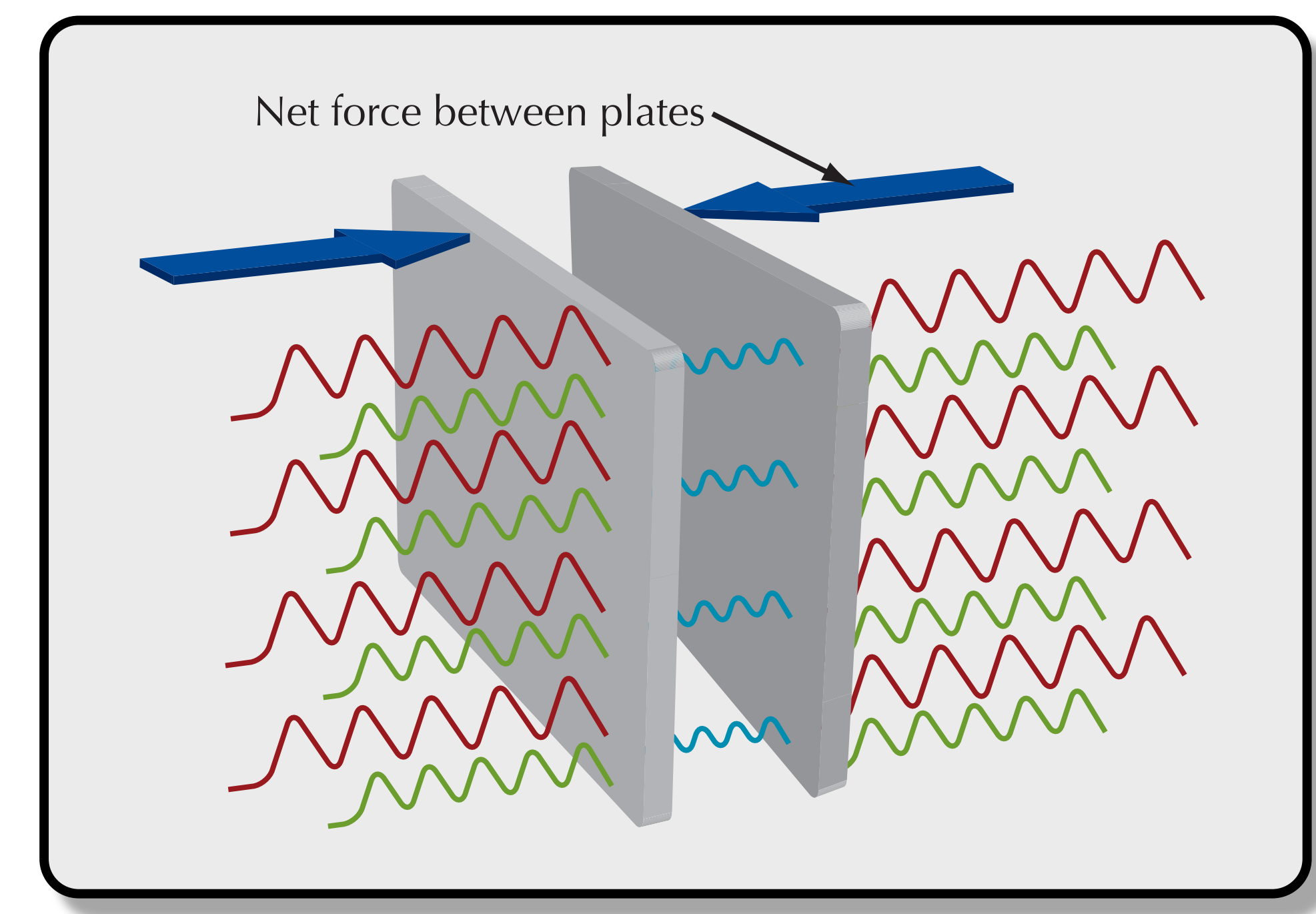

G. Barton, K. Scharnhorst, Journal of Physics A 26, 2037 (1993). (See talks by Gies and van Tiggelen)

- Phase velocity $>1$.

- Group velocity < 1 . 


\section{Pair production}

- High energy photon may create pairs: $\hbar \omega \geq 2 m_{e} c^{2}$

- Multiphoton processes:

- Low-energy photon scatter off electrons, producing high-energy gamma.

- Low-energy photons cause pair production through Sauter-Schwinger mechanism.

- Sauter's resolution to the Klein paradox: static electric field may cause the vacuum to go unstable (Sauter 1931).

- Electrostatic fields under the critical field strength $E_{\text {crit }} \sim 10^{16} \mathrm{~V} / \mathrm{cm}$ is expontially surpressed (Schwinger 1951).

- Relativistic flying/oscillating mirror (Lichters et al., PoP (1996); Bulanov et al, PRL (2003)), relativistic electronic spring (Gonoskov et al., 2011). 


\section{Pair production}

- "Schwinger mechanism" for fields with spatial and temporal variation (see Hebenstreit's talk).

- Temporal compression: increased production rate.

- Spatial compression: lower production rate.

- Laser fields $\longrightarrow$ production rate unknown.

- Schwinger limit: more than necessary (Narozhny et al.).

- Need for theory (e.g. world-line instantons, e.g. Gies, Dunne). 


\section{Pair production}

- "Schwinger mechanism" for fields with spatial and temporal variation (see Hebenstreit's talk).

- Temporal compression: increased production rate.

- Spatial compression: lower production rate.

- Laser fields $\longrightarrow$ production rate unknown.

- Schwinger limit: more than necessary (Narozhny et al.).

- Need for theory (e.g. world-line instantons, e.g. Gies, Dunne).
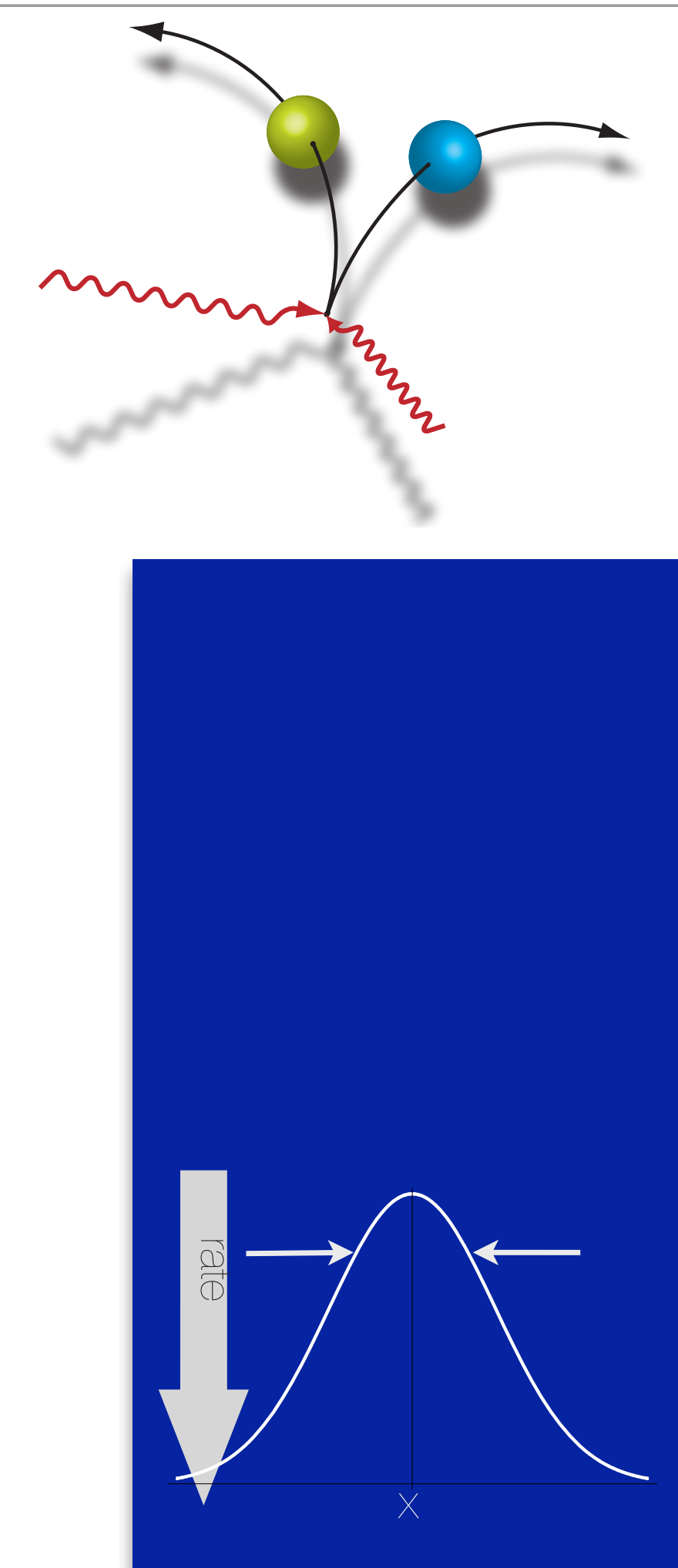


\section{Pair production}

- "Schwinger mechanism" for fields with spatial and temporal variation (see Hebenstreit's talk).

- Temporal compression: increased production rate.

- Spatial compression: lower production rate.

- Laser fields $\longrightarrow$ production rate unknown.

- Schwinger limit: more than necessary (Narozhny et al.).

- Need for theory (e.g. world-line instantons, e.g. Gies, Dunne).
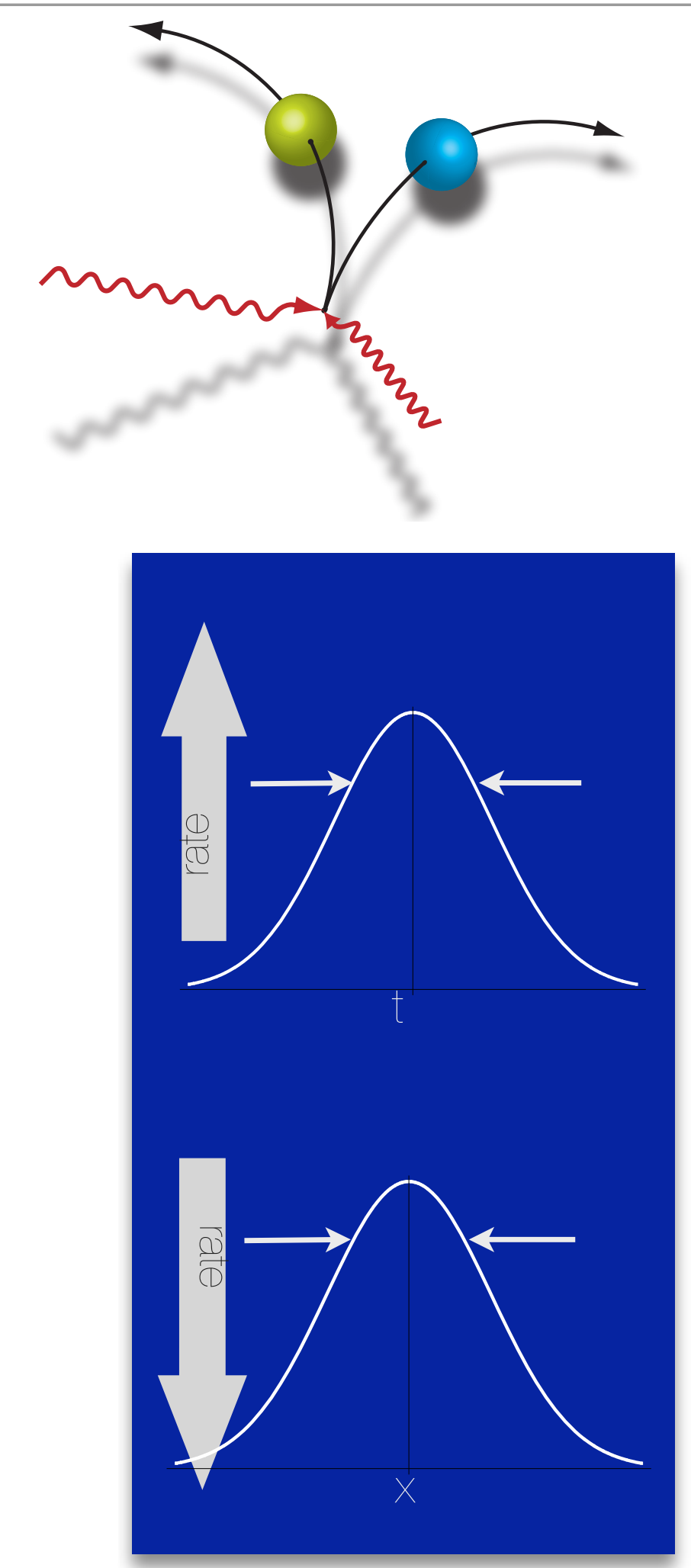


\section{Pair production: stimulated process}

- The SLAC experiment (see also Bula et al., 1996 and Bamber et al., Phys. Rev. D (1999)). Also all-optical.

\section{Boom! From Light Comes Matter}
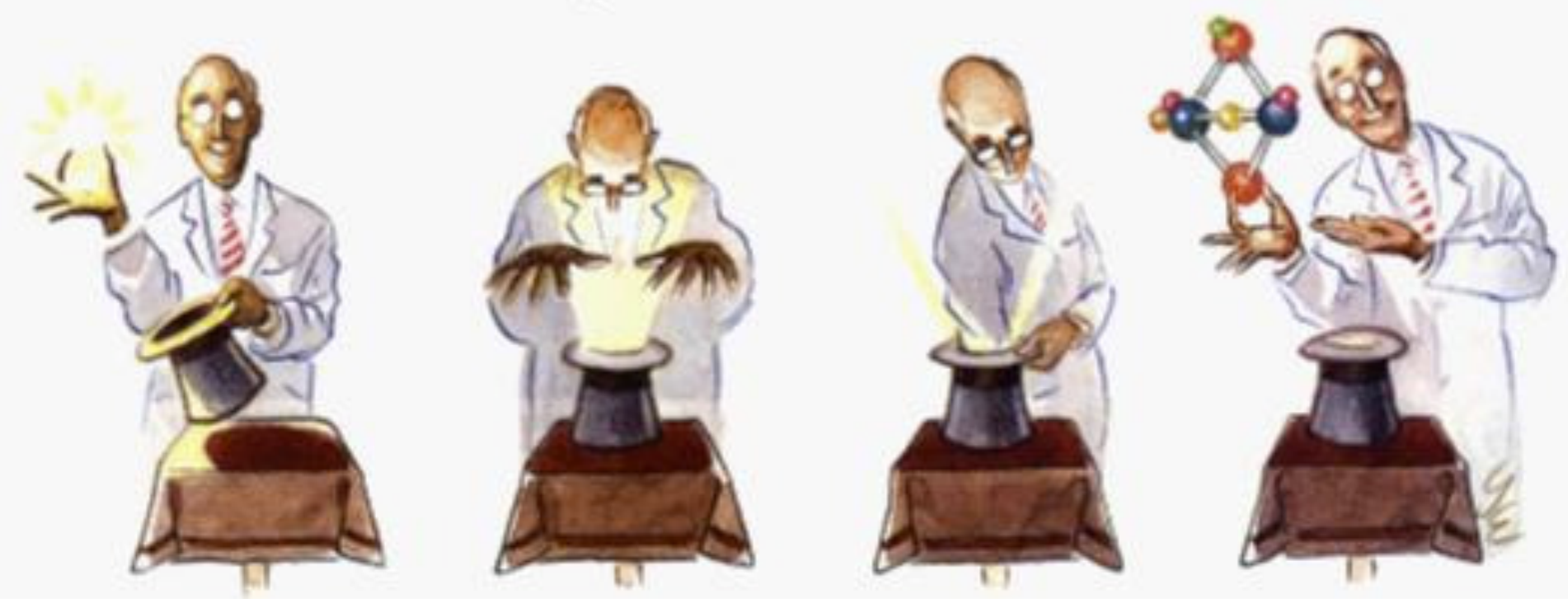

Eisner, Phot. Spectra (1997) 


\section{Pair production: stimulated process}

- The SLAC experiment (see also Bula et al., 1996 and Bamber et al., Phys. Rev. D (1999)). Also all-optical.

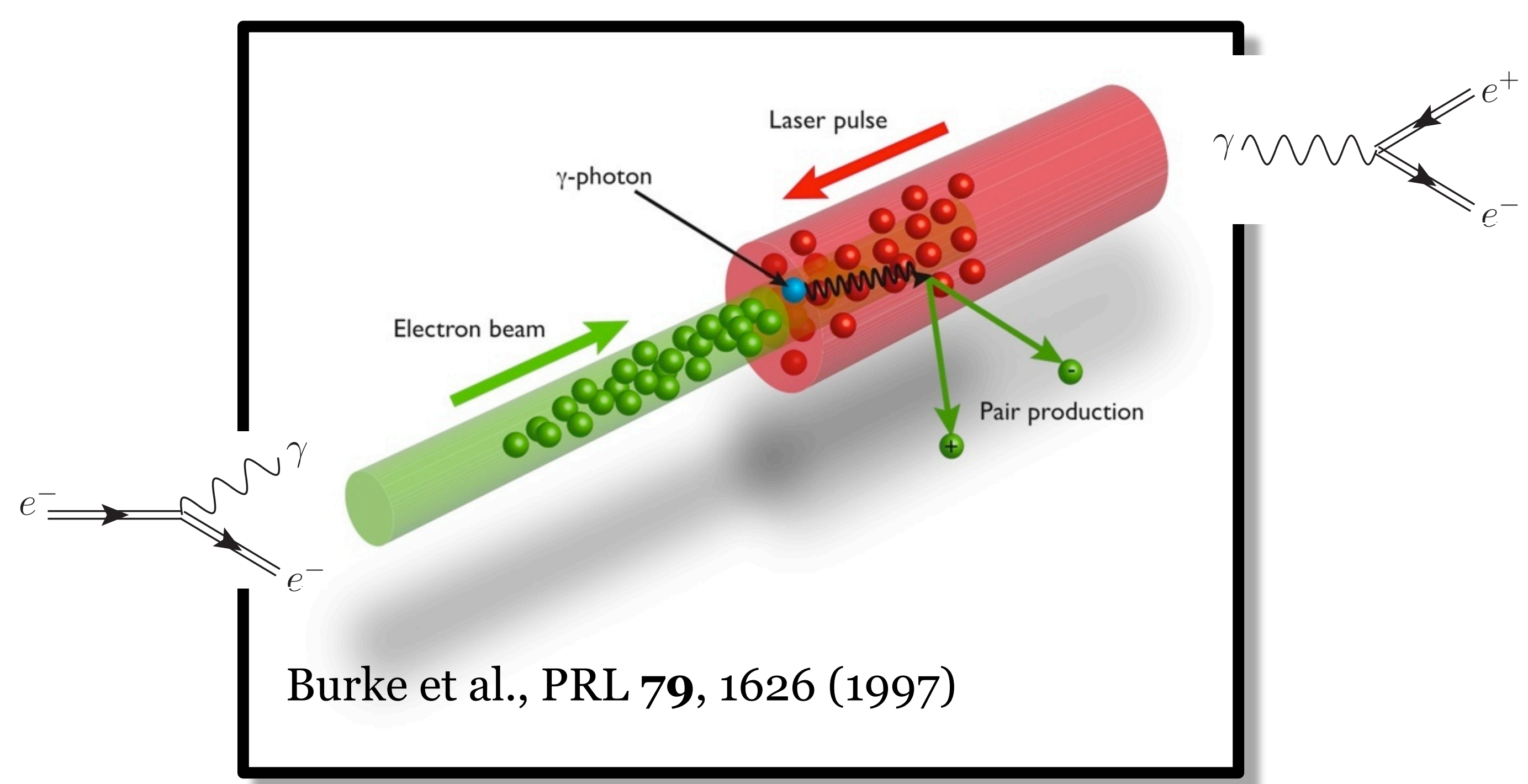

Nonlinear Compton scattering $e+n \omega \rightarrow e^{\prime}+\gamma \longrightarrow\left(\gamma+n \omega \rightarrow e^{+}+e^{-}\right.$ 


\section{Pair production: stimulated process}

- The SLAC experiment (see also Bula et al., 1996 and Bamber et al., Phys. Rev. D (1999)). Also all-optical.

- Experiment reproduced in all-optical setup.

- Spectral properties not detailed enough do actually discern between processes!

- Need stronger fields, better configurations, for better statistics. 


\section{Trident vs. cascading}

- Since the process of producing pairs by different stimulated processes in external fields can put possible upper limits of attainable intensities, it is central to understand what the rates for these processes are.

- As we saw, the cascading process is sequential, and contains only real particles: nonlinear Compton scattering + stimulated pair production.

- The trident process, however, contains a virtual intermediate photon, that by its very definition does not have to live on the shell, i.e., freedom.

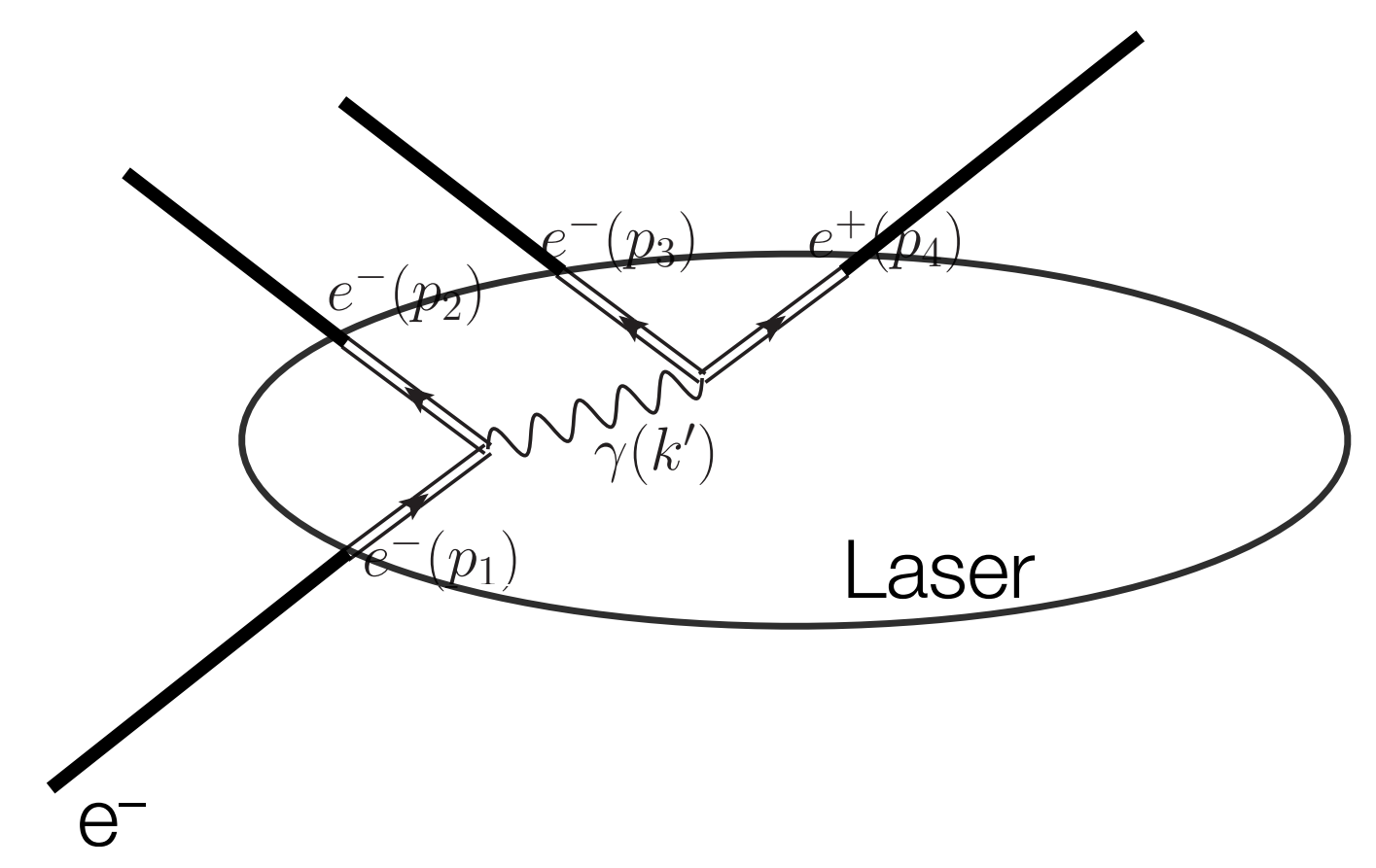

While the cascading photon has to produce the pair inside the pulse, the virtual trident photon must produce a pair, in the pulse or not. What does this imply for the asymptotic state? Can you tell the difference via e.g. a SLAC type of experiment? (Ilderton 2010) 


\section{Finite size effects in pair production}

- Pulse shape dependence in stimulated production (Heinzl et al. 2010).

- The differential cross section plotted as a function of transverse positron momentum for a pulsed plane wave with $4 \mathrm{fs}, 8 \mathrm{fs}$, and 16 fs laser pulses (top to bottom) (Krausz and Ivanov 2009; Mackenroth et al. 2010).

- Longer pulses gives rise to delta-like comb.

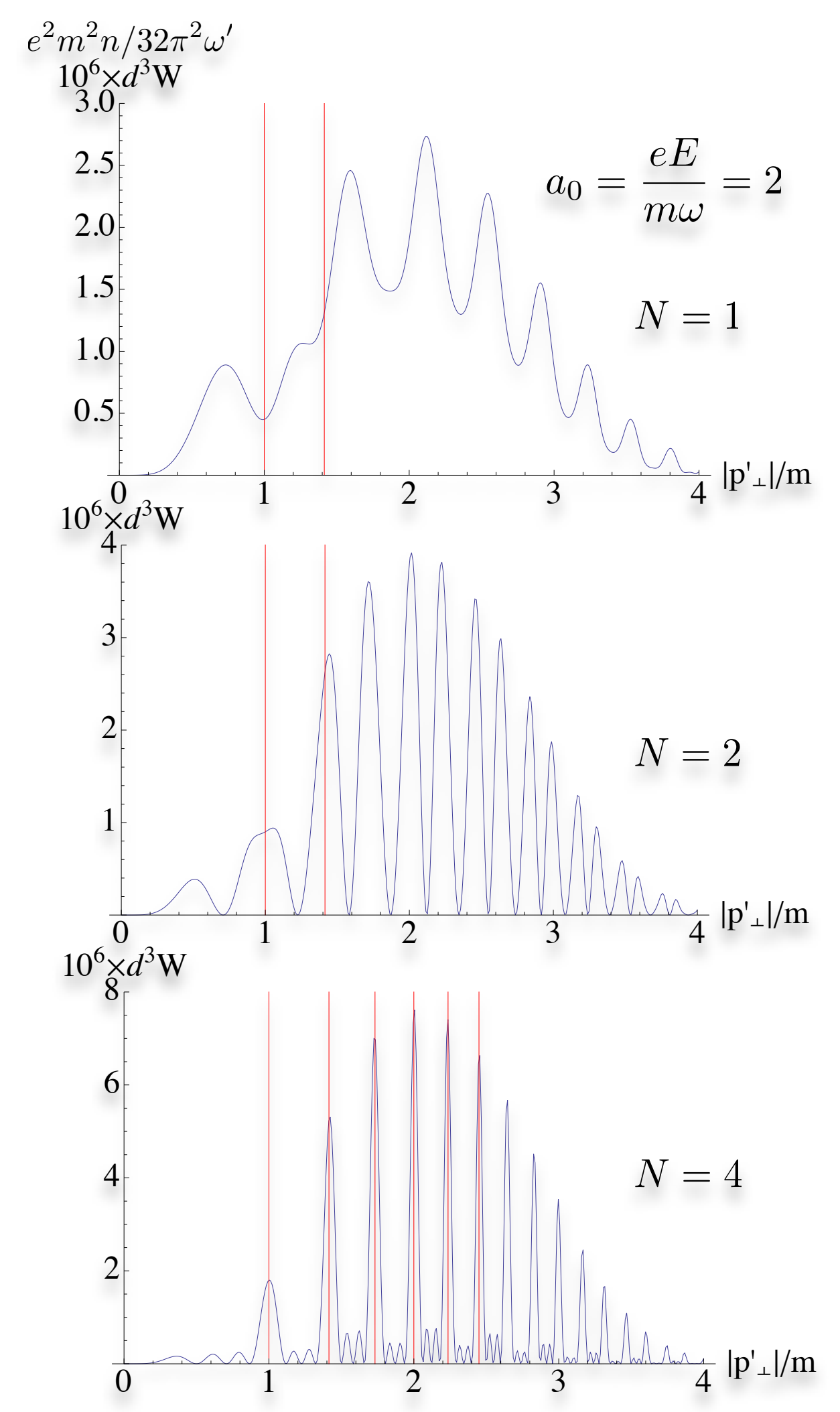

- Finite width gives rate structure and subthreshold peak. 


\section{Pair production: radiation reaction}

- Recent interest in cascading and pair production.

- Previously looked at in astrophysical settings (magnetosphere problems).

- Seemingly conflicting results in the literature.

- Different intensity values for significant cascading to take place.

- Important issue: put constraints on achievable intensities.

- Q1: when is a classical treatment possible? (the transition problem)

- Q2: when in a relativistic quantum regime, how to treat transitions? (the dressing-up problem)

- Q3: when is the division of the pairs into separate $\mathrm{e}^{+}$and $\mathrm{e}^{-}$valid? (the asymptotic problem) 


\section{Radiation reaction/friction}

- Classical radiation reaction described using Lorentz-Abraham-Dirac (LAD) theory

$$
m \dot{u}^{\mu}=e F^{\mu \nu} u_{\nu}-\frac{2}{3} \frac{e^{2}}{4 \pi}\left(u^{\mu} \ddot{u}^{\nu}-u^{\nu} \ddot{u}^{\mu}\right)
$$

- or the perturbative expansion (lacking runaways etc.) due to Landau \& Lifshitz (LL)

$$
\dot{u}^{\mu}=\frac{e}{m} F^{\mu \nu} u_{\nu}+\frac{2}{3} \frac{e^{2}}{4 \pi}\left\{\frac{e}{m^{2}} \dot{F}^{\mu \nu} u_{\nu}+\frac{e^{2}}{m^{3}} F^{\mu \alpha} F_{\alpha}{ }^{\nu} u_{\nu}-\frac{e^{2}}{m^{3}} u_{\alpha} F^{\alpha \nu} F_{\nu}{ }^{\beta} u_{\beta} u^{\mu}\right\}
$$

- Works in classical regime (i.e. current facilities) when $\left(a_{0}=e E / \omega m c\right)$

$$
\chi \equiv \frac{e \hbar \sqrt{\left(F^{\mu \nu} u_{\nu}\right)^{2}}}{m^{2} c^{4}} \ll 1, \quad \Longrightarrow \quad \hbar a_{0} \gamma \omega \ll m c
$$

- QED regime (i.e. next generation regime) when

$$
\chi \sim 1
$$

or when the momentum kick is of the order of the mass of the electron... 


\section{Radiation reaction/friction}

- Why important?

- RR causes emission of high-energy photons.

- The friction gives regimes of electron capture: extended interaction time with the laser (Harvey \& MM, 2011). Note: happens when QED mass-shift important!

- These two in conjunction can yield a significant amount of gammas, and thus pair production via Breit-Wheeler: limits to intensity?

- The transition between classical (LAD/LL) and QED is not well-defined and will significantly affect predictions (Di Piazza et al. 2011, Elkina et al. 2011)

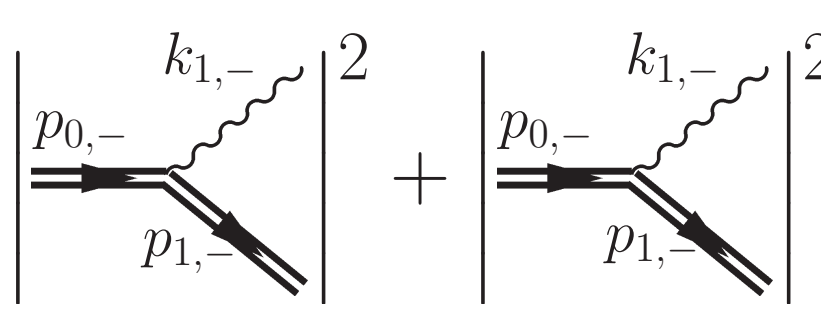

?
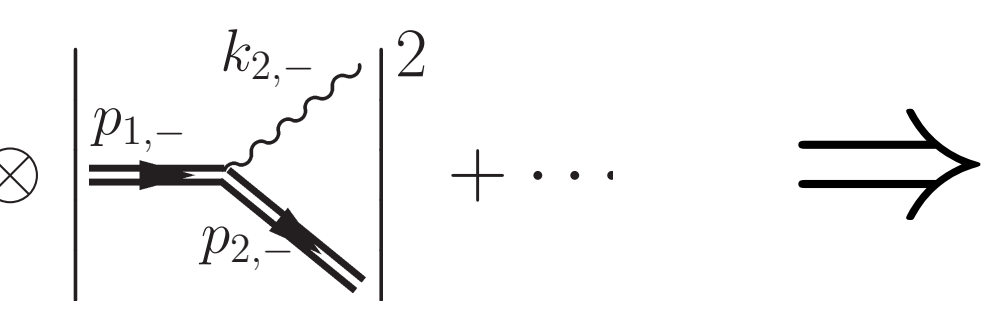

?

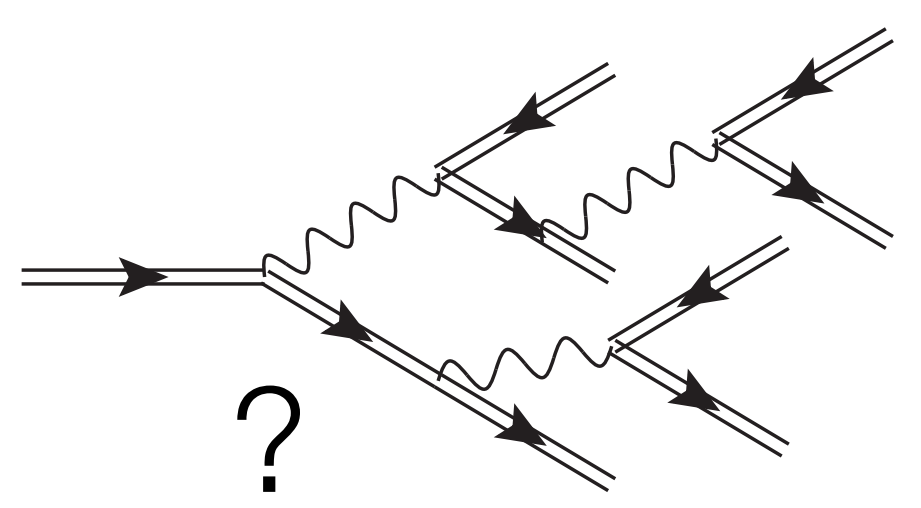




\section{Schwinger-Sauter mechanism}

- Non-perturbative effect.
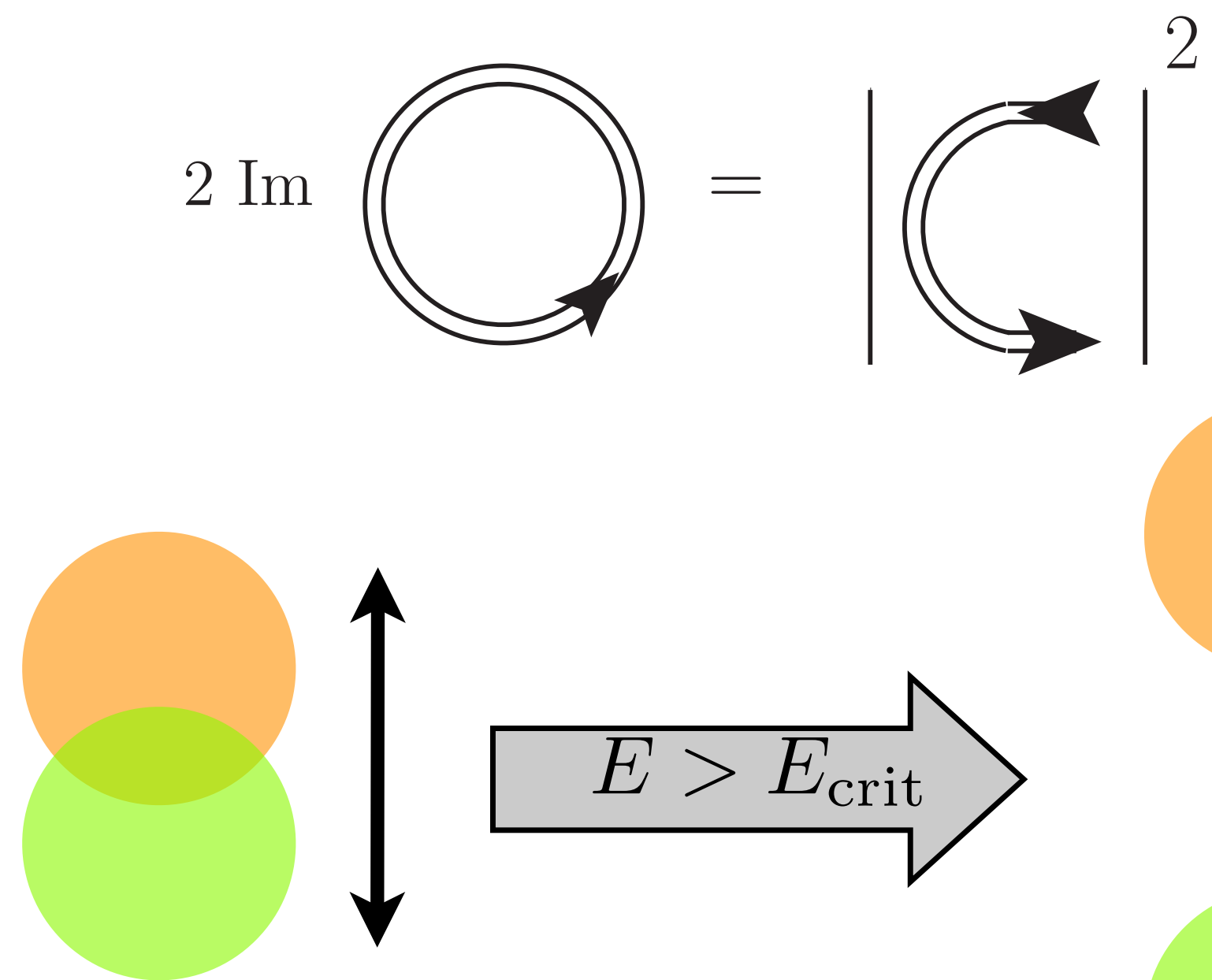

Separating field $E$

- For general field, we expect pair creation for invariant

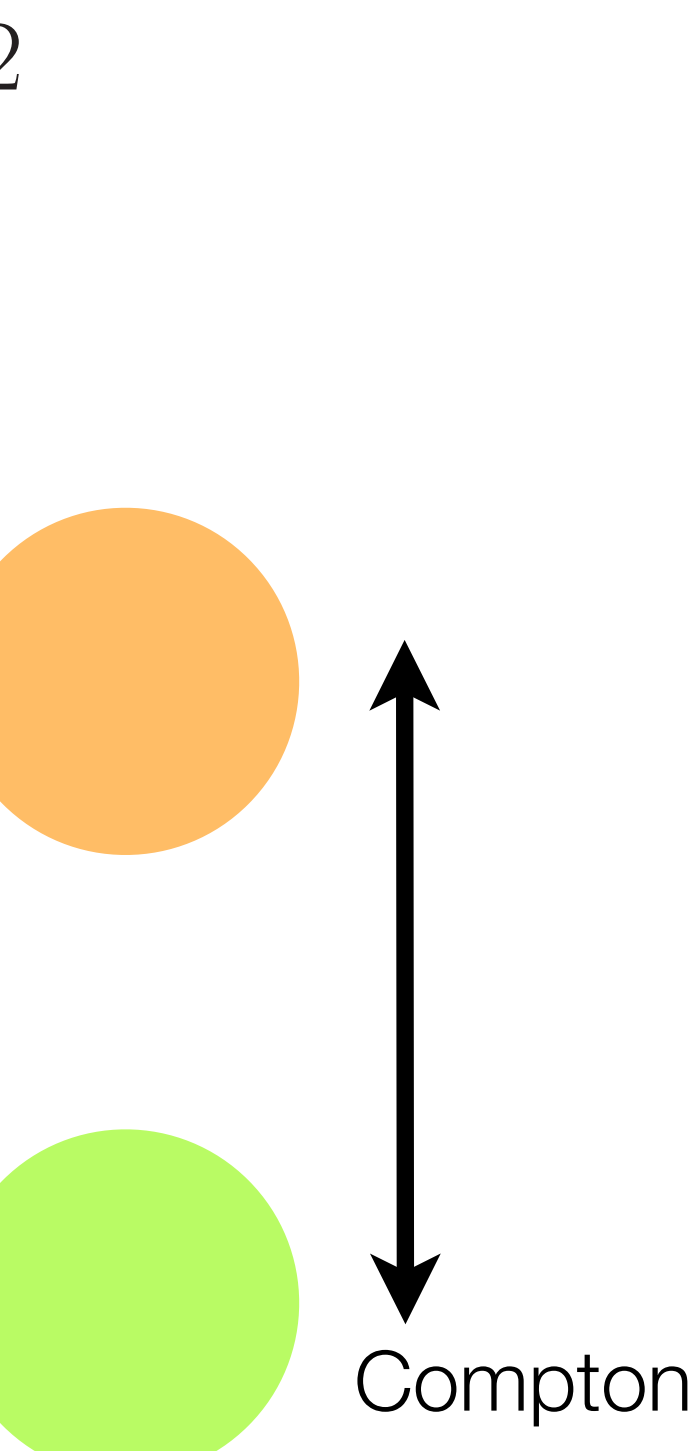

$$
E^{2}-B^{2}>E_{\text {crit }}^{2}
$$




\section{Schwinger-Sauter mechanism: plasmas}

- For oscillating fields $B \sim \frac{k}{\omega} E$

- Thus, $E^{2}-B^{2}=\left(1-\frac{k^{2}}{\omega^{2}}\right) E^{2}$, which is zero for plane waves.

- However, in a plasma $\omega^{2}=k^{2}+\omega_{p}^{2}$

- Thus, we should expect that the pair production fields should satisfy

$$
\frac{\omega_{p}}{\omega} E>E_{\text {crit }}
$$

- The generation of a pair plasma by the Schwinger mechanism affects the production rate (Bulanov 2004).

- Plasma assisted pair production? 


\section{Schwinger-Sauter mechanism: quantum kinetics}

- Use quantum kinetic theory to investigate non-perturbative pair production.

- Simplest form, homogeneous case $\frac{\partial f(\mathbf{p}, t)}{\partial t}=\left(\frac{d f}{d t}\right)_{\text {Schwinger }}$

- Powerful technique with close analogue to measurements and classical theory. Suitable for numerics.

- Distribution function tells how the pair density evolves in phase space.

- Electric field $E(t)$ (Alkoher et al. 2001), explicit production term.

- For XFEL data (strong focusing, diffraction limit, $E=0.1 E_{\text {crit), each pulse }}$ would produce 1000 pairs. 


\section{Schwinger-Sauter mechanism: quantum kinetics}

- Taking the full structure of QED into account: non-scalar distribution function.

- So called Dirac-Heisenberg-Wigner formalism.

$$
\frac{\partial f(\mathbf{p}, t)}{\partial t}=\left(\frac{d f}{d t}\right)_{\text {Schwinger }}\left[\frac{\partial}{\partial t}+\Delta(x, p, t)\right] \underbrace{\mathcal{W}(x, p, t)=\text { stuff }}_{\begin{array}{c}
\text { Nonlocal differential operator, } \\
\text { depends on field } E(x, t)
\end{array}}
$$

- Gives particle self-bunching (nonlinear effect) in Schwinger pair production. 


\section{Pair production: the fight against exponential supression}

- Using pre-factor [laser four-colume/Compton four-volume $\approx 10^{24}$ ] to increase pair production rate (Narozhny et al., 2004).

- Superimposed oscillatory fields (substructure) gives assisted pair production (Dunne, Gies, Schützhold, 2008, 2009).

- $E>>B$ for counterpropagating/standing waves (Gregori et al., Astra Gemini/ RAL experiment, 2010).

- XFEL-optical combos (Hebenstreit, Ilderton, MM 2011).

- Complex beam configurations (Bulanov et al., 2010). Vacuum fluctuations

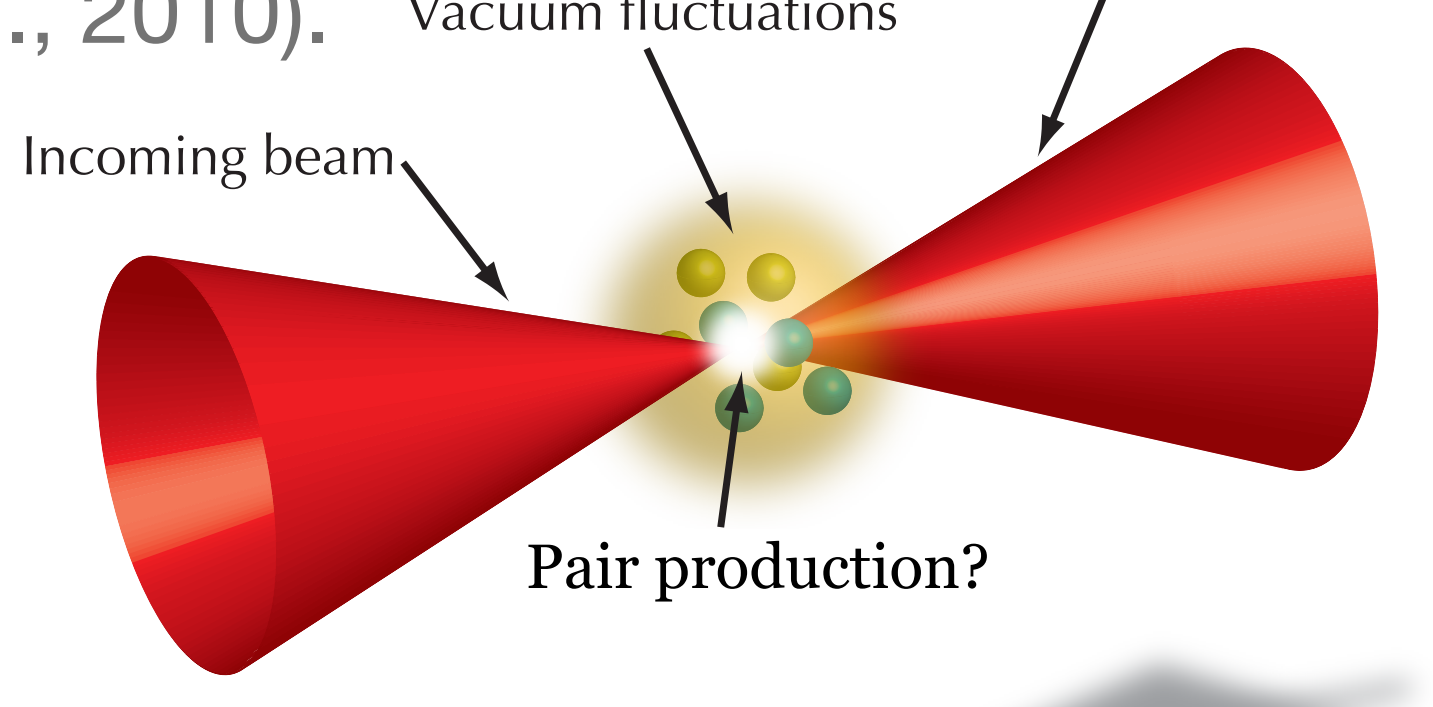




\section{Pair production: importance}

- Nonperturbative quantum field theory: truly relativistic quantum field theory.

- Techniques developed for QED pair production useful for QFT in general: kinetic simulations (e.g. Ruhl), quantum kinetic developments (e.g. Alkhofer, Hebenstreit), world-line and lightcone techniques (Gies, Dunne, Heinzl, Ilderton).

- Similarities to strong field ionization problems (Reiss, PRL 2008; Blaga et al., Nature Phys. 2009).

- Nonlinear scattering events (Heinzl et al., PRA 2010).

- Source of ep-plasma? 


\section{Discerning between processes?}

- There are a large number of possible processes that can generate pairs.

- Another example: production of fraction of superthermal electrons in lasers

$$
E \sim\left[\left(1+\frac{\alpha I \lambda^{2}}{I_{\mathrm{crit}} \lambda_{e}^{2}}\right)^{1 / 2}-1\right] m c^{2}=\left[\left(1+\alpha a_{0}^{2}\right)^{1 / 2}-1\right] m c^{2}
$$

- $a_{0}>\sqrt{3 / \alpha}$ gives electron energy above rest mass. Can contribute to pair production.

- How to discern between trident, stimulated pair production (cascades) and bremsstrahlung generation, Schwinger mechanism?

- Look at spectral properties of emissions, possible to find unique features?

- Possible to set up experiments that can deal with this? 


\section{Pair production: theoretical developments.}

Pair production one aspect of a more complex computational problem:

how to do nonperturbative many-body

quantum physics?

Difficult and necessary computational developments. 


\section{Pair production: theoretical developments.}

Pair production one aspect of a more complex computational problem: how to do nonperturbative many-body quantum physics?

Difficult and necessary computational developments.

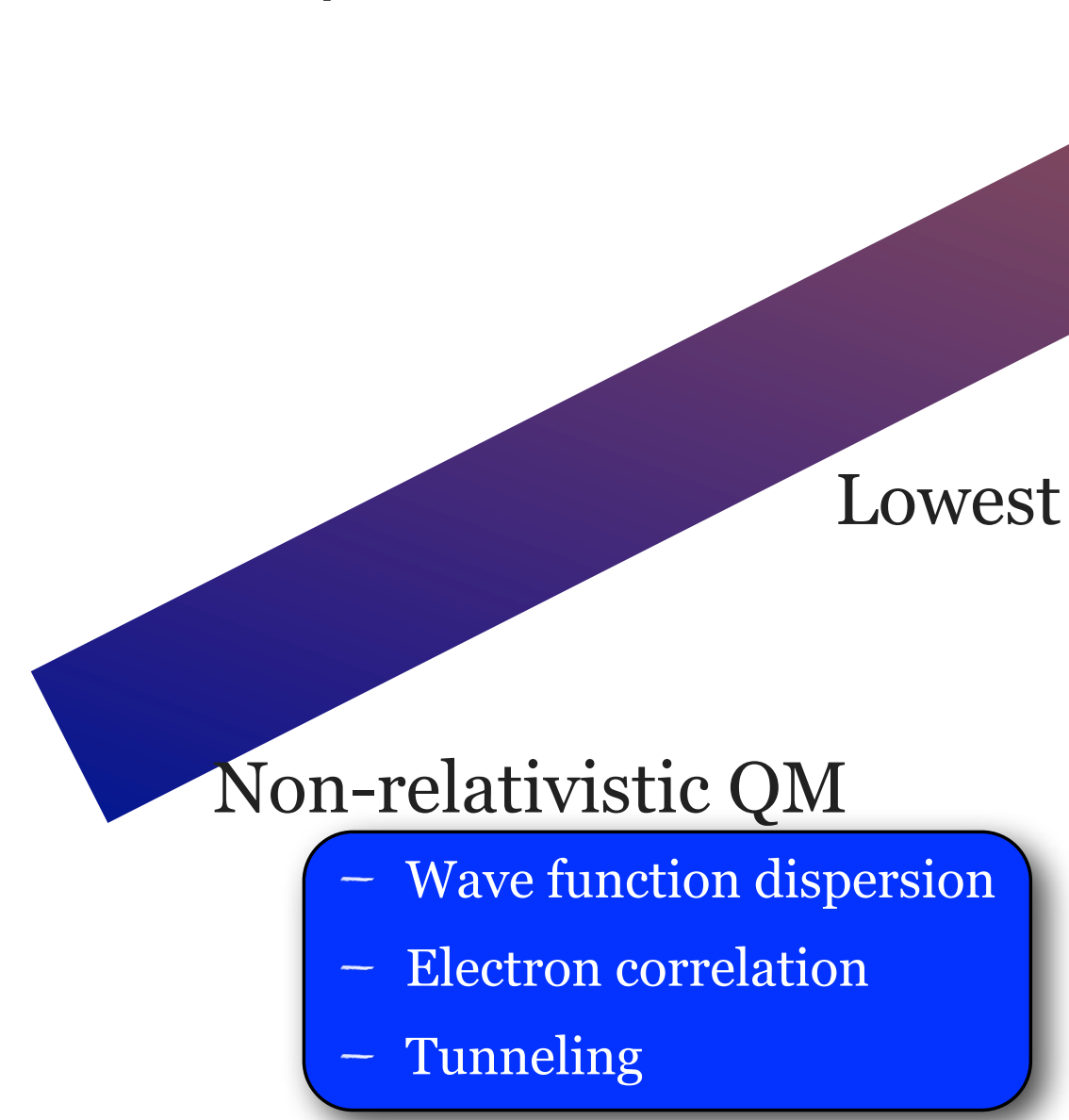

Relativistic QM

- Fermi-Dirac statistics

- Zitterbewegung

order relativistic QM

- Magnetization

- Spin-orbit coupling

Classical/quantum theory of radiation reaction? 


\section{Particle-in-cell simulations}

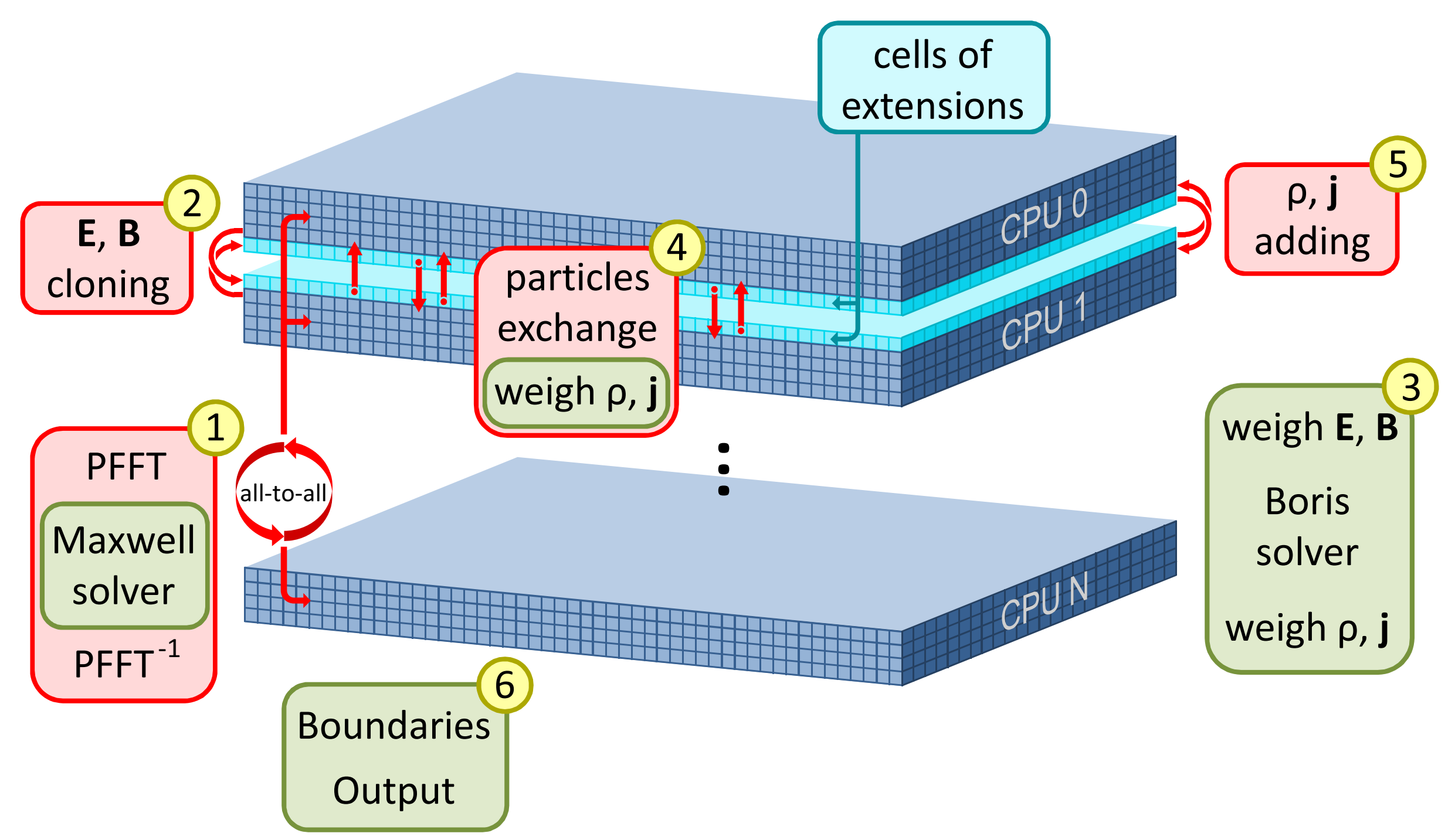




\section{Particle-in-cell simulations}

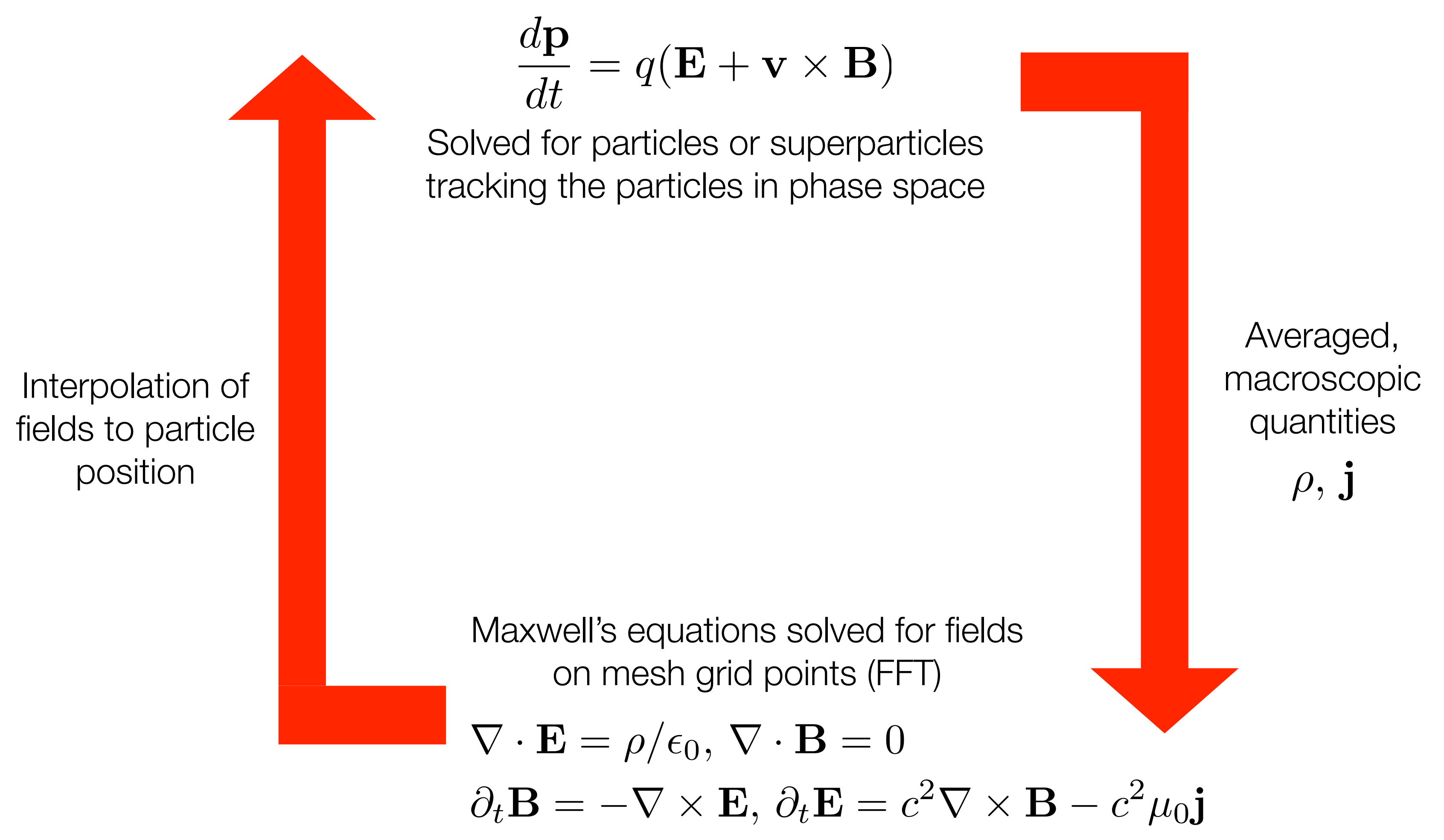




\section{Particle-in-cell simulations}

- How do we include quantum processes?

- Classical PIC simulations: tens of billions of particles; photons are fields.

- Proper photons: laser pulse has approximately a hundred billion billion photons!

- Not possible to include a quantum treatment of all photons.

- Use division into classical field (large number of photons) and quantum (few photons).

- Problem: double counting!

- Nonlinear Compton scattering: classical limit = Lorentz force

- Contains higher order quantum corrections to Lorentz force.

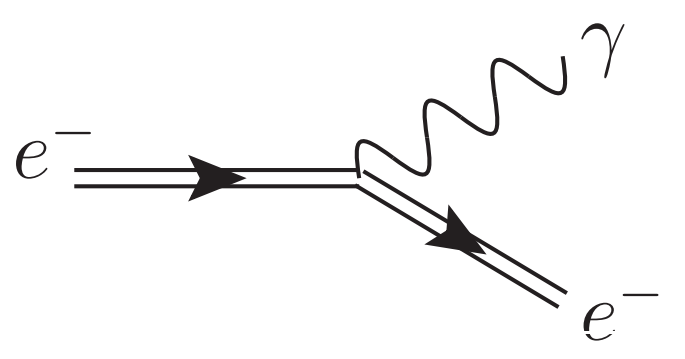

- How to deal with radiation reaction (non-perturbative, not Landau-Lifshitz)? 


\section{The Unruh effect}

- A touch of gravitational physics using lasers?

- Hawking (1974) and Unruh (1976): deep connection between thermodynamics, field theory, and gravity.
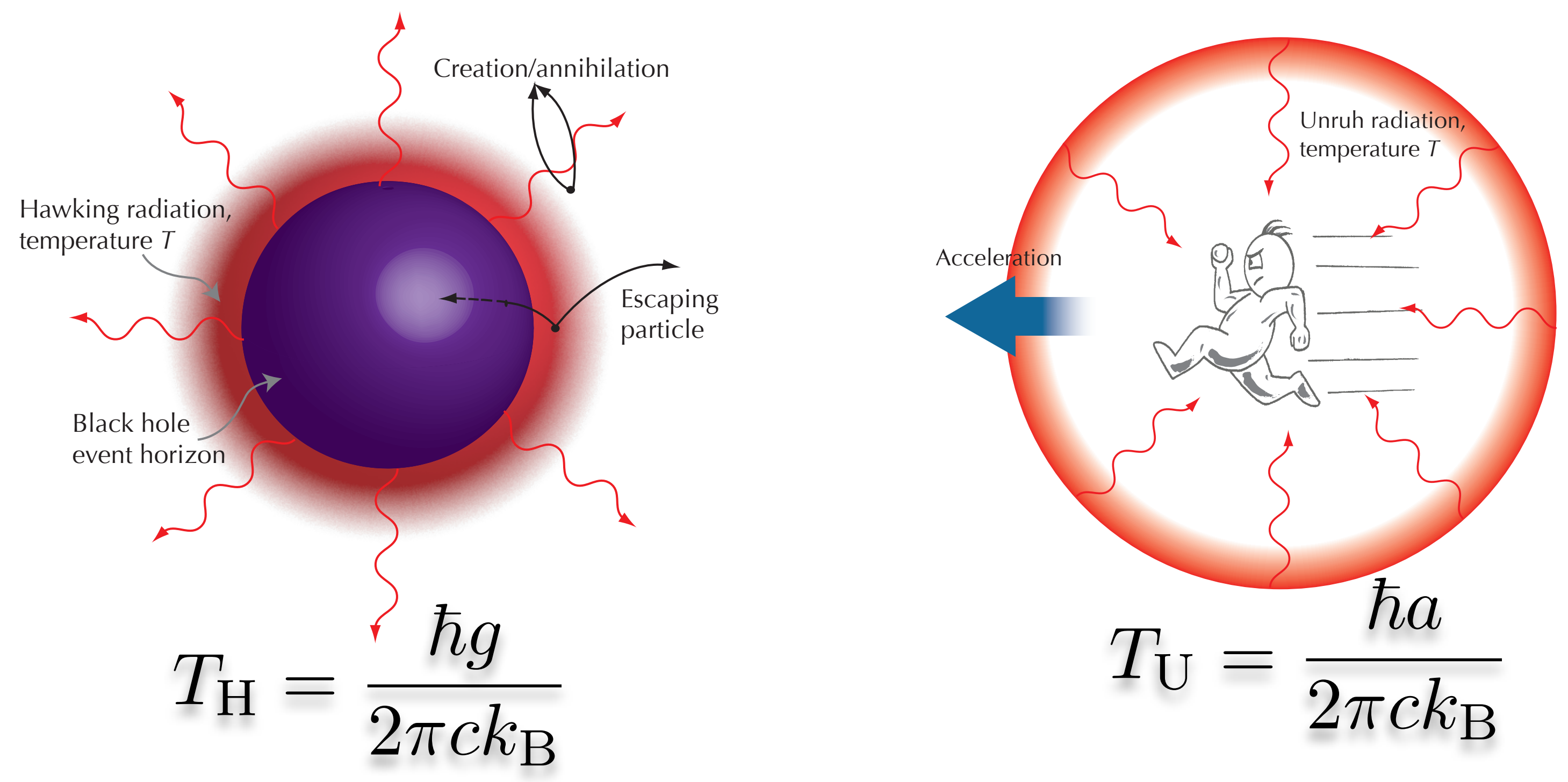


\section{The Unruh effect}

- A touch of gravitational physics using lasers?

- Hawking (1974) and Unruh (1976): deep connection between thermodynamics, field theory, and gravity.

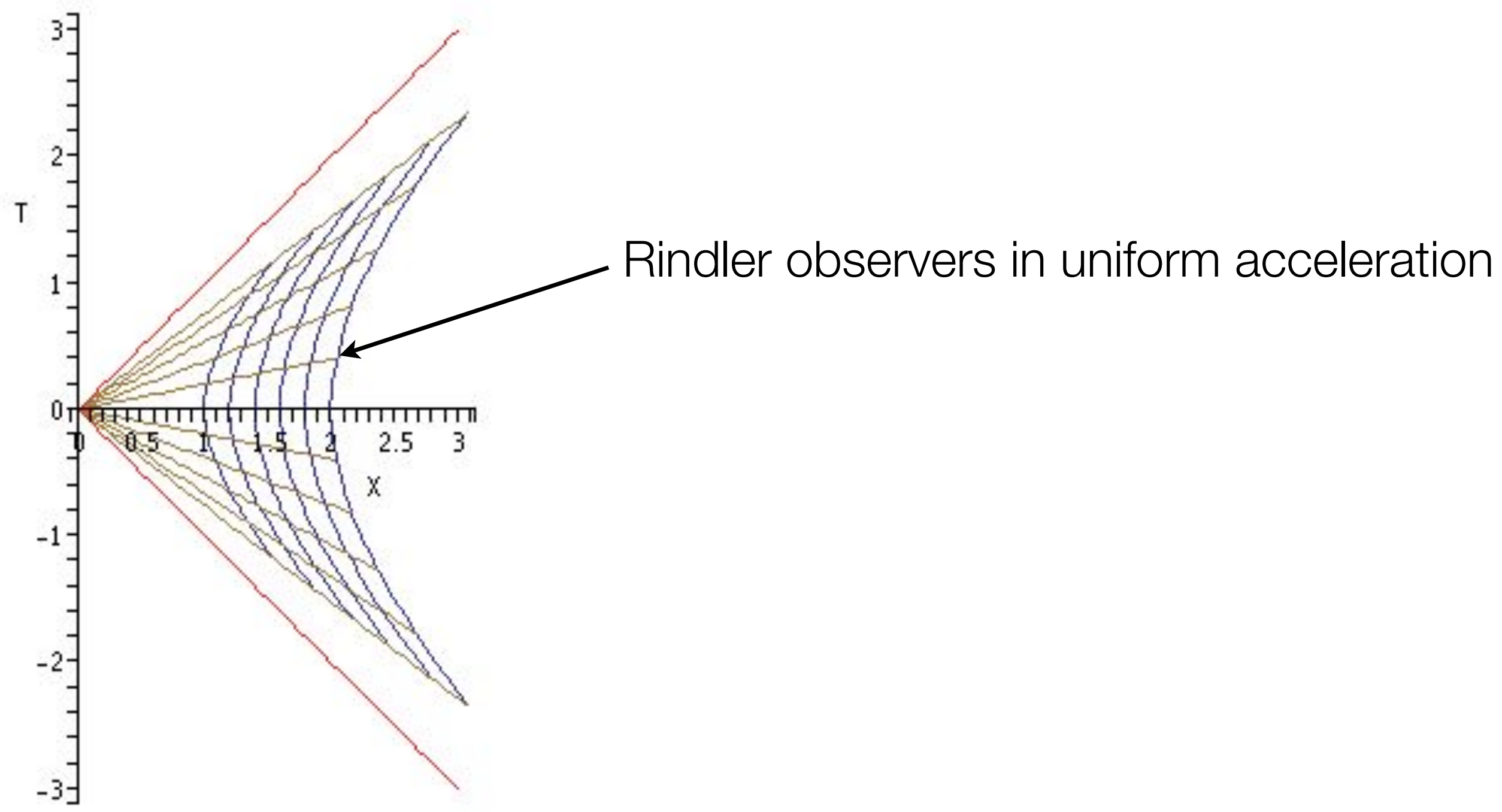




\section{The Unruh effect}

- A touch of gravitational physics using lasers?

- Hawking (1974) and Unruh (1976): deep connection between thermodynamics, field theory, and gravity.
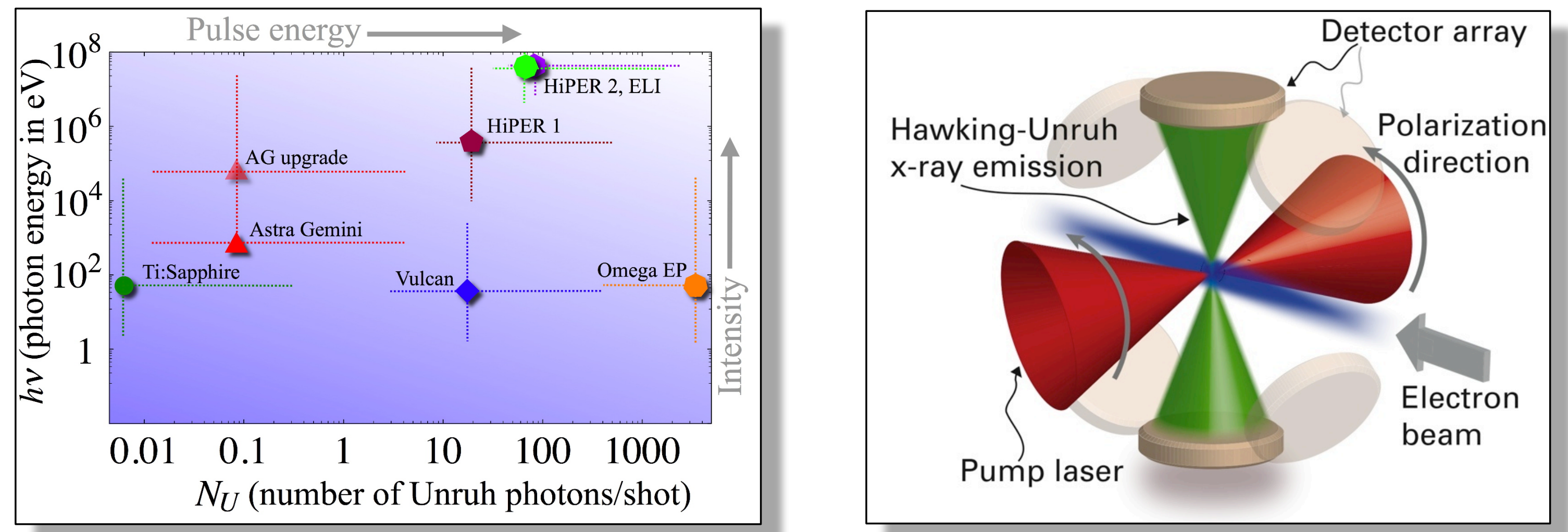

- Experiments (Chen \&Tajima 1999; Schützhold et al. 2006; Brodin et al. 2008). (See also dynamical Casimir, van Tiggelen) 


\section{Exotic physics?}

- Probing of spacetime structure?

- Noncommutativity (NC) between spacetime coords infered from quantum gravity/string schemes; IR/ UV mixing (Amelino-Camelia et al. 2005).

- Noncommuting coordinates implies position uncertainty which eliminates short-distance singularities.

- Analogue: in the plane othogonal to a very strong magnetic field we have coordinate NC (lowest Landau level) [Jackiw, Ann. Henri Poincare (2003)].
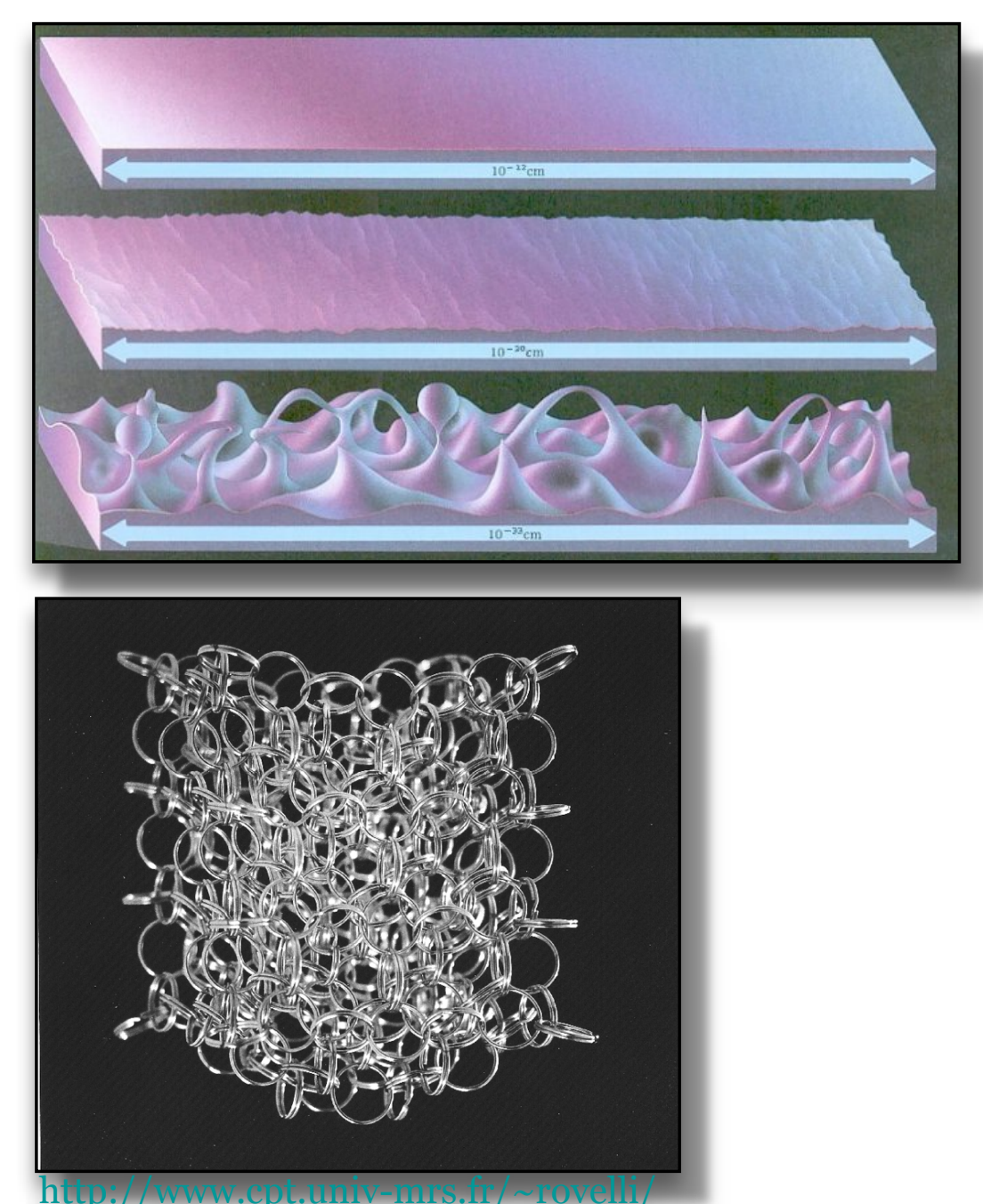

- Suggested to be probed using vacuum birefringence experiments (Abel et al. JHEP 2006). 


\section{Noncommutative spacetimes}

- Regular quantum mechanics

$$
\left[x_{a}, v_{b}\right]=\frac{i \hbar}{m} \delta_{a b} \quad\left[x_{a}, x_{b}\right]=0
$$

- Particle in weak magnetic field

$$
\left[v_{a}, v_{b}\right]=i \hbar \frac{q}{m^{2} c} \epsilon_{a b c} B_{c}
$$

- Particle in strong magnetic field $(m \rightarrow 0) \quad\left[x_{a}, x_{b}\right]=\frac{i \hbar}{q B} \epsilon_{a b}$

- Quantum gravity schemes, emergent quantum mechanics. $\left[x_{\mu}, x_{\nu}\right]=i \hbar \theta_{\mu \nu}$ 


\section{Multi-photon NC physics?}

- Does probing NC physics require reaching Planck scale?

- Quantum foam dispersion through e.g. accumulative gamma-ray effects [Ellis \& Amelino-Camelia]; not first principles!

$$
p^{2}=E^{2}\left[1+f\left(E / E_{\mathrm{QG}}\right)\right]
$$

- Instead of high energy: low energy, high intensity.

- Big photon numbers in lasers + ultra-relativistic beam facilities.

*Multi-photon probes of NC?

*0909.0656 [hep-ph] and 0908.2917 [hep-ph].

* Strong field QED and NCQED processes in intense laser beams. 


\section{Noncommutativity: NLC}

\section{Forbidden by momentum conservation in vacuum in QED/NCQED.}

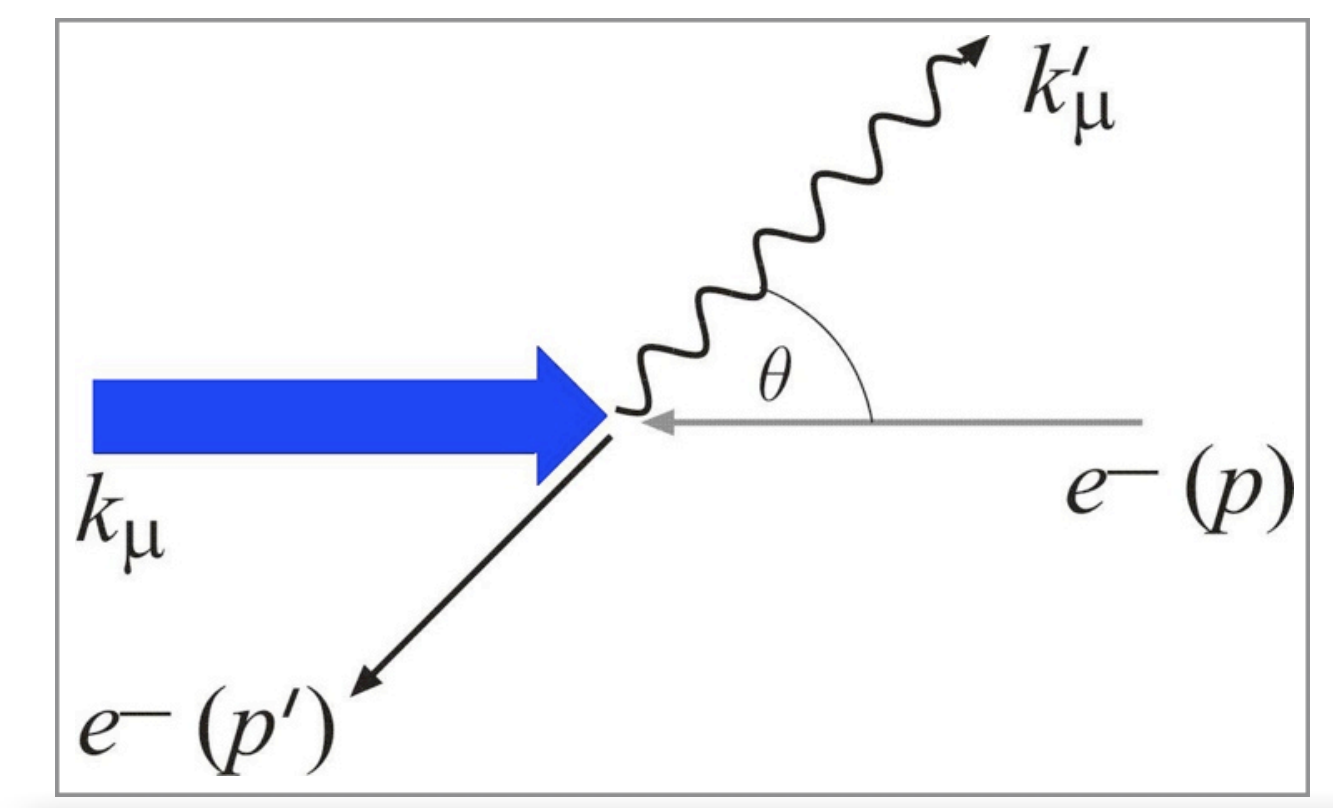

- Nonlinear Compton: many photons.

- $n=1, \ldots, \infty$ processes

- High intensity, $a_{0}=(\mathrm{m} / \mathrm{\omega})\left(E / E_{\text {crit }}\right)>1$ [optical regime $v^{-1}=m / \omega \approx 10^{6} ; E / E_{\text {crit }} \approx 10^{-4}$ (current) $10^{-2}$ (future)].

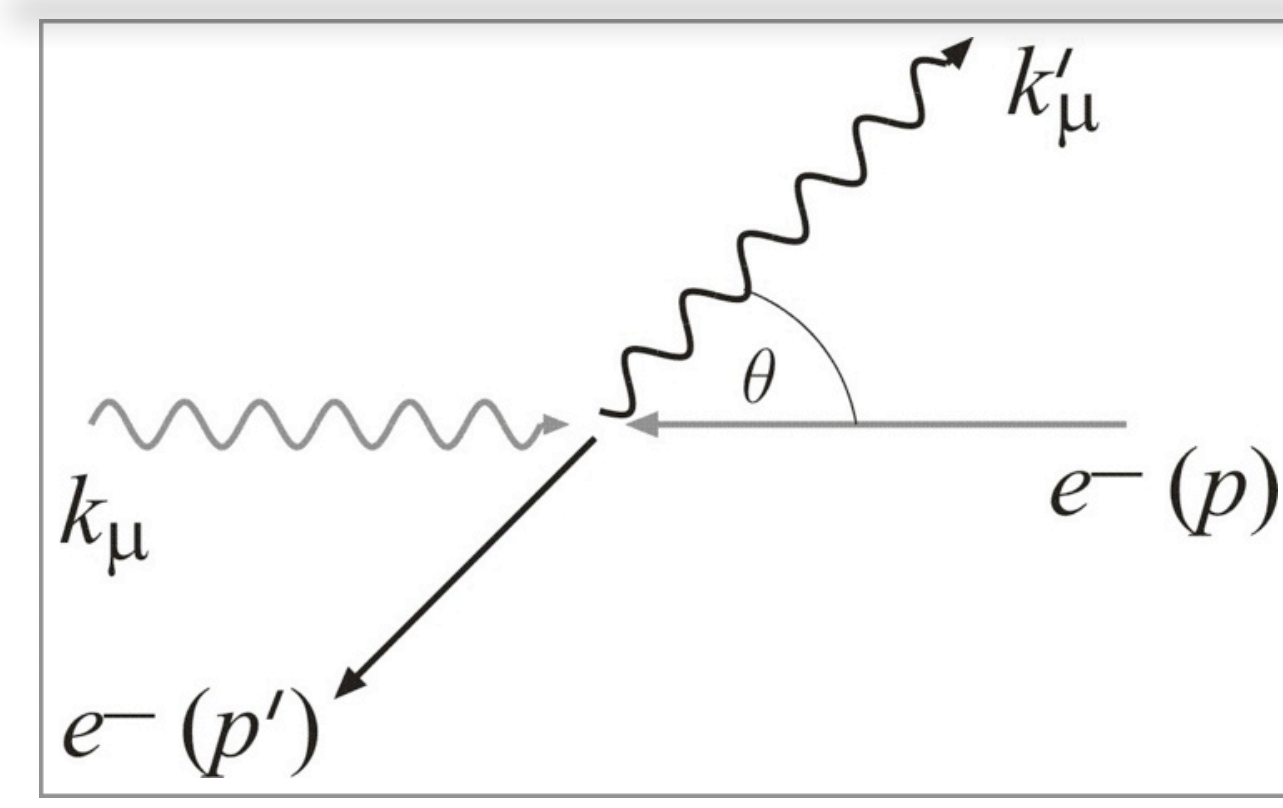

- Ordinary Compton: one photon

- $n=1$.

- Low intensity, $a_{0} \approx 0$. 


\section{Noncommutativity: NLC}
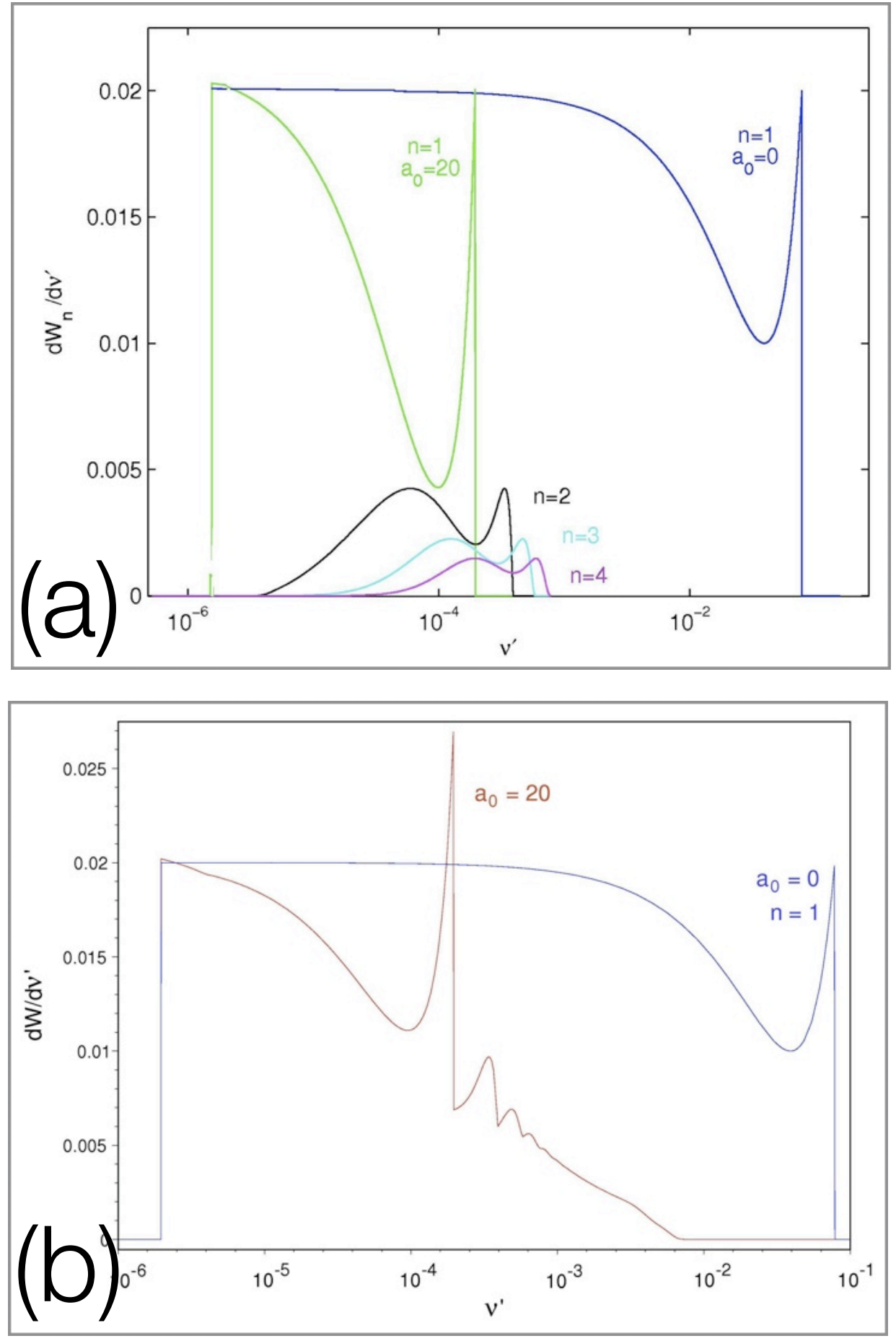

- Cross section comparisons (see 0903.4151 [hep-ph)] for Compton in QED.

- Cross section for $40 \mathrm{MeV}$ electrons and optical photons.

- (a) gives cross section for single wavelengths; "pile up" of cross section for $n>1$.

- (b) gives sum over 50 first harmonics; nonlinear Compton edge dominates.

Compton

$\omega \rightarrow 4 \gamma^{2} \omega$
Nonlinear Compton

$$
\omega \rightarrow \frac{4 \gamma^{2} \omega}{1+a_{o}^{2}}
$$




\section{Noncommutativity: NLC}

-What happens in NCQED?

$$
\left[x_{\mu}, x_{\nu}\right]=i \hbar \theta_{\mu \nu} \quad \theta_{\mu \nu}=c_{\mu \nu} / \hbar \Lambda^{2} \quad\left|c_{\mu \nu}\right| \sim 1 \quad \Lambda \sim 1 \mathrm{TeV} ?
$$

- Photons are self-interacting in NCQED; photon dressed by background field (as a fermion would).

- Photons see a phase shifted background field

$$
a_{\mu}\left(k . x+\hbar k_{\mu} \theta^{\mu \nu} k_{\nu}^{\prime} / 2\right)-a_{\mu}\left(k . x-\hbar k_{\mu} \theta^{\mu \nu} k_{\nu}^{\prime} / 2\right)
$$

- "Extended" photon; dressed photon acquires effective mass.

$$
m_{\mathrm{eff}}=2 m a_{0} \sin \left(k_{\mu} \theta^{\mu \nu} k_{\nu}^{\prime} / 2\right)
$$




\section{Noncommutativity: NLC}

-All NCQED effects comes from effective photon mass.

- Need to maximize effective mass.

- $k$ ' is function of $a_{0}$, not independently tunable to the incoming laser photons.

- Thus, cranking up the laser intensity will saturate the mass at $10^{-11}$ of the electron mass, at most.

- However, for the inverse process, multi-photon pair production, $k^{\prime}$ independent of $a_{0}$. Tunable. 


\section{Noncommutatitivy: Pair production}

- Multi-photon pair production:

$$
n \gamma+\gamma^{\prime} \rightarrow e^{-}+e^{+}
$$

- Threshold number (of laser photons) changes:

$$
n_{0} \equiv \frac{2 m^{2}\left(1+a_{0}^{2}\right)}{k . k^{\prime}} \Rightarrow n_{0, \theta} \equiv \frac{2 m^{2}\left[1+a_{0}^{2}-a_{0}^{2} \sin ^{2}\left(k_{\mu} \theta^{\mu \nu} k_{\nu}^{\prime} / 2\right)\right]}{k . k^{\prime}}
$$

- Threshold is reduced in NCQED.

- Moreover, NC breaks azimuthal symmetry around beam axis.

- Oscillatory dependence of cross section on azimuthal angle:

$$
d \sigma / d \phi=f(\phi)
$$




\section{Pair production}

Plot of $d \sigma / d \phi=f(\phi)$ normalized by the QED value (no azimuthal dependence).

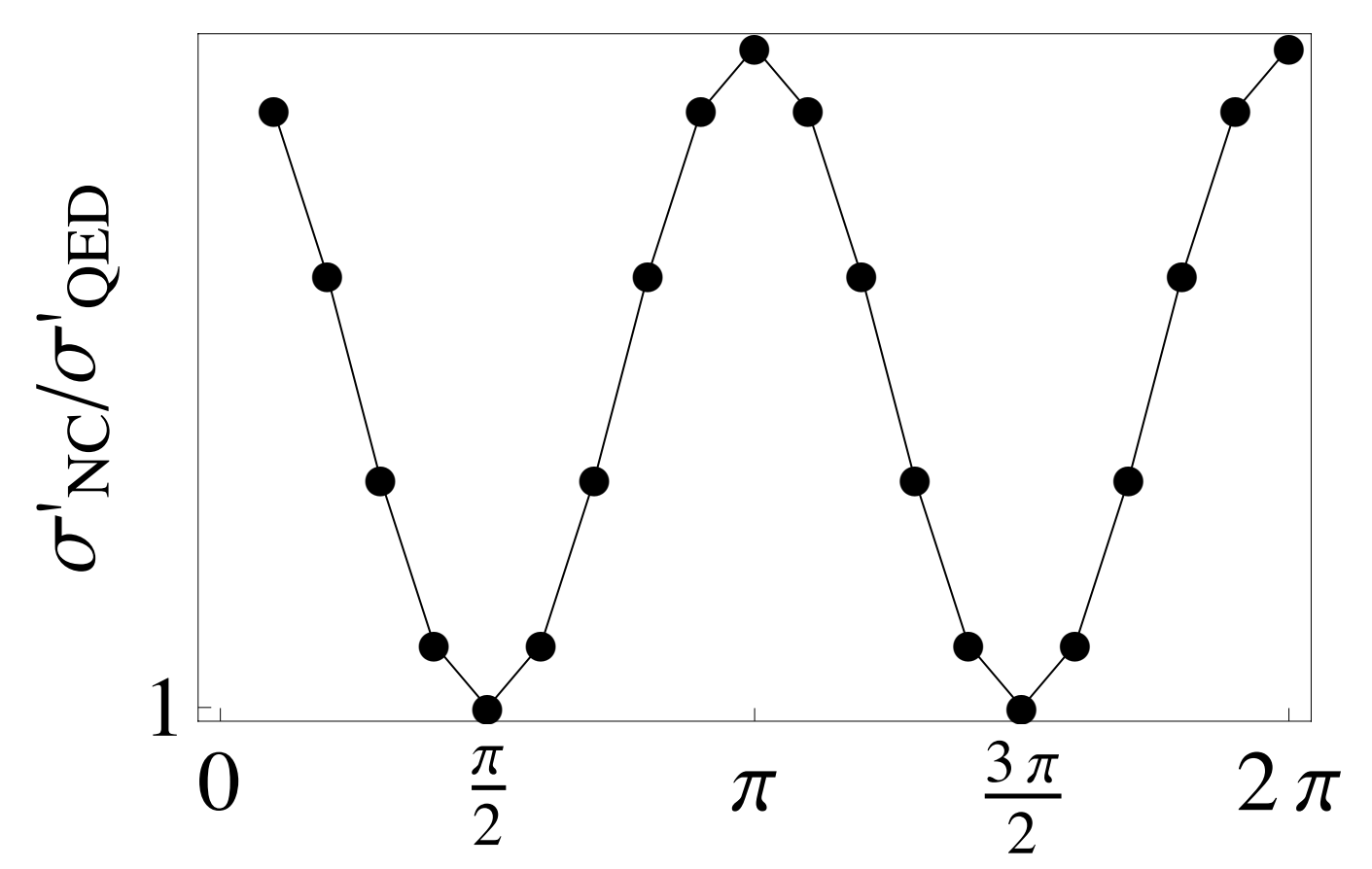

Setup: create high-energy photon $\gamma^{\phi}$ via Compton backscattering in FEL. Collide $\gamma^{\prime}$ with $n y$ from laser. For ELI values

$$
a_{0} \sim 10^{3}, \quad a_{0} \sin \left(k_{\mu} \theta^{\mu \nu} k_{\nu}^{\prime} / 2\right) \sim 10^{-3}, n_{0, \theta}=O(1)
$$




\section{Exotic physics?}

- Noncommuting coordinates $\left[x^{\mu}, x^{\nu}\right]=i \Theta^{\mu \nu}$

- Laser intensity effects to counter the energy scale (Heinzl et al., PRD 2010).

- Pair production:

- depends periodically on collision angle,

- larger cross section,

- threshold (number of photons, for ELI parameters) lowered from QED value.

$$
n_{0, \Theta} \approx n_{0}-\frac{2 \times 10^{8} m^{6}}{k \cdot k^{\prime}}|\Theta|^{2}
$$

- Laser can thus put lower limits on the involved phenomenological parameters. 


\section{Exotic physics? Possible routes for detection.}

Effects through parametrized generalized Maxwell-Dirac system (Lämmerzahl, Appl. Phys. B, 2006)

- Birefringence.

- Anisotropic speed of light.

- Anisotropy in quantum fields.

- Violations of universality of free fall and the universality of the gravitational redshift.

- Time and space variations of "constants".

- Charge non-conservations.

- Anomalous dispersion.

- Decoherence and spacetime fluctuations.

- Modified interference.

- Non-localities. 


\section{Conclusions}

- Ample opportunities for probing new physics with high-power laser.

- Requires a strong collaboration between theory, simulations, and experiments.

- In particular, still many parts of QED that are not computationally viable, or that need independent verifications.

- The classical-quantum transition of radiation reaction.

- Massive pair production or not in the laboratory?

- Deviations from QED or standard model? 